MARCELO KENJI HASSIMOTTO

\title{
OTIMIZAÇÃO DA PROGRAMAÇÃO DE CURTO PRAZO DE DUTO BIDIRECIONAL DE DERIVADOS DE PETRÓLEO
}


MARCELO KENJI HASSIMOTTO

\title{
OTIMIZAÇÃO DA PROGRAMAÇÃO DE CURTO PRAZO DE DUTO BIDIRECIONAL DE DERIVADOS DE PETRÓLEO
}

\author{
Dissertação apresentada à Escola \\ Politécnica da Universidade de São Paulo \\ para obtenção do título de \\ Mestre em Engenharia
}

São Paulo 


\title{
OTIMIZAÇÃO DA PROGRAMAÇÃO DE CURTO PRAZO DE DUTO BIDIRECIONAL DE DERIVADOS DE PETRÓLEO
}

\author{
Dissertação apresentada à Escola \\ Politécnica da Universidade de São Paulo \\ para obtenção do título de \\ Mestre em Engenharia \\ Área de Concentração: \\ Engenharia Química \\ Orientador: \\ Prof. Dr. José Maurício Pinto
}


Exemplar foi revisado e alterado em relação à versão original, sob responsabilidade única do autor e com anuência de seu orientador.

São Paulo, 12 de dezembro de 2007

Assinatura do autor

Assinatura do orientador

FICHA CATALOGRÁFICA

Hassimotto, Marcelo Kenji

Otimização da programação de curto prazo de duto bidirecional de derivados de petróleo / M.K. Hassimotto - ed. rev. São Paulo, 2007.

$121 \mathrm{p}$.

Dissertação (Mestrado) - Escola Politécnica da Universidade de São Paulo. Departamento de Engenharia Química.

1.Otimização matemática 2.Programação matemática 3. Programação mista 4.Transporte dutoviário 5.Dutos I.Universidade de São Paulo. Escola Politécnica. Departamento de Engenharia Química II.t. 


\section{AGRADECIMENTOS}

A minha esposa Dani, pela amizade, companheirismo e paciência nesta jornada.

Aos meus pais, pela minha formação e educação, e por entender a importância destas na minha vida.

Ao Prof. José Maurício, pela paciência, incentivos, orientações e as minuciosas revisões no trabalho. Ao Zeca e família por ter me recebido na minha estadia nos EUA.

Ao Rubens pela ajuda, paciência e todas as explicações sobre os modelos dispendidas comigo.

Aos meus amigos de Nova lorque João, Sanja, Danilo e Melissa pela amizade e ajuda na período da minha estadia nos EUA.

Aos meus colegas e amigos Vinícius, Marcel, Smania e Lincoln pela ajuda e incentivos nos trabalhos.

Aos gerentes das PETROBRAS que me permitiram a realizar este trabalho.

E a todos que contribuíram diretamente e indiretamente para realização deste trabalho. 


\section{RESUMO}

Sistemas dutoviários desempenham um papel fundamental na cadeia de suprimento da indústria de petróleo. Este tipo de sistema é responsável pelo transporte da maior parte do volume de petróleo e seus derivados. Sistemas de dutos transportam uma grande quantidade de diferentes tipos de petróleo e seus derivados a custo mais baixo que outros tipos de modais. Dutos interligam campos de produção de petróleo, portos, refinarias, centros de distribuição (ou depósitos), e mercado consumidor.

O problema estudado neste trabalho é baseado em um sistema que é composto por uma refinaria que pode transferir vários produtos para um terminal (depósito) através de um único duto. Os produtos são conjuntos de derivados de petróleo que devem ser transferidos da refinaria para o terminal ou do terminal para a refinaria. Ambos, refinaria e terminal, estão conectados a outras refinarias, terminais e mercados consumidores e com isto formam uma complexa rede de dutos. Por outro lado há um conjunto de demandas externas e internas. Esta última demanda decorre da necessidade de processamento de produtos intermediários que são misturas compostas de várias correntes intermediárias, tais como diluentes de óleos combustíveis, propano intermediário, e diesel intermediário. Com o objetivo de obter vantagens sobre a estrutura da rede de transporte, torna-se benéfica e mesmo necessária a operação do duto em ambas as direções para atender tanto à demanda externa quanto à interna.

O objetivo deste trabalho é desenvolver um modelo matemático para a programação de um sistema de poliduto. A formulação para a programação deve considerar a possibilidade de trocar o sentido do poliduto. Neste contexto, a programação de um poliduto envolve decisões tais como sentido de operação, quantidade, temporização e seqüências de produtos, com objetivo de obter uma solução ótima, considerando todas as restrições de demanda, perfil de produção, estoques e custos.

O modelo de programação é baseado em uma representação de tempo discreto e composto da área de tancagem da refinaria, um terminal, e um poliduto. Além disto o duto é dividido em segmentos de volumes iguais como em Rejowski Jr e Pinto (2003). As principais variáveis de decisão são a direção da movimentação do duto (da refinaria para terminal ou do terminal para refinaria) e o que está sendo movimentado a cada intervalo. Estas decisões são formuladas através de uma representação disjuntiva. As disjunções são transformadas em uma formulação baseada em programação matemática mista-inteira, a partir da representação Convex-hull. A função objetivo considera os custos de estocagem, movimentação e interface de produtos.

O modelo é aplicado inicialmente a um caso protótipo e posteriormente aplicado a um sistema real composto pelos terminais de São Sebastião e Guararema e o poliduto OSPLAN. Neste caso ao todo quatro famílias de produtos são transportadas: gasolina, querosene, nafta e diesel. A programação é gerada para o período de uma semana. 


\section{ABSTRACT}

Pipeline systems play a major role in the supply chain of the petroleum industry. These systems are responsible for the transportation of most of the crude oil and petroleum derivatives. Pipeline systems transfer large amounts of different petroleum types and their products at a lower cost than any other transportation mode. Pipelines interconnect oil fields, ports, refineries, distribution centers (or depots), and consumer markets.

The problem addressed is this work is based on a system that is composed by an oil refinery that must transfer multiple products through a single pipeline connected to one depot. The products are a set of petroleum derivatives that must be either transported from the refinery to the depot or from the depot to the refinery. Both depot and refinery also connect other refineries as well as other depots and customers, thus forming a complex transportation network. On the other hand, there are several demands that arise either from external customers or from refineries. The latter demand is due from the need of processing intermediate streams with components mixtures such as diluents, propane and diesel. In order to take advantage of the structure of the transportation network, it becomes beneficial and even necessary to operate the pipeline in both directions so that internal and external demands are satisfied.

The objective of this work is to develop a mathematical model for the short term scheduling of a multiproduct pipeline system. The scheduling formulation must account for the bidirectionality of the multiproduct pipeline. In this context, the scheduling a multiproduct pipeline involves the from-to decision, the product amounts, their sequence and timing, in the optimal sense, considering all constrains on demands, production rates, inventories, and costs.

The scheduling model is based on a discrete time representation and is composed by one refinery tank farm, one depot and one multiproduct pipeline. Moreover, the pipeline is divided into segments of equal volume, as in Rejowski Jr and Pinto (2003). The main decisions variables are the directions of transfer (refinery to depot or depot to refinery) and the types of products at each time interval. These decisions are formulated with a disjunctive representation. The disjunctions are represented in mixed integer formulation based on the convex-hull approach. The objective function involves inventory, transfer and product interface costs.

The model is first applied to a prototype case and after applied to a real-world system that is composed of the São Sebastião and Guararema depot and the OSPLAN pipeline. Overall four families of products are transported: gasoline, kerosene, naphtha and oil diesel. These are scheduled over a period of one week. 


\section{LISTA DE FIGURAS}

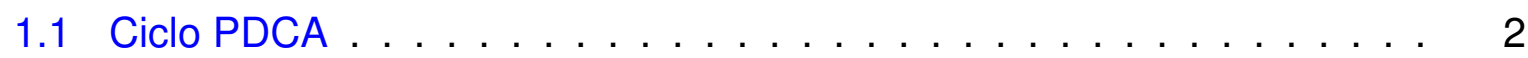

3.1 Esquema geográfico da rede de distribuição da área São Paulo . . . . 10

3.2 Representação do esquema do caso 1 de reversão do duto . . . . . . . 15

3.3 Representação do esquema do caso 2 de reversão do duto . . . . . . 16

3.4 Representação do esquema do caso 3 de reversão do duto $\ldots$. . . . 17

4.1 Representação da linha do tempo . . . . . . . . . . . . . . . 26

4.2 Representação do armazenamento na refinaria . . . . . . . . . . . . 29

4.3 Representação do armazenamento no terminal . . . . . . . . . . . . 30

4.4 Representação do duto movimentando da refinaria para o terminal . . . 31

4.5 Representação do duto movimentando do terminal para a refinaria . . . 31

5.1 Estoque da refinaria para o caso manual . . . . . . . . . . . 44

5.2 Estoque do terminal para o caso manual . . . . . . . . . . . . 44

5.3 Estoque da refinaria para o caso modelo $\ldots \ldots \ldots \ldots \ldots$

5.4 Estoque do terminal para o caso modelo . . . . . . . . . . . . 45

6.1 Ilustração da situação 1 para corte completo $\ldots \ldots \ldots$. . . . . . 56

6.2 Ilustração da situação 2 para corte completo . . . . . . . . . . . 57

6.3 Ilustração da situação 3 para corte completo . . . . . . . . . . . 57

6.4 Ilustração da situação 4 para corte completo . . . . . . . . . . . . 58

6.5 Ilustração da situação 1 para corte completo no sentido terminal-refinaria 62

6.6 Ilustração da situação 2 para corte completo no sentido terminal-refinaria 62

6.7 Ilustração da situação 3 para corte completo no sentido terminal-refinaria 63

6.8 Ilustração da situação 4 para corte completo no sentido terminal-refinaria 63 
6.9 Ilustração do exemplo $1 \ldots \ldots$. . . . . . . . . . . . . . . . . . . . 69

6.10 llustração do exemplo $2 \ldots \ldots$. . . . . . . . . . . . . . 70

7.1 Representação do armazenamento na refinaria . . . . . . . . . . . 88

7.2 Representação do armazenamento no terminal . . . . . . . . . . . 88

7.3 Estoque da refinaria para o caso manual . . . . . . . . . . . . . 94

7.4 Estoque do terminal para o caso manual . . . . . . . . . . . . . 95

7.5 Estoque da refinaria para o caso modelo $\ldots \ldots \ldots \ldots$

7.6 Estoque do terminal para o caso modelo . . . . . . . . . . . 96

7.7 Estoque da refinaria do produto $p=2$ para as 3 situações analisadas . 99

7.8 Estoque do terminal do produto $p=2$ para as 3 situações analisadas . 99

8.1 Representação do esquema do caso real de reversão do duto . . . . . 101

8.2 Estoque do produto $p=1$ na refinaria e no terminal no caso real $\ldots 110$

8.3 Estoque do produto $p=2$ na refinaria e no terminal no caso real $\ldots 110$

8.4 Estoque do produto $p=3$ na refinaria e no terminal no caso real $\ldots 111$

8.5 Estoque do produto $p=4$ na refinaria e no terminal no caso real $\ldots 111$ 


\section{LISTA DE TABELAS}

3.1 Refinarias da área São Paulo . . . . . . . . . . . . . . . . . . 11

3.2 Terminais da área São Paulo $\ldots \ldots \ldots \ldots \ldots \ldots$

3.3 Dutos de distribuição da área São Paulo . . . . . . . . . . . . . . . . 12

4.1 Procedimento de nomenclatura . . . . . . . . . . . . . . . . . 22

4.2 Índices utilizados no modelo . . . . . . . . . . . . . . . . . . 22

4.3 Parâmetros contínuos utilizados no modelo . . . . . . . . . . . . . . 23

4.4 Parâmetros binários utilizados no modelo . . . . . . . . . . . . . . . 24

4.5 Variáveis contínuas utilizadas no modelo . . . . . . . . . . . . 24

4.6 Variáveis binárias utilizadas no modelo . . . . . . . . . . . . . 25

5.1 Índices do protótipo $\mathrm{p} /$ tancagem da refinaria $\ldots \ldots \ldots$. . . . . 40

5.2 Parâmetros do protótipo $\mathrm{p} /$ tancagem da refinaria . . . . . . . . . . 40

5.3 Parâmetros do protótipo $\mathrm{p} /$ tancagem da refinaria ao longo do tempo . . 40

5.4 Parâmetros do protótipo $\mathrm{p} /$ tancagem do terminal . . . . . . . . . . 40

5.5 Parâmetros do protótipo $\mathrm{p} /$ tancagem do terminal ao longo do tempo . . 41

5.6 Parâmetros do protótipo $\mathrm{p} /$ duto . . . . . . . . . . . . . . . 41

5.7 Parâmetros do protótipo $\mathrm{p} /$ duto inicial $\ldots \ldots \ldots$. . . . . . . 41

5.8 Parâmetros do protótipo $\mathrm{p} /$ custo de estocagem e bombeamento . . . . 41

5.9 Parâmetros do protótipo $\mathrm{p} /$ custo da interface . . . . . . . . . . . . 42

5.10 Valores da função objetivo . . . . . . . . . . . . . . . . . . 42

5.11 Movimentações da refinaria para terminal $\ldots \ldots \ldots \ldots . \ldots . . .43$

5.12 Movimentações do terminal para refinaria $\ldots \ldots \ldots \ldots$. . . . . . 43

5.13 Perfis do duto no caso manual e MS . . . . . . . . . . . . . . . 43 
6.1 Parâmetros associados ao corte simples . . . . . . . . . . . . 48

6.2 Parâmetros associados ao corte simples por instante de tempo . . . . 51

6.3 Parâmetros associados ao corte completo . . . . . . . . . . . . . . 54

6.4 Parâmetros associadas aos cortes completos por tempo . . . . . . . . 74

6.5 Comparação do tempo computacional . . . . . . . . . . . . . . 83

6.6 Estatística dos modelos . . . . . . . . . . . . . . . . . . . . 83

7.1 Variáveis associadas a reformulação . . . . . . . . . . . . 86

7.2 Parâmetros associados à reformulação $\ldots \ldots \ldots \ldots$. . . . . 87

7.3 Parâmetros do protótipo $\mathrm{p} /$ faixa da tancagem . . . . . . . . . . 91

7.4 Parâmetros do protótipo $\mathrm{p} /$ penalização fora da faixa . . . . . . . . . 91

7.5 Comparação do tempo computacional para reformulação da função ob-

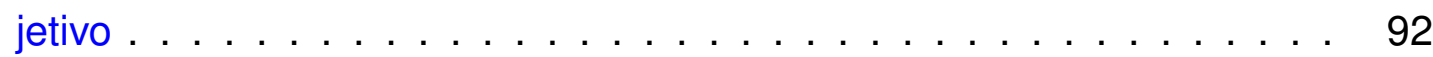

7.6 Valores da função objetivo . . . . . . . . . . . . . . . . . 92

7.7 Movimentações da refinaria para terminal . . . . . . . . . 93

7.8 Movimentações do terminal para refinaria $\ldots \ldots \ldots \ldots . \ldots 93$

7.9 Perfis do duto no caso manual e MPE . . . . . . . . . . . . 93

7.10 Movimentações da refinaria para terminal $\ldots \ldots \ldots \ldots$. . . . 98

7.11 Movimentações do terminal para refinaria $\ldots \ldots \ldots \ldots . \ldots 98$

8.1 Índices do caso real . . . . . . . . . . . . . . . . . . . . . 102

8.2 Parâmetros do caso real para tancagem da refinaria . . . . . . . . . 102

8.3 Parâmetros do caso real para tancagem da refinaria ao longo do tempo 102

8.4 Parâmetros do caso real para tancagem do terminal . . . . . . . . . 103

8.5 Parâmetros do caso real para tancagem do terminal ao longo do tempo 103

8.6 Parâmetros do caso real para a faixa desejável de estoques . . . . . . . 104

8.7 Parâmetros para condição inicial do duto . . . . . . . . . . . . . . . 104

8.8 Parâmetros do caso real para o custo de bombeamento . . . . . . . . . 104 
8.9 Parâmetros do caso real para o custo da interface . . . . . . . . . . . . 104

8.10 Parâmetros do caso real para penalização de estoques . . . . . . . . . 105

8.11 Valores da função objetivo para caso real . . . . . . . . . . . . . . . . . 105

8.12 Valores da função objetivo para caso real . . . . . . . . . . . . . . 106

8.13 Movimentações da refinaria para o terminal e vice-versa para o caso real 106

8.14 Perfis do duto no caso real e MPE . . . . . . . . . . . . . . . . . . 108 


\section{LISTA DE SIGLAS}

CLP Constraint Logic Programming, em português Programação por Restrições

GAMS General Algebraic Modeling System

GLP Gás Liquefeito do Petróleo

IA Inteligência Artificial

LP $\quad$ Linear Programming, em português Programação Linear

MILP Mixed-Integer Linear Programming, em português Programação

Matemática Mista-Inteira Linear

MINLP Mixed-Integer NonLinear Programming, em português Programação Matemática Mista-Inteira Não Linear

PETROBRAS Petróleo Brasileiro S.A.

PDCA Plan-Do-Check-Act, em português Planeja-Executa-Verifica-Corrige 


\section{SUMÁRIO}

1 Introdução 1

1.1 Motivação . . . . . . . . . . . . . . . . . . . . . . . . 1

1.2 Objetivo e estrutura $\ldots \ldots \ldots \ldots \ldots$

2 Revisão da Literatura $\quad 5$

2.1 Planejamento e programação na indústria de petróleo . . . . . . . . . . 5

2.2 Transporte dutoviário . . . . . . . . . . . . . . . . . 7

3 Descrição do Problema 9

3.1 Introdução . . . . . . . . . . . . . . . . . . . . 9

3.2 Descrição da rede de dutos de distribuição da área São Paulo . . . . . 9

3.3 Problema de programação operacional dos dutos . . . . . . . . . . . . . . . . . . 13

3.4 Abordagem de programação operacional dos dutos com reversão . . . 14

4 Modelagem do Problema de Programação Operacional dos Dutos 18

4.1 Introdução . . . . . . . . . . . . . . . . . . . . 18

4.2 Considerações gerais . . . . . . . . . . . . . . . . . . . . 18

4.3 Hipóteses do modelo . . . . . . . . . . . . . . . . . . . . . . . . . . . . . 19

4.4 Estrutura do modelo . . . . . . . . . . . . . . . . . . . . . 21

4.4 Notação . . . . . . . . . . . . . . . . . . . . . 21

4.4 .2 Representação do tempo . . . . . . . . . . . . . . . . 26

4.4.3 Modelos dos equipamentos . . . . . . . . . . . . . . . . 26 
4.4.3.1 Armazenamento . . . . . . . . . . . . . . . 27

4.4.3.2 Dutos . . . . . . . . . . . . . . . . . 30

4.4.4 Modelagem das restrições de negócio . . . . . . . . . . . 35

4.4 .5 Função Objetivo . . . . . . . . . . . . . . . . . 37

4.5 Resumo do modelo . . . . . . . . . . . . . . . . . . . 38

5 Resultados $\quad 39$

5.1 Métodos de solução . . . . . . . . . . . . . . . . . . . . . . . . . 39

5.2 Exemplo Utilizado . . . . . . . . . . . . . . . . . . . . . . . . 39

5.2.1 Sistema Protótipo . . . . . . . . . . . . . . . . . . . . 39

5.2 .2 Resultados . . . . . . . . . . . . . . . . . . . . . 42

5.2 .3 Discussão dos resultados . . . . . . . . . . . . . . . . . . . 46

6 Desenvolvimento de Restrições de Corte para o modelo MILP 47

6.1 Introdução . . . . . . . . . . . . . . . . . . . 47

6.2 Corte Simples . . . . . . . . . . . . . . . . . . . . 47

6.2.1 Descrição de Corte Simples . . . . . . . . . . . . . . . . . . 47

6.2.2 Modelagem de Corte Simples . . . . . . . . . . . . . . . . . 48

6.2.2.1 Restrições de corte simples por instante de tempo . . . 51

6.3 Corte Completo . . . . . . . . . . . . . . . . . . . . . . . 53

6.3.1 Descrição de Corte Completo . . . . . . . . . . . . . . . . 53

6.3.2 Modelagem de Corte Completo . . . . . . . . . . . . . . . . 54

6.3.2.1 Exemplos ilustrativos dos cortes válidos . . . . . . . . 68

6.3.2.2 Restrições de corte por instante de tempo . . . . . . 74

6.4 Resultados . . . . . . . . . . . . . . . . 82

7 Reformulação do Custo de Estocagem no Modelo MILP 85

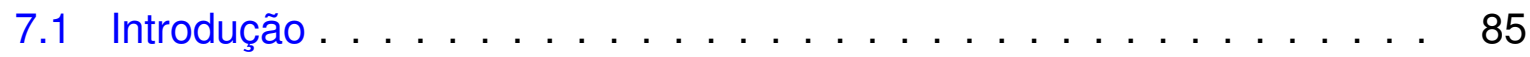


7.2 Descrição . . . . . . . . . . . . . . . . . . . 85

7.3 Modelagem . . . . . . . . . . . . . . . 86

7.4 Resultados Computacionais dos Modelos . . . . . . . . . . . . . . 91

7.5 Análise de Sensibilidade do Parâmetro de Penalização . . . . . . . . 97

8 Programação de dois terminais interligados 100

8.1 Introdução . . . . . . . . . . . . . . . . . . . . . 100

8.2 Descrição do Caso Real . . . . . . . . . . . . . . . . . . . . . . . . 100

8.3 Dados utilizados . . . . . . . . . . . . . . . . . . . . . . . . . . 101

8.4 Resultados Computacionais . . . . . . . . . . . . . . . . . . . . . . . 105

8.5 Discussão dos Resultados . . . . . . . . . . . . . . . . . . . . . . 112

9 Conclusões e propostas de continuação do trabalho 113

9.1 Principais Contribuições do Trabalho . . . . . . . . . . . . . . . . . 113

9.2 Sumário da dissertação . . . . . . . . . . . . . . . . . . . . . . . . 114

9.3 Propostas de continuação do trabalho . . . . . . . . . . . . . . 115 


\section{INTRODUÇÃO}

\subsection{MOTIVAÇÃO}

A abertura do mercado brasileiro de petróleo, pela Emenda Constitucional $n^{\circ} 9$ de 1995 e Lei Ordinária n9.478 de 06/08/1997, gerou na Petrobras (Petróleo Brasileiro S.A.) a necessidade de se adaptar a uma nova realidade. Nesta nova realidade a busca de soluções rentáveis em termos econômicos tornou-se uma premissa básica, e não mais a meta de abastecer o país com o mínimo custo, como era a prática anterior à abertura de mercado.

O transporte de petróleo e seus derivados pode ser efetuado de várias maneiras. As opções mais comuns são: modal dutoviário, modal rodoviário, modal ferroviário e modal aquaviário. Entretanto a opção modal dutoviário é a mais adequada para a indústria petrolífera na maioria dos casos, devido à possibilidade de transportar grandes volumes de forma contínua (ASSIS, 2002). Com isto seus custos são menores, e outros fatores que corroboram o seu uso são os fatos de ser mais confiável (REJOWSKI $J R, 2001$ ) e mais ambientalmente seguro (ASSIS, 2002).

No caso de empresas petrolíferas integradas, as operações de todas as unidades dos segmentos de produção de petróleo (poços e plataformas), transporte (navios, dutos), refino (refinarias) e distribuição (terminais) são efetuadas em conjunto. O modal dutoviário, uma vez instalado, é também bastante interessante porque facilita a movimentação de diversos produtos entre estas unidades, flexibilizando o gerenciamento de operações pela empresa, e com isto oferecendo maiores oportunidades de negócio.

Neste quadro de flexibilidade e oportunidades, a cultura adotada de qualidade total e melhoria contínua remete à metodologia como o ciclo PDCA (Plan-Do-Check-Act), tanto na indústria de forma geral, como também na petrolífera. Este ciclo é dividido em quatro etapas: planejar, executar, verificar (avaliar), agir (corrigir), como mostrado 
na figura 1.1. Ao fim do ciclo as correções serão incorporadas ao novo planejamento.

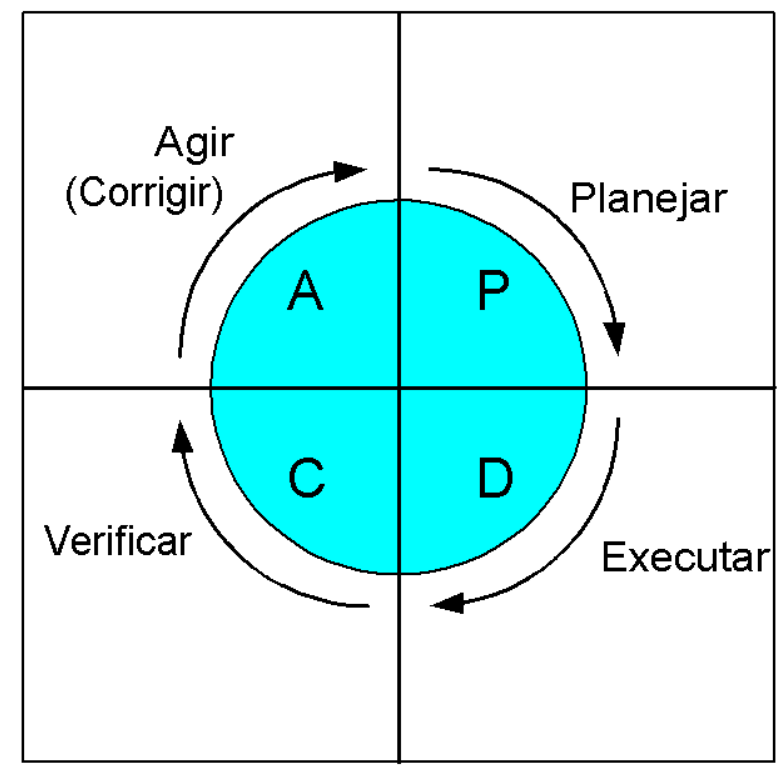

Figura 1.1: Ciclo PDCA

Neste cenário pode-se inferir que a indústria petrolífera também trabalha de forma similar, realizando o planejamento operacional, a execução das atividades, as avaliações destas atividades e as devidas correções para um novo planejamento. As atividades relacionadas ao planejamento, tais como compra de matéria-prima, transporte e armazenamento de petróleo e seus derivados, processamento de insumos e produção de produtos finais, podem ser divididas em camadas ou níveis de hierarquia.

Segundo Magalhães (2004), há uma hierarquia na tomada de decisão em nível de planejamento operacional para o refino. De forma similar pode-se citar os níveis hierárquicos também para o planejamento tático de dutos, e são os seguintes:

\section{- Planejamento Corporativo}

Usualmente é o planejamento das atividades da corporação para um horizonte de diversos meses. Normalmente a ferramenta de auxílio a tomada de decisão é um modelo matemático de programação linear (LP), no qual estão incorporados recursos de transporte, de armazenamento, de processamento, demandas de produtos finais e intermediários, produção de matérias-primas, compra de matérias-primas, produção de produtos finais, entre outros. A maioria das informações é utilizada na forma agregada.

\section{- Planejamento Local}

Assim como o planejamento corporativo, normalmente a ferramenta de auxílio à 
tomada de decisão é baseada em um modelo LP. Entretanto o período de planejamento é menor em relação ao corporativo, em torno de um a dois meses, e o escopo do modelo são as decisões de movimentações entre refinarias, terminais (depósitos) e consumidores. Assim como no caso do planejamento corporativo, a maioria das informações é empregada na forma agregada.

\section{- Programação Local ou Scheduling ${ }^{1}$}

Diferentemente dos níveis de planejamento, normalmente a ferramenta de auxílio à tomada de decisão é um simulador (verificador). O período da programação local é menor em relação ao planejamento local. Outra diferença em relação aos níveis de planejamento é o nível de agregação das informações, pois normalmente as informações de tempo e estocagem estão na forma desagregada.

\subsection{OBJETIVO E ESTRUTURA}

Este trabalho é motivado em parte pelo problema da programação operacional dos dutos, realizado pela Gerência de Operações SP da área de Abastecimento da PETROBRAS.

Como observado no item anterior (1.1), dentro da etapa de planejamento há vários níveis hierárquicos. No entanto nos níveis de planejamento corporativo e planejamento local já existem ferramentas que utilizam técnicas de otimização para auxílio à decisão consagradas (LP), enquanto no nível da programação local esta ferramenta de otimização não se encontra disponível.

A necessidade de soluções rentáveis, a falta de uma ferramenta de otimização consagrada para o problema, e a flexibilidade de movimentações de produtos entre as unidades da empresa motivam o desenvolvimento de uma ferramenta similar às utilizadas para o planejamento (corporativo e local), que gere soluções ótimas em relação ao modelo para o problema de planejamento local.

O objetivo deste trabalho é desenvolver uma formulação que contribua para que neste nível hierárquico de tomada de decisão haja uma ferramenta que possa auxiliar, gerando respostas ótimas em relação ao problema. Porque como mencionado acima as ferramentas consagradas para este nível são somente do tipo simulação, que fazem a função de verificador, validando ou não as soluções geradas pelo próprio

\footnotetext{
${ }^{1} \mathrm{O}$ termo scheduling tem o mesmo significado de programação.
} 
programador $^{2}$.

Este trabalho está estruturado da seguinte forma: o capítulo 2 apresenta a revisão da literatura; nos capítulos 3 e 4 são descritos respectivamente o problema de operação dutoviária e o modelo matemático correspondente; os resultados são mostrados e discutidos no capítulo 5. No capítulo 6 são apresentados os cortes desenvolvidos no modelo MILP e seus resultados. No capítulo 7 é apresentada uma reformulação da função objetivo para tornar-se mais aderente à forma de operação. No capítulo 8 é apresentado um caso do problema real que é descrito no capítulo 3. Finalmente, o capítulo 9 apresenta as principais conclusões deste trabalho, bem como as propostas de continuação do mesmo.

\footnotetext{
${ }^{2}$ programador executa a tarefa de programação local.
} 


\section{REVISÃO DA LITERATURA}

Neste capítulo são apresentadas e discutidas as principais contribuições nas áreas de planejamento e programação na indústria de petróleo (item 2.1) e no transporte dutoviário em particular (item 2.2).

\subsection{PLANEJAMENTO E PROGRAMAÇÃO NA INDÚSTRIA DE PETRÓLEO}

Segundo Lee et al. (1996), o planejamento corporativo na indústria petrolífera já é bastante difundido, sendo utilizado há bastante tempo, e atualmente ferramentas computacionais encontram-se em desenvolvimento para a programação local ou simplesmente programação de produção.

Como mencionado no item 1.1, a técnica mais popular presente nas ferramentas comerciais para abordar o problema de programação local é a simulação (MAGALHÃES et al., 1998). Segundo Magalhães (2004), a principal função das ferramentas de simulação é retornar as conseqüências das programações (atividades) propostas para a planta modelada, validando e verificando as possíveis inviabilidades, como por exemplo sobrealocação de recursos ou falta de produtos. Este tipo de abordagem é limitada na busca de soluções automáticas, isto é, não fornece como resposta automática uma solução do problema, e portanto a busca de uma solução fica restrita ao ciclo da tentativa-e-erro.

Dentre as técnicas de solução de problemas de scheduling, segundo Magalhães (2004), são utilizadas aquelas baseadas em otimização ou programação matemática. Estas são normalmente das classes de Programação Matemática Mista-Inteira Linear (MILP) ou de Programação Matemática Mista-Inteira Não Linear (MINLP). As variáveis inteiras do modelo representam algumas decisões de natureza discretas que devem ser tomadas para a solução do problema, como por exemplo seqüência de en- 
vio/recebimento de petróleos e/ou derivados, processamento de diferentes tipos de petróleo, seleção de um determinado equipamento; nota-se que tais decisões podem ser função do tempo. Quanto à linearidade ou não do problema, esta está mais relacionada à necessidade de representação mais rigorosa ou não das propriedades/atributos físicos e/ou químicos dos recursos envolvidos, como exemplo propriedades de produtos (misturas), perda de carga ao longo do duto.

Dentre outras técnicas de solução, existem as heurísticas e as meta-heurísticas. A busca de solução heurística normalmente se baseia em um sistema especialista, o qual consiste no modelamento matemático de regras elaboradas por especialistas, normalmente do tipo se-então. Este tipo de abordagem é importante para preservar o conhecimento do especialista, mas representa um pensamento particular na busca de uma solução, ficando obsoleto caso sejam alteradas as situações usuais e conhecidas. Dentre as técnicas meta-heurísticas, existem o algoritmo genético, lógica difusa (fuzzy), a rede neural, entre outras.

Para o problema relacionado ao planejamento local podemos citar que para a área de produção de diesel dentro de uma refinaria, Moro et al. (1998) desenvolveram um modelo utilizando a técnica MINLP, onde as não linearidades do modelo advêm dos cálculos de mistura das propriedades. A representação para otimização da cadeia de suprimento em múltiplos períodos com incertezas de demandas e preços de produtos foram apresentadas por Escudero et al. (1999), onde as incertezas são tratadas com uma formulação estocástica. Neiro e Pinto (2004) desenvolveram um modelo não linear (propriedades de produtos) para o gerenciamento da cadeia de suprimento de petróleo com múltiplos períodos, gerando um MINLP de grande porte. Modelos que representam gerenciamento de riscos financeiros para planejamento de uma refinaria foram desenvolvidos por Pongsakdi et al. (2006).

Para o problema relacionado à programação local vários trabalhos foram desenvolvidos utilizando a técnica de programação matemática. Em virtude das dimensões dos problemas decisórios, as abordagens são direcionadas para sub-sistemas da indústrias petrolífera. Shah (1996) desenvolveu um modelo para área de petróleo (desde o desembarque do óleo até o processamento) no qual foi utilizada a representação do tempo discreto. Para produção de GLP de uma refinaria (tancagem de produtos intermediários, unidades de processo e tancagem de produtos finais), Pinto e Moro (2000) desenvolveram um modelo utilizando a representação em tempo contínuo. GötheLundgren et al. (2002) desenvolveram um modelo para planejamento e programação 
de unidades de processo, com a representação de tempo discreta. Para produção de produtos pesados (asfaltos e óleos combustíveis) dentro da refinaria, Joly e Pinto (2003) desenvolveram dois modelos (MINLP e MILP), ambos com representação do tempo discreto. Para recebimento petróleo e seleção de petróleo para carga de unidades de processamento dentro de uma refinaria, Moro e Pinto (2004) desenvolveram com representação do tempo contínuo e com não linearidades (MINLP). Magalhães (2004) desenvolveu uma formulação com a representação do tempo contínuo, onde a abrangência é um terminal, um duto de petróleo e parte de uma refinaria. Esta formulação (MINLP) baseou-se em uma decomposição, interagindo três modelos matemáticos (MILP). Para uma planta de produção de óleo lubrificante e parafinas, Casas-Liza e Pinto (2005) desenvolveram dois modelos, um com representação do tempo discreta e outra com tempo contínuo. Pinto et al. (2000) sintetizaram as informações de trabalhos relacionados ao planejamento local e a programação de produção, citando aplicações de simulação para refinarias, e modelos matemáticos para subsistemas de uma refinaria.

\subsection{TRANSPORTE DUTOVIÁRIO}

Para o planejamento local e programação específicos de dutos foram desenvolvidas formulações envolvendo diversos aspectos. Hane e Ratliff (1995) desenvolveram um modelo onde o foco é na otimização da programação do seqüenciamento de bombeamento de produtos, isto é, um item importante é a interface de produtos, contabilizado na função de custo. Utilizando uma formulação de inteligência artificial (IA) que privilegia a determinação de uma solução viável, ao invés de uma solução ótima, Sasikumar et al. (1997) desenvolveram uma formulação mista utilizando um sistema especialista mais uma função matemática que avalia a solução. Para o planejamento de uma malha de dutos, refinarias e terminais, Assis (2002) modelou com representação de múltiplos períodos. Na programação do transporte de petróleo, utilizando recursos de portos, dutos e tancagem (terminais e refinaria), Más e Pinto (2003) desenvolveram uma formulação MILP, representando o tempo de forma contínua e decompondo o problema em modelos do porto e tancagem, integrando os modelos através de uma representação dos dutos. Rejowski Jr e Pinto (2003) desenvolveram para a programação da tancagem de uma refinaria, um poliduto e vários terminais que efetuavam sangria ${ }^{1}$, um modelo com representação do tempo discreto e considerando as inter-

\footnotetext{
${ }^{1}$ retirada de produto ao longo do duto
} 
faces de produtos. A formulação baseou-se na programação disjuntiva. Rejowski Jr e Pinto (2004) apresentaram uma nova formulação, o qual continha na formulação cortes no espaço de soluções (restrições que eliminam parte da região viável do problema relaxado), melhorando o desempenho computacional. Para o escopo de uma refinaria, um poliduto e um terminal, Magatão et al. (2004) desenvolveram um algoritmo para solucionar o problema, no qual foi decomposto em um modelo CLP-MILP híbrido. Com o escopo semelhante ao apresentado por Rejowski Jr e Pinto (2003), Cafaro e Cerdá (2004) apresentaram uma formulação de tempo contínuo em um modelo MILP. 


\section{DESCRIÇÃO DO PROBLEMA}

\subsection{INTRODUÇÃO}

Este capítulo descreve os aspectos importantes de um sistema de transporte e armazenamento de derivados de petróleo. O problema abordado neste trabalho corresponde a um sub-sistema deste sistema de transporte aborda a programação operacional ou scheduling das transferências dos produtos derivados do petróleo entre refinarias e terminais.

No item 3.2 são descritos os principais componentes do sistema que é utilizado como base para este trabalho, a saber aquele da região São Paulo, que contém refinarias, terminais, distribuidores, estações de bombeamentos e dutos. No item 3.3 são descritas as atividades relacionadas à função de programação de dutos que são diretamente correspondente ao sub-sistema estudado nesta dissertação e no item 3.4 é discutida a abordagem com relação à reversão do sentido da operação dos dutos.

\subsection{DESCRIÇÃO DA REDE DE DUTOS DE DISTRIBUI- ÇÃO DA ÁREA SÃO PAULO}

A rede de dutos de distribuição de derivados de petróleo interliga refinarias, terminais, distribuidores e grandes consumidores de derivados.

Os dados descritos sobre a rede de distribuição da área São Paulo foram obtidos a partir do portal na internet da Petrobras S.A. e da Transpetro S.A.; esta última é a empresa responsável pela movimentação do petróleo e seus derivados. As informações obtidas são as seguintes:

- Refinarias e suas capacidades de refino da área São Paulo;

- Terminais e suas capacidades de armazenamento da área São Paulo; 
- Estações de bombeamento e suas capacidades da área São Paulo;

- Dutos de interligação e suas capacidades da área São Paulo.

A figura 3.1 representa esquematicamente os principais pontos de produção, distribuição e consumo e suas interligações, ou seja representa a distribuição geográfica das refinarias, terminais, dutos, estações de bombeamento.

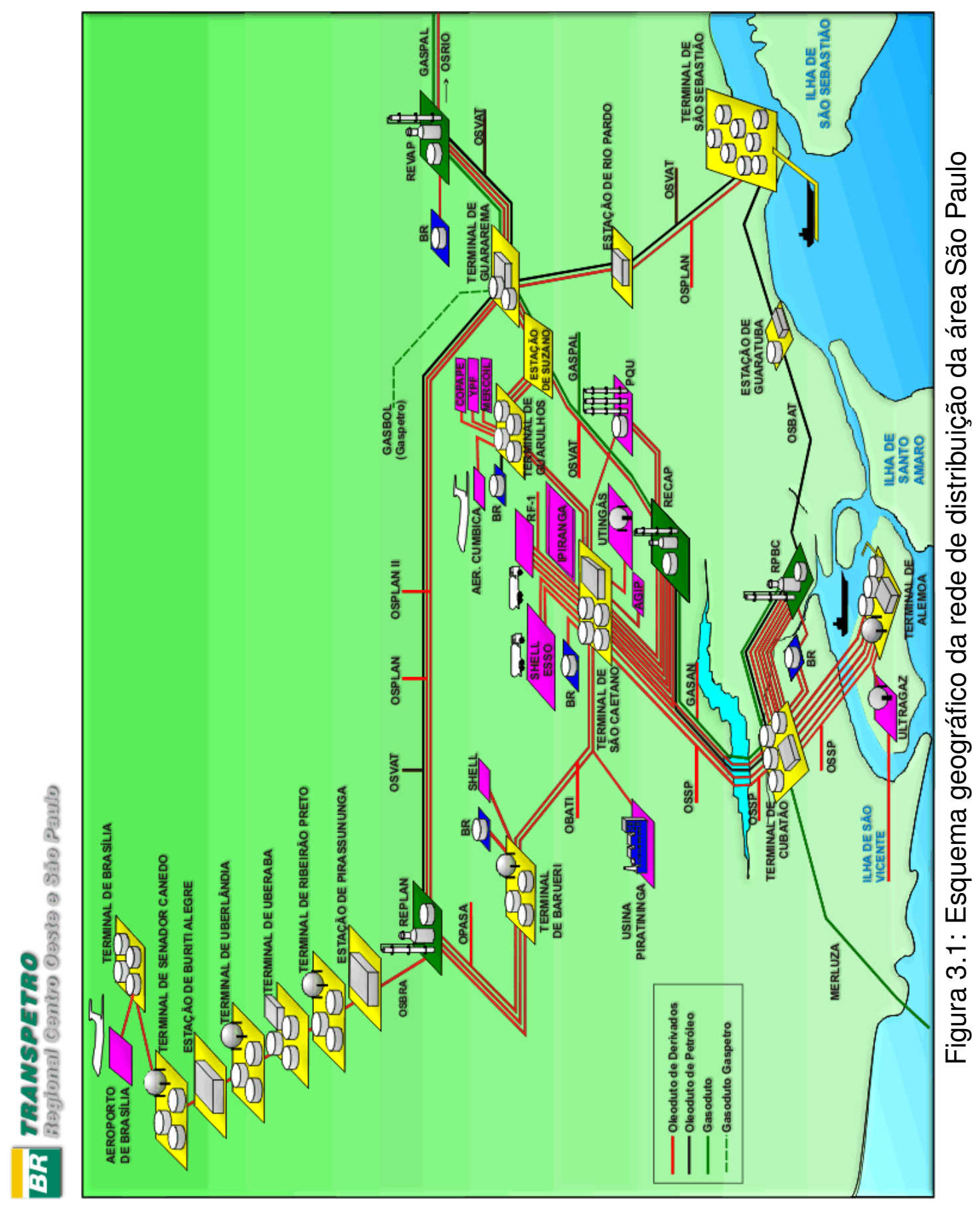


As refinarias são apresentadas na Tabela 3.1, bem como suas capacidades de processamento de óleo cru.

Tabela 3.1: Refinarias da área São Paulo

\begin{tabular}{|l|l|l|l|}
\hline \multicolumn{1}{|c|}{ Refinaria } & \multicolumn{1}{c|}{ Localização } & \multicolumn{2}{c|}{$\begin{array}{c}\text { Capacidade de } \\
\text { Processamento }\end{array}$} \\
\hline Planalto Paulista - REPLAN & Paulínia & $360.000 \mathrm{bpd}$ & $57.240 \mathrm{~m}^{3} / \mathrm{d}$ \\
\hline $\begin{array}{l}\text { Presidente Bernardes - } \\
\text { RPBC }\end{array}$ & Cubatão & $170.000 \mathrm{bpd}$ & $27.000 \mathrm{~m}^{3} / \mathrm{d}$ \\
\hline Capuava - RECAP & Mauá & $53.000 \mathrm{bpd}$ & $8.430 \mathrm{~m}^{3} / \mathrm{d}$ \\
\hline Henrique Laje - REVAP & S. J. dos Campos & $226.000 \mathrm{bpd}$ & $36.000 \mathrm{~m}^{3} / \mathrm{d}$ \\
\hline
\end{tabular}

Os terminais são apresentados na Tabela 3.2, bem como suas capacidades de armazenamento.

Tabela 3.2: Terminais da área São Paulo

\begin{tabular}{|c|c|c|}
\hline Terminal & Localização & $\begin{array}{c}\text { Capacidade de } \\
\text { Armazenamento }\end{array}$ \\
\hline Barueri & Barueri & $193.146 \mathrm{~m}^{3}$ \\
\hline Cubatão & Cubatão & $164.552 \mathrm{~m}^{3}$ \\
\hline Guararema & Guararema & $927.333 \mathrm{~m}^{3}$ \\
\hline Guarulhos & Guarulhos & $165.200 \mathrm{~m}^{3}$ \\
\hline Santos & Santos & $336.425 \mathrm{~m}^{3}$ \\
\hline São Caetano & São Caetano do Sul & $196.859 \mathrm{~m}^{3}$ \\
\hline São Sebastião & São Sebastião & $1.823 .111 \mathrm{~m}^{3}$ \\
\hline
\end{tabular}

No total existem três estações de bombeamento localizadas em Guaratuba, Rio Pardo e Suzano. Estas estações não possuem área de armazenagem, e a sua função principal é aumentar a capacidade de vazão dos dutos.

Os dutos de distribuição são apresentados na Tabela 3.3, bem como os diâmetros nominais e os comprimentos, os quais determinam os volumes de armazenamento entre as extremidades. 
Tabela 3.3: Dutos de distribuição da área São Paulo

\begin{tabular}{|c|c|c|c|c|}
\hline Duto & Extremidade 1 & Extremidade 2 & $\begin{array}{l}\text { Diâmetro } \\
\text { (in) }\end{array}$ & $\begin{array}{l}\text { Comprimento } \\
(\mathrm{km})\end{array}$ \\
\hline OSSP-PE & Cubatão & RECAP & 12 & 24 \\
\hline OSSP-LA & Cubatão & RECAP & 14 & 24 \\
\hline OSSP-LB & Cubatão & S. Caetano do Sul & 10 & 37 \\
\hline OSSP-LC & Cubatão & S. Caetano do Sul & 18 & 37 \\
\hline OSSP-OC & Cubatão & S. Caetano do Sul & 18 & 37 \\
\hline GASAN & Santos & RECAP & 12 & 36 \\
\hline OPASA & Barueri & REPLAN & 14 & 97 \\
\hline OPASA & Barueri & REPLAN & 10 & 97 \\
\hline OPASA & Barueri & REPLAN & 16 & 97 \\
\hline OSRIO & Guararema & Lorena & 16 & 133 \\
\hline OSVAT & Guararema & REVAP & 16 & 38 \\
\hline OSVAT & Guararema & REVAP & 12 & 38 \\
\hline OSVAT & Guararema & REVAP & 24 & 38 \\
\hline OSVAT & Guararema & REVAP & 34 & 38 \\
\hline OSVAT & Guararema & REPLAN & 30 & 153 \\
\hline OSVAT & Guararema & Guarulhos & 22 & 59 \\
\hline OSVAT & Guararema & Guarulhos & 24 & 59 \\
\hline OSVAT & Guararema & Rio Pardo & 38 & 49 \\
\hline OSVAT-II & Guararema & Suzano & 16 & 34 \\
\hline OSPLAN & Guararema & Rio Pardo & 24 & 49 \\
\hline OSPLAN & Guararema & REPLAN & 24 & 153 \\
\hline GASPAL & Lorena & Suzano & 22 & 167 \\
\hline GASPAL & Lorena & Suzano & 22 & 34 \\
\hline OGUAER & Guarulhos & Cumbica & 6 & 7 \\
\hline OGUAER & Guarulhos & Cumbica & 10 & 7 \\
\hline GASPAL & Suzano & RECAP & 22 & 28 \\
\hline SR-16 & Suzano & RECAP & 16 & 29 \\
\hline UG-22 & Guarulhos & S. Caetano do Sul & 22 & 25 \\
\hline UGESC & Guarulhos & S. Caetano do Sul & 24 & 25 \\
\hline GG-22 & Guarulhos & Guararema & 22 & 58 \\
\hline ZGR & Guarulhos & Suzano & 16 & 25 \\
\hline RVESC & Guarulhos & REVAP & 24 & 95 \\
\hline OSSP-ES A8 & Santos & Cubatão & 18 & 12 \\
\hline
\end{tabular}




\begin{tabular}{|c|c|c|c|c|}
\hline Duto & Extremidade 1 & Extremidade 2 & $\begin{array}{c}\text { Diâmetro } \\
\text { (in) }\end{array}$ & $\begin{array}{c}\text { Comprimento } \\
(\mathrm{km})\end{array}$ \\
\hline OSSP-CL A12 & Santos & Cubatão & 14 & 12 \\
\hline OSSP-CL A4 & Santos & Cubatão & 18 & 12 \\
\hline OSSP-CL A6 & Santos & Cubatão & 14 & 12 \\
\hline OSSP-CL A9 & Santos & Cubatão & 10 & 12 \\
\hline OSSP-RC08 & S. Caetano do Sul & RECAP & 8 & 10 \\
\hline OSSP-RC10 & S. Caetano do Sul & RECAP & 10 & 10 \\
\hline OSSP-RC16 & S. Caetano do Sul & RECAP & 16 & 10 \\
\hline OSSP-SPA2 & S. Caetano do Sul & RECAP & 14 & 10 \\
\hline OSVAT-RC16 & S. Caetano do Sul & Petroquímica União & 16 & 8,5 \\
\hline OBATI-CL & S. Caetano do Sul & Barueri & 14 & 50 \\
\hline OBATI-ES & S. Caetano do Sul & Barueri & 14 & 50 \\
\hline OSBAT & São Sebastião & Cubatão & 24 & 123 \\
\hline OSPLAN & São Sebastião & REPLAN & 24 & 237 \\
\hline OSVAT & São Sebastião & REVAP & $42 / 38$ & 84 \\
\hline
\end{tabular}

\subsection{PROBLEMA DE PROGRAMAÇÃO OPERACIONAL DOS DUTOS}

O problema de programação operacional dos dutos para o programador, sob uma visão simplificada, é atender os terminais com produtos que são fornecidos pelas refinarias em um curto espaço de tempo, isto é, em torno de uma semana. Neste contexto do problema da programação operacional dos dutos, os terminais têm como função básica armazenar produtos e abastecer as distribuidoras e os grandes consumidores, as refinarias têm como função básica produzir produtos acabados para atender os terminais e produzir produtos intermediários ou finais para outras refinarias.

O detalhamento da programação operacional dos dutos em uma linguagem simplificada consiste em determinar o seqüenciamento dos bombeamentos (quantidades de um determinado produto) a serem efetuados pelas refinarias e terminais a fim de satisfazer as demandas dos produtos e não violar as restrições operacionais e de negócios, tais como: vazão mínima e máxima de bombeamento, estoque mínimo e máximo de produtos nas refinarias e terminais, seqüenciamento de bombeamentos de produtos (evitando contaminação entre os derivados adjacentes), etc.

O problema estudado por Rejowski Jr (2001), o qual descreve a distribuição duto- 
viária de derivados de petróleo, faz referência a uma refinaria com um duto e vários terminais; sua estrutura é de certo modo semelhante à do problema que está sendo abordado. Assis (2002) descreve o problema da distribuição dutoviária de derivados de petróleo da área do Estado de São Paulo, também apresenta alguma semelhança com o sistema estudado neste trabalho, com a diferença em relação à representação do domínio do tempo, no qual Assis (2002) o representa de forma agregada.

Devido a possibilidade de transportar vários produtos através de um duto, o seqüenciamento de bombeamentos está entre as atividades ligadas ao problema de programação operacional de dutos. O seqüenciamento de bombeamentos determina uma condição de contato de diferentes produtos dentro do duto. Este contato entre produtos diferentes gera uma região (volume dentro do duto) em que há uma mistura dos produtos, é denominada interface, de acordo com Joly (1999), Assis (2002) e Rejowski Jr e Pinto (2003). A existência da interface no transporte acarreta em contaminação em ambos os produtos em contato e por conseqüência perdas. Há regras de negócios que ou evitam ou impedem que certas seqüências de bombeamento sejam efetuadas, isto é ou é permitida a interface mas com restrições ou não é permitida a interface entre alguns produtos. Uma causa para o impedimento das interfaces entre certos produtos é principalmente devido ao fato que na contaminação dos produtos, os mesmos ou um deles ficará fora de especificação.

Além do seqüenciamento de bombeamentos, outra atividade ligada ao problema de programação operacional está em determinar quando não há necessidade de movimentar produtos através do duto. Quando ocorre esta situação é necessário verificar qual produto ficará preenchendo o duto, porque certos tipo de produtos não podem ficar parados dentro do duto, pois a longa permanência dos mesmos dentro do duto pode causar danos a este. Um exemplo são alguns tipos de óleo combustíveis cuja longa permanência dentro do duto pode causar sua obstrução pelo congelamento do produto.

\subsection{ABORDAGEM DE PROGRAMAÇÃO OPERACIONAL DOS DUTOS COM REVERSÃO}

Assis (2002) e Rejowski Jr e Pinto (2003) consideraram fluxo unidirecional de produtos, devido às características dos problemas estudados pelos autores. Porém, neste caso, deve-se considerar a possibilidade de reversão da operação dos dutos, isto é 
que os dutos podem reverter o fluxo de produtos, possibilitando em quaisquer extremidades do duto enviarem ou receberem produtos.

A operação de reversão de dutos é utilizada em algumas situações. Uma das situações é: existem a extremidade "A" e a extremidade "B" do duto, e somente há possibilidade de transferir produtos entre as extremidades através do duto, e há necessidade de transportar produtos da extremidade "A" para a extremidade "B", e também transportar outros produtos da extremidade "B" para a extremidade "A". Por exemplo, no passado recente a REPLAN necessitava de um determinado componente para produção de diesel e a REVAP produzia este componente; por outro lado, a REPLAN produzia um determinado componente que a REVAP necessitava para fazer a composição da produção de gasolina. A figura 3.2 representa a situação descrita acima através de um esquema gráfico.

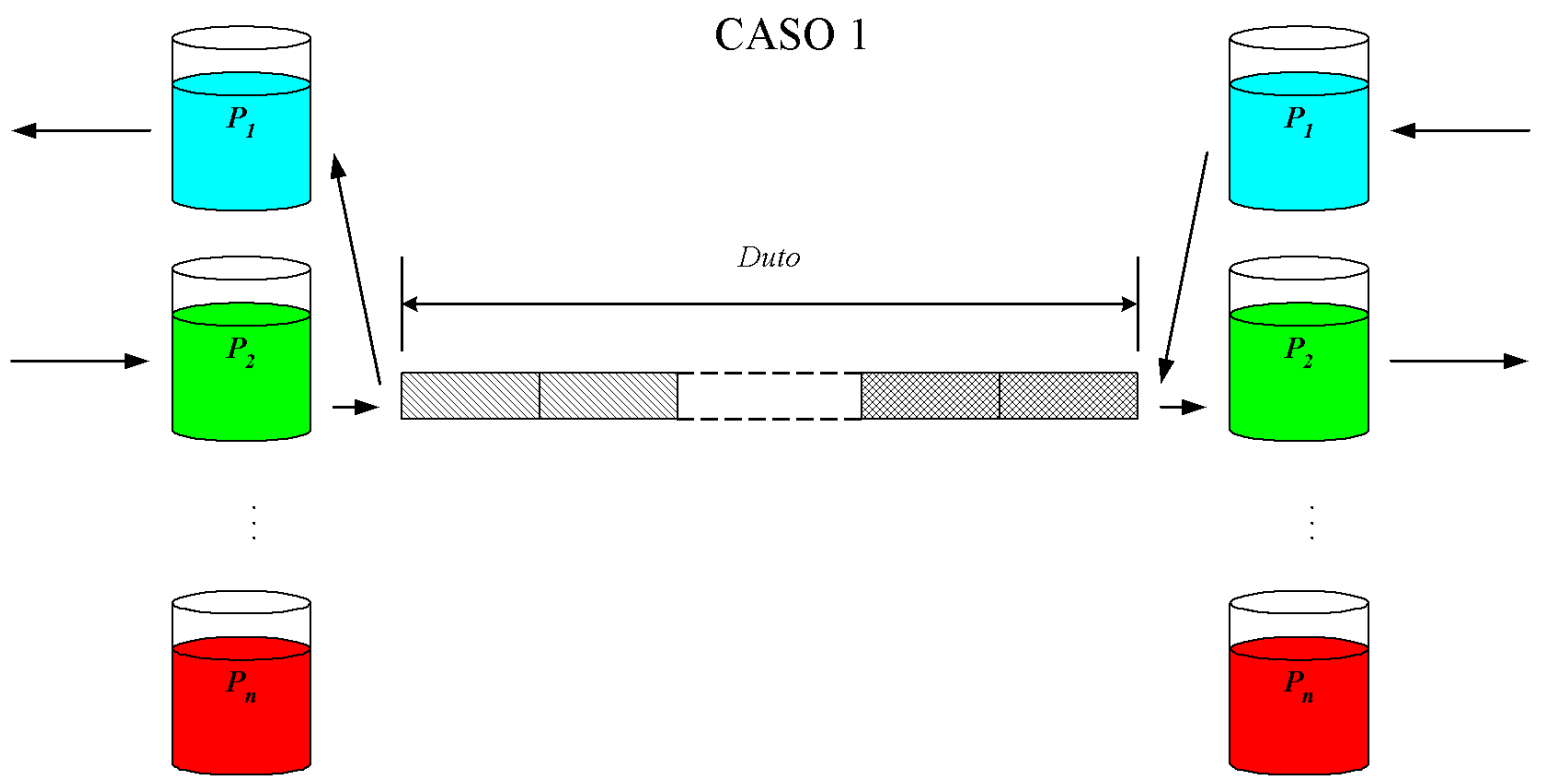

Figura 3.2: Representação do esquema do caso 1 de reversão do duto

Outra situação é: existem a extremidade "A" e a extremidade "B" do duto, e somente há possibilidade de transferir produtos entre as extremidades através do duto, e nas movimentações entre " $A$ " e "B" todas as interfaces geradas são acumuladas em "B", e como não há o consumo em "B" destas interfaces geradas, o volume destas interfaces deve ser movimentado de volta para "A". Por exemplo, a REPLAN envia produtos leves para Barueri através do OPASA; com acúmulo das interfaces geradas (conhecido como resíduo de terminal) das movimentações e pelo espaço limitado para o resíduo em Barueri, há a necessidade de movimentar este resíduo para REPLAN e tratar este resíduo. A figura 3.3 representa a situação descrita acima através de um 
esquema gráfico.

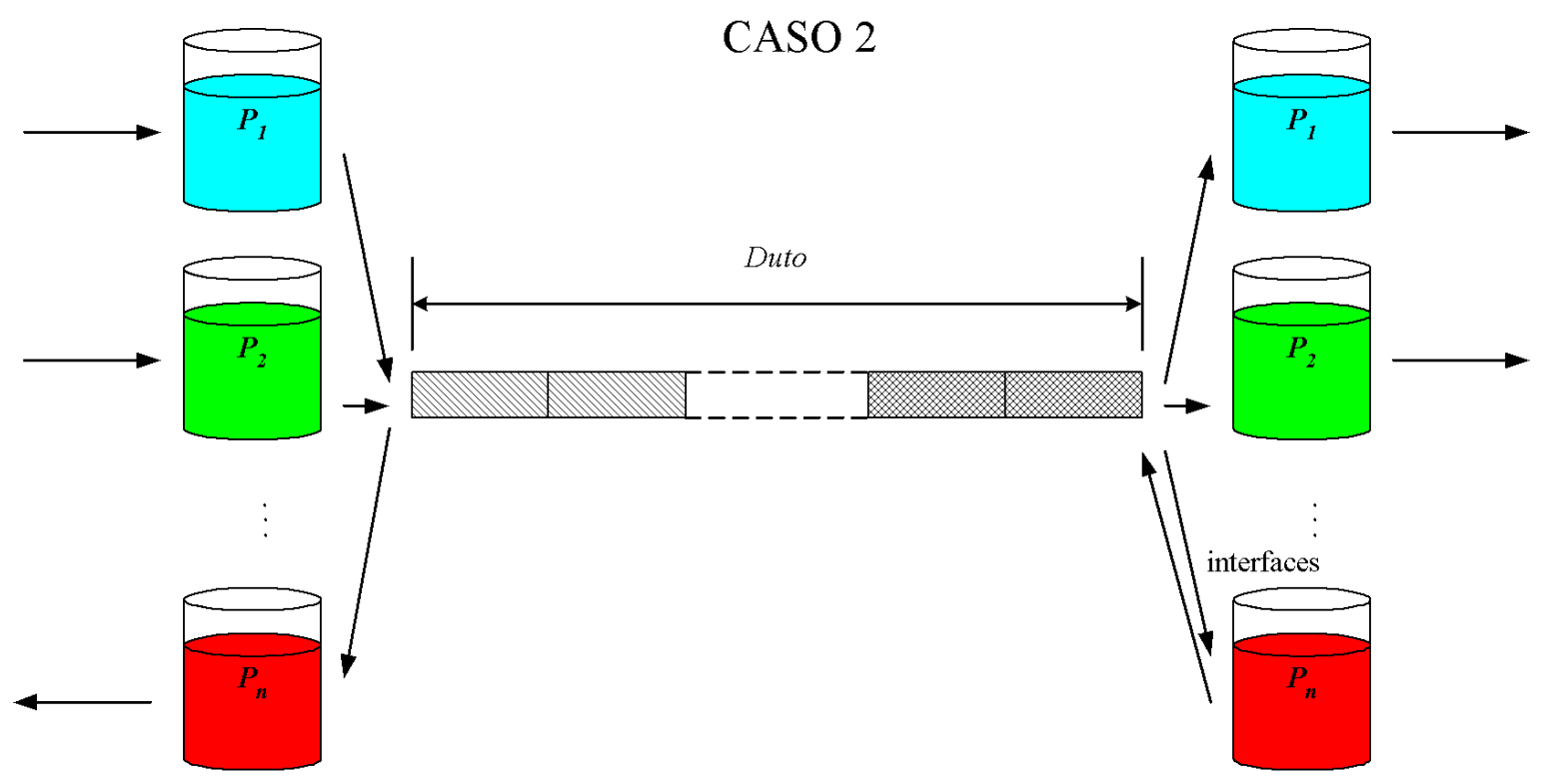

Figura 3.3: Representação do esquema do caso 2 de reversão do duto

Uma outra situação é: existem a extremidade "A" e a extremidade "B" do duto, e somente há possibilidade de transferir produtos entre as extremidades através do duto, e há necessidade de transportar produtos da extremidade "A" para a extremidade "B", e também transportar outros produtos da extremidade "B" para a extremidade "A", e quando o duto ficar parado há necessidade do preenchimento do mesmo ser um produto específico, cuja possibilidade de armazenagem se dá somente em " $A$ " ou há um espaço de armazenamento limitado em "B". Por exemplo, durante a operação da RECAP há a necessidade de carga da unidade de RFCC e produz diluente para óleo combustível; São Caetano recebe carga para o RFCC de outras refinarias e envia diluente de óleo combustível para outras refinarias. Quando há necessidade de parar o duto o produto que deve preenchê-lo é o LCO, que é produzido e consumido na RECAP e São Caetano tem uma pequena capacidade de armazenamento. A figura 3.4 representa a situação descrita acima através de um esquema gráfico. 


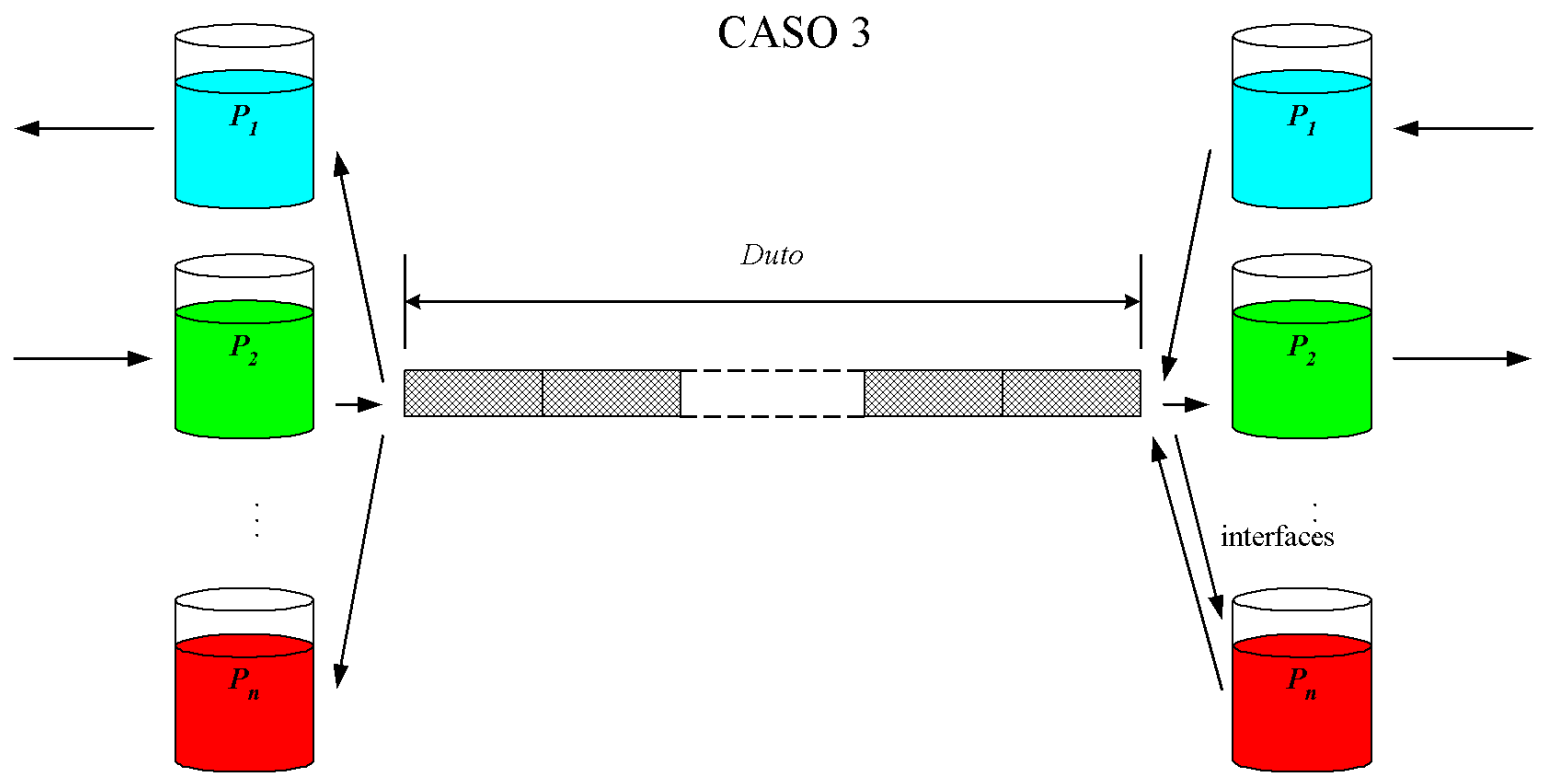

Figura 3.4: Representação do esquema do caso 3 de reversão do duto

Ocorre a reversão de dutos em outras situações; entretanto não serão descritas todas as possibilidades. 


\section{MODELAGEM DO PROBLEMA DE PROGRAMAÇÃO OPERACIONAL DOS DUTOS}

\subsection{INTRODUÇÃO}

Neste capítulo é desenvolvida a modelagem matemática do problema descrito no capítulo anterior. São abordados o desenvolvimento e a estrutura do modelo matemático que representa o problema.

No item 4.2 são apresentadas as considerações gerais do problema. No item 4.3 são apresentadas as hipóteses utilizadas para a formulação. O item 4.4 descreve a estrutura do modelo e está dividido nos seguintes sub-itens: a representação no tempo, as notações para as variáveis e parâmetros, a formulação matemática dos equipamentos e do duto, a formulação das restrições relacionadas ao negócio e a função objetivo.

\subsection{CONSIDERAÇÕES GERAIS}

O modelo apresentado tem como finalidade representar de forma simplificada o comportamento das instalações físicas (equipamentos das refinarias, terminais, estações e dutos), assim como representar os estados destes equipamentos ao longo de um horizonte de tempo.

Os equipamentos representados são os seguintes:

- Duto ou trecho de duto;

- Equipamentos de armazenamento (tanques ${ }^{1}$ ou esferas ${ }^{2}$ );

\footnotetext{
${ }^{1}$ equipamento mais comum que armazena o produto na forma líquida quando a temperatura e pressão são ambientes.

${ }^{2}$ equipamento mais comum que armazena o produto na forma gasosa quando a temperatura e pressão são ambientes.
} 
Os estados dos equipamentos representados ao longo do horizonte de tempo são os seguintes:

- Inventário/estoque do equipamento de armazenamento (produto, quantidade);

- Inventário/estoque do duto ou trecho de duto (produto, quantidade);

- Vazão no equipamento;

- Estado quanto à movimentação (parado ou em operação).

O modelo é baseado naquele desenvolvido por Rejowski Jr (2001). A partir desta formulação são introduzidas variáveis e restrições para representar a possibilidade de inversão do fluxo no duto.

Neste trabalho, o modelo adotado contempla a existência de somente um duto, uma refinaria e um terminal em suas extremidades.

\subsection{HIPÓTESES DO MODELO}

Dentro da formulação do problema, algumas hipóteses são adotadas para o modelamento do problema. Estas hipóteses são as seguintes:

- Condições Iniciais

- A quantidade e a qualidade do inventário/estoque dos equipamentos de armazenamento no instante inicial são conhecidas;

- A quantidade e a qualidade do inventário/estoque dos dutos ou trechos de dutos no instante inicial são conhecidas;

- As movimentações no instante inicial são conhecidas;

- Produção e Demanda

- A produção (quantidades e qualidades) ao longo do horizonte de tempo é conhecida;

- O consumo (quantidades e qualidades) ao longo do horizonte de tempo é conhecido;

- As demandas de envios e recebimentos (quantidades e qualidades) ao longo do horizonte de tempo são conhecidas; 
- Duto

- O deslocamento de produtos ocorre na forma pistonada (plug flow), isto é não há mistura na direção do eixo principal do duto;

- Quando não há movimentação no duto, não há mistura de produto na direção de seu eixo principal;

- Duto é a menor unidade de ligação entre uma refinaria/terminal e refinaria/terminal, ou seja há entrada ou saída de produto somente nas extremidades dos dutos;

- O duto é dividido em volumes iguais denominados lotes;

- Cada lote do duto em cada instante do horizonte de tempo está preenchido com um e somente um produto;

- Escoamento incompressível dos fluidos;

- Em cada intervalo de tempo somente há entrada de produto no duto somente em uma das suas extremidades;

- Em cada intervalo de tempo para cada extremidade do duto há exatamente um único produto se movimentando, quando o duto está operando; $(\mathrm{H} 4.14)$

- A vazão do duto é constante independente do produto bombeado e do produto que está dentro do duto.

- Armazenamento

- O produto a ser armazenado é misturado instantaneamente ao produto anteriormente existente no armazenamento, isto é mistura perfeita;

- O produto que sai dos tanques de armazenamento tem as mesmas características do produto que está anteriormente existente no armazenamento;

- Cada armazenamento (tanque ou esfera) em cada instante do horizonte contém um e somente um produto e também não troca de produto ao longo do horizonte de tempo;

- Para cada produto somente há um armazenamento, isto é os tanques/esferas dos produtos são agregados.

- Gerais

- Todos os produtos estão em fase líquida; 
- Todas as vazões são constantes em cada intervalo de tempo.

\subsection{ESTRUTURA DO MODELO}

O modelo desenvolvido é estruturado de acordo com os seguintes pontos:

- Notações das variáveis e parâmetros;

- Representação do tempo;

- Modelagem que representa o estado dos equipamentos (estoque, vazões, movimentando ou não, etc) ao longo do tempo;

- Modelagem que representa as restrições de capacidade e utilização dos equipamentos ao longo do horizonte de tempo;

- Modelagem que representa as restrições de movimentações ou estados dos equipamentos em razão das restrições de operacionais e de negócio;

- Modelagem da função objetivo do problema.

\subsubsection{NOTAÇÃO}

$\mathrm{Na}$ formulação do problema um item importante é a definição das variáveis do problema. Para organizar a nomenclatura das variáveis contínuas, variáveis binárias, parâmetros contínuos, parâmetros binários e índices, um procedimento de construção do nome foi criado para cada tipo.

Os procedimentos são apresentados a seguir:

- Para índice utilizar somente uma letra minúscula representativa.

- Para as variáveis contínuas utilizar palavras-chaves e alguma informação adicional se houver.

- Para as variáveis binárias utilizar a letra "X", palavras-chaves e alguma informação adicional se houver.

- Para os parâmetros contínuos utilizar a letra "C", palavras-chaves e alguma informação adicional se houver. 
- Para os parâmetros binários utilizar as letras "CX", palavras-chaves e alguma informação adicional se houver.

A tabela 4.1 exemplifica os procedimentos de construção dos nomes.

Tabela 4.1: Procedimento de nomenclatura

\begin{tabular}{|c|c|c|}
\hline Item & Procedimento & Exemplo \\
\hline Índice & letra minúscula & $p \rightarrow$ produto \\
\hline Parâmetro contínuo & $\begin{array}{l}\text { "C" + palavras-chave } \\
+ \text { informação }\end{array}$ & $\begin{array}{l}\text { CVAMIN } \rightarrow \text { Volume mínimo de } \\
\text { armazenamento. }\end{array}$ \\
\hline Parâmetro binário & $\begin{array}{l}\text { "CX" + palavra-chave } \\
+ \text { informação }\end{array}$ & $\begin{array}{l}\text { CXVDI } \rightarrow \text { Indicação do volume } \\
\text { inicial do duto. }\end{array}$ \\
\hline Variável contínua & $\begin{array}{l}\text { palavras-chave + in- } \\
\text { formação }\end{array}$ & $\begin{array}{l}\text { VA } \underline{R} \rightarrow \text { Volume recebido no } \\
\text { armazenamento. }\end{array}$ \\
\hline Variável binária & $\begin{array}{l}\text { "X" + palavra-chave + } \\
\text { informação }\end{array}$ & $\begin{array}{l}X M D \underline{R} \rightarrow \text { Indicação } \\
\text { movimentação de } \\
\underline{\text { duto no sentido }} \text { reverso. }\end{array}$ \\
\hline
\end{tabular}

Os índices são apresentados na tabela 4.2, a qual também apresenta a identificação e a descrição de cada um destes.

Tabela 4.2: Índices utilizados no modelo

\begin{tabular}{|l|l|}
\hline \multicolumn{1}{|c|}{ Índice } & \multicolumn{1}{c|}{ Descrição } \\
\hline$k$ & $\begin{array}{l}\text { Intervalo de tempo. } k=1, \ldots, K, \text { onde } K \text { é o número máximo de intervalos } \\
\text { de tempo. }\end{array}$ \\
\hline$l$ & $\begin{array}{l}\text { Lote de um determinado duto. } l=1, \ldots, L, \text { onde } L \text { é o número máximo de } \\
\text { lotes do duto. }\end{array}$ \\
\hline$p, p^{\prime}$ & Produto. $p=1, \ldots, P$, onde $P$ é o número máximo de produtos. \\
\hline
\end{tabular}


Os parâmetros contínuos são apresentados na tabela 4.3, que também mostra a identificação e a descrição de cada um destes. Os parâmetros binários são apresentados na tabela 4.4, bem como sua identificação e descrição.

Tabela 4.3: Parâmetros contínuos utilizados no modelo

\begin{tabular}{|c|c|}
\hline Parâmetro & Descrição \\
\hline$C V R M I N_{p}$ & Volume mínimo de produto $p$ na refinaria \\
\hline$C V R M A X_{p}$ & Volume máximo de produto $p$ na refinaria \\
\hline$C V R I_{p}$ & Volume inicial de produto $p$ na refinaria \\
\hline$C V R O R_{p, k}$ & $\begin{array}{l}\text { Volume recebido de outros locais na refinaria de produto } p \text { no ins- } \\
\text { tante } k\end{array}$ \\
\hline$C V R O E_{p, k}$ & $\begin{array}{l}\text { Volume enviado para outros locais na refinaria de produto } p \text { no ins- } \\
\text { tante } k\end{array}$ \\
\hline $\operatorname{CVTMIN}_{p}$ & Volume mínimo de produto $p$ no terminal \\
\hline$C V T M A X_{p}$ & Volume máximo de produto $p$ no terminal \\
\hline$C V T I_{p}$ & Volume inicial de produto $p$ no terminal \\
\hline$C_{V T O R}, k$ & $\begin{array}{l}\text { Volume recebido de outros locais (diferente de duto) no terminal de } \\
\text { produto } p \text { no instante } k\end{array}$ \\
\hline$C V T O E_{p, k}$ & $\begin{array}{l}\text { Volume enviado para outros locais (diferente de duto) no terminal de } \\
\text { produto } p \text { no instante } k\end{array}$ \\
\hline$C V D I_{l, p}$ & Volume inicial de produto $p$ no lote $l$ do duto \\
\hline$C V D T$ & Volume total do duto \\
\hline$C V D L$ & Volume de cada lote do duto \\
\hline
\end{tabular}




\begin{tabular}{|l|l|}
\hline Parâmetro & \multicolumn{1}{c|}{ Descrição } \\
\hline$C T$ & Duração do intervalo de tempo \\
\hline$C C E R_{p}$ & Custo de estoque na refinaria do produto $p$ por volume por tempo \\
\hline$C C E T_{p}$ & Custo de estoque no terminal do produto $p$ por volume por tempo \\
\hline$C C E D_{p}$ & Custo de estoque no duto do produto $p$ por volume por tempo \\
\hline$C C B R D_{p}$ & $\begin{array}{l}\text { Custo de bombeamento da refinaria para o duto do produto } p \text { por } \\
\text { volume }\end{array}$ \\
\hline$C C B T D_{p}$ & $\begin{array}{l}\text { Custo de bombeamento do terminal para o duto do produto } p \text { por } \\
\text { volume }\end{array}$ \\
\hline$C C I P_{p, p^{\prime}}$ & Custo da interface entre os produtos $p$ e $p^{\prime}$ \\
\hline
\end{tabular}

Tabela 4.4: Parâmetros binários utilizados no modelo

\begin{tabular}{|l|c|}
\hline Parâmetro & Descrição \\
\hline$C X V D I_{l, p}$ & 1, se o produto $p$ ocupa o lote $l$ do duto no início da operação \\
\hline$C X I P P_{p, p^{\prime}}$ & 1, se a interface entre os produtos $p$ e $p^{\prime}$ é permitida no duto. \\
\hline
\end{tabular}

As variáveis contínuas são apresentadas na tabela 4.5. A mesma tabela mostra a identificação e a descrição de cada uma das variáveis. As variáveis binárias são apresentadas na tabela 4.6, que também mostra a identificação e a descrição de cada uma delas.

Tabela 4.5: Variáveis contínuas utilizadas no modelo

\begin{tabular}{|l|l|}
\hline \multicolumn{1}{|c|}{ Variável } & \multicolumn{1}{c|}{ Descrição } \\
\hline$V R_{p, k}$ & Volume de produto $p$ no instante $k$ na refinaria \\
\hline$V R D R_{p, k}$ & Volume recebido do duto para a refinaria do produto $p$ no instante $k$ \\
\hline$V R D E_{p, k}$ & Volume enviado da refinaria para o duto do produto $p$ no instante $k$ \\
\hline
\end{tabular}




\begin{tabular}{|l|l|}
\hline \multicolumn{1}{|c|}{ Variável } & \multicolumn{1}{c|}{ Descrição } \\
\hline$V T_{p, k}$ & Volume de produto $p$ no instante $k$ no terminal \\
\hline$V T D R_{p, k}$ & Volume recebido do duto para o terminal do produto $p$ no instante $k$ \\
\hline$V T D E_{p, k}$ & Volume enviado do terminal para o duto do produto $p$ no instante $k$ \\
\hline$V D_{l, p, k}$ & Volume ocupado pelo produto $p$, no lote $l$ do duto, no instante $k$ \\
\hline$C U S T O$ & Custo Total \\
\hline
\end{tabular}

Tabela 4.6: Variáveis binárias utilizadas no modelo

\begin{tabular}{|l|l|}
\hline \multicolumn{1}{|c|}{ Variável } & \multicolumn{1}{c|}{ Descrição } \\
\hline$X M D F_{k}$ & $\begin{array}{l}\text { Indica que os produtos dentro do duto estão movimentando na dire- } \\
\text { ção para frente (refinaria } \rightarrow \text { terminal) no instante } k\end{array}$ \\
\hline$X M D R_{k}$ & $\begin{array}{l}\text { Indica que os produtos dentro do duto estão movimentando na dire- } \\
\text { ção reversa (terminal } \rightarrow \text { refinaria) no instante } k\end{array}$ \\
\hline$X M D N_{k}$ & $\begin{array}{l}\text { Indica que os produtos dentro não estão se movimentando no ins- } \\
\text { tante } k \text { dentro do duto }\end{array}$ \\
\hline$X V R D E_{p, k}$ & $\begin{array}{l}\text { Indica que a refinaria está enviando um volume do produto } p \text { para o } \\
\text { duto no instante } k\end{array}$ \\
\hline$X V R D R_{p, k}$ & $\begin{array}{l}\text { Indica que a refinaria está recebendo um volume do produto } p \text { do } \\
\text { duto no instante } k\end{array}$ \\
\hline$X V T D E_{p, k}$ & $\begin{array}{l}\text { Indica que o terminal está enviando um volume do produto } p \text { para o } \\
\text { duto no instante } k\end{array}$ \\
\hline$X V T D R_{p, k}$ & $\begin{array}{l}\text { Indica que o terminal está recebendo um volume do produto } p \text { do } \\
\text { duto no instante } k\end{array}$ \\
\hline Indica que o lote $l$ do duto está ocupado pelo produto $p$ no instante $k$ \\
\hline
\end{tabular}




\begin{tabular}{|c|l|}
\hline Variável & \multicolumn{1}{c|}{ Descrição } \\
\hline$X I P R D_{p, p^{\prime}, k}$ & $\begin{array}{l}\text { Indica que na movimentação refinaria para duto existe uma interface } \\
\text { dos produtos } p \text { e } p^{\prime} \text { no instante } k\end{array}$ \\
\hline$X I P T D_{p, p^{\prime}, k}$ & $\begin{array}{l}\text { Indica que na movimentação terminal para duto existe uma interface } \\
\text { dos produtos } p \text { e } p^{\prime} \text { no instante } k\end{array}$ \\
\hline
\end{tabular}

\subsubsection{REPRESENTAÇÃO DO TEMPO}

Assim como no modelo feito por Rejowski Jr e Pinto (2003), a representação do tempo é de forma discreta. Como explica Smania Filho (2002), esta representação não permite a avaliação de operações com duração inferior ao intervalo de tempo adotado, então o intervalo de tempo a ser adotado a princípio é o intervalo da movimentação (operação) que tem a menor duração de tempo sendo o menor valor do denominador comum entre as operações com o duto e com a tancagem. Considerando isto e as hipóteses H4.15, H4.10 e H4.11, verifica-se que o intervalo de tempo é determinado indiretamente pela razão entre o volume do lote e a vazão do duto. O tempo é representado nesta modelagem com o índice $k$. Na figura 4.1 está ilustrando a relação entre o tempo contínuo (eixo superior) e os valores do índice $k$ (eixo inferior); neste exemplo o intervalo de discretização é de 4 h e $K=16$.

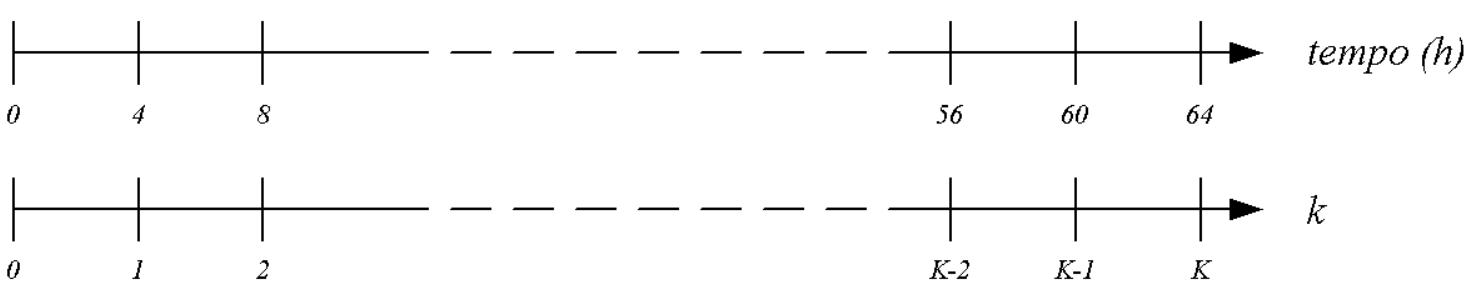

Figura 4.1: Representação da linha do tempo

\subsubsection{MODELOS DOS EQUIPAMENTOS}

Os equipamentos são representados pelas equações de balanço de massa e das limitações físicas tipicamente limitações do tipo de capacidade mínima e máxima. 


\subsubsection{ARMAZENAMENTO}

O equipamento de armazenamento neste problema pode ser tanto um tanque ou uma esfera. É considerado somente um único tipo de equipamento para ambos, denominado de armazenamento.

O balanço de massa para o armazenamento na refinaria é representado por:

$V R_{p, k}=V R_{p, k-1}+\Delta \operatorname{VolRec}_{p, k}-\Delta \operatorname{VolEnv}_{p, k}$ $\forall p, \forall k$

Onde:

- $\Delta \operatorname{VolRec}_{p, k}$ - Volume recebido na refinaria do produto $p$ no instante $k$;

- $\Delta \operatorname{VolEnv}_{p, k}$ - Volume enviado da refinaria do produto $p$ no instante $k$;

O produto $p$ pode ser recebido tanto do duto quanto de demais locais (normalmente da área de refino). Analogamente o envio de produto pode ser feito para o duto ou para outros destinos (por exemplo, mercado consumidores). Assim:

$$
\begin{array}{ll}
V R_{p, k}=V R_{p, k-1}+\left(\Delta \operatorname{VolRD}_{p, k}+\Delta \operatorname{VolRO}_{p, k}\right)-\left(\Delta \operatorname{VolED}_{p, k}+\Delta \operatorname{VolEO}_{p, k}\right) & \forall p, \forall k \\
V R_{p, k}=V R_{p, k-1}+\Delta \operatorname{VolRD}_{p, k}-\Delta \operatorname{VolED}_{p, k}+\Delta \operatorname{VolRO}_{p, k}-\Delta \operatorname{VolEO}_{p, k} & \forall p, \forall k
\end{array}
$$

Onde:

- $\Delta \operatorname{VolRD} D_{p, k}$ - Volume recebido pelo duto na refinaria do produto $p$ no instante $k$;

- $\Delta \operatorname{VolRO}_{p, k}$ - Volume recebido de outros locais (diferente de duto) na refinaria do produto $p$ no instante $k$. Está contido neste volume a produção da refinaria;

- $\Delta$ VolE $D_{p, k}$ - Volume enviado pelo duto na refinaria do produto $p$ no instante $k$;

- $\Delta$ VolE $O_{p, k}$ - Volume enviado para outros (diferente de duto) na refinaria do produto $p$ no instante $k$. Está contido neste volume o consumo interno, vendas locais.

No modelo pode-se escrever as equações de balanço de massa na refinaria no instante inicial (4.1) e nos demais instantes (4.2) da seguinte forma:

$$
\begin{array}{ll}
V R_{p, k}=C V R I_{p}+V R D R_{p, k}-V R D E_{p, k}+V R O R_{p, k}-V R O E_{p, k} & \forall p, k=1 \\
V R_{p, k}=V R_{p, k-1}+V R D R_{p, k}-V R D E_{p, k}+V R O R_{p, k}-V R O E_{p, k} & \forall p, k=2, \ldots, K
\end{array}
$$


Onde:

- $V R D R_{p, k}=\Delta \operatorname{VolRD}_{p, k}$;

- $\operatorname{VROR}_{p, k}=\Delta \operatorname{VolRO}_{p, k}$;

- $V R D E_{p, k}=\Delta \operatorname{VolE} D_{p, k}$;

- $V R O E_{p, k}=\Delta \operatorname{VolE} O_{p, k}$.

Pelas hipóteses H4.4, H4.5 e H4.6, tem-se que $V R O R_{p, k}$ e $V R O E_{p, k}$ são conhecidos ao longo do horizonte de tempo. Então as equações (4.1) e (4.2) são escritas da seguinte forma:

$V R_{p, k}=C V R I_{p}+V R D R_{p, k}-V R D E_{p, k}+C V R O R_{p, k}-C V R O E_{p, k} \quad \forall p, k=1$

$V R_{p, k}=V R_{p, k-1}+V R D R_{p, k}-V R D E_{p, k}+C V R O R_{p, k}-C V R O E_{p, k} \quad \forall p, k=2, \ldots, K$

Outra forma de representar os balanços é combinar as equações (4.3) e (4.4), e rearranjar os termos. Assim tem-se o balanço na forma cumulativa:

$$
\begin{aligned}
& V R_{p, k}=C V R I_{p}+\sum_{k^{\prime} \leq k}\left(V R D R_{p, k^{\prime}}\right)-\sum_{k^{\prime} \leq k}\left(V R D E_{p, k^{\prime}}\right)+\sum_{k^{\prime} \leq k}\left(C V R O R_{p, k^{\prime}}\right)-\sum_{k^{\prime} \leq k}\left(C V R O E_{p, k^{\prime}}\right) \\
& \forall p, \forall k
\end{aligned}
$$

Portanto, as equações (4.3) e (4.4) ou a equação (4.5) representam o volume na refinaria do produto $p$ no instante $k$.

Pelas hipóteses H4.10 e H4.14, os volumes que são trocados entre a refinaria e duto são representados pelas equações (4.6) e (4.7):

$$
\begin{array}{ll}
V R D E_{p, k}=C V D L \cdot X V R D E_{p, k} & \forall p, \forall k \\
V R D R_{p, k}=C V D L \cdot X V R D R_{p, k} & \forall p, \forall k
\end{array}
$$

Para representação dos armazenamentos, além do balanço de massa, as limitações físicas dos armazenamentos são de volume mínimo e volume máximo. As inequações (4.8) e (4.9) representam as limitações de volume.

$$
\begin{array}{ll}
V R_{p, k} \geq C V R M I N_{p} & \forall p, \forall k \\
V R_{p, k} \leq C V R M A X_{p} & \forall p, \forall k
\end{array}
$$


A figura 4.2 ilustra de maneira detalhada as variáveis e parâmetros dos armazenamentos representativos da refinaria.

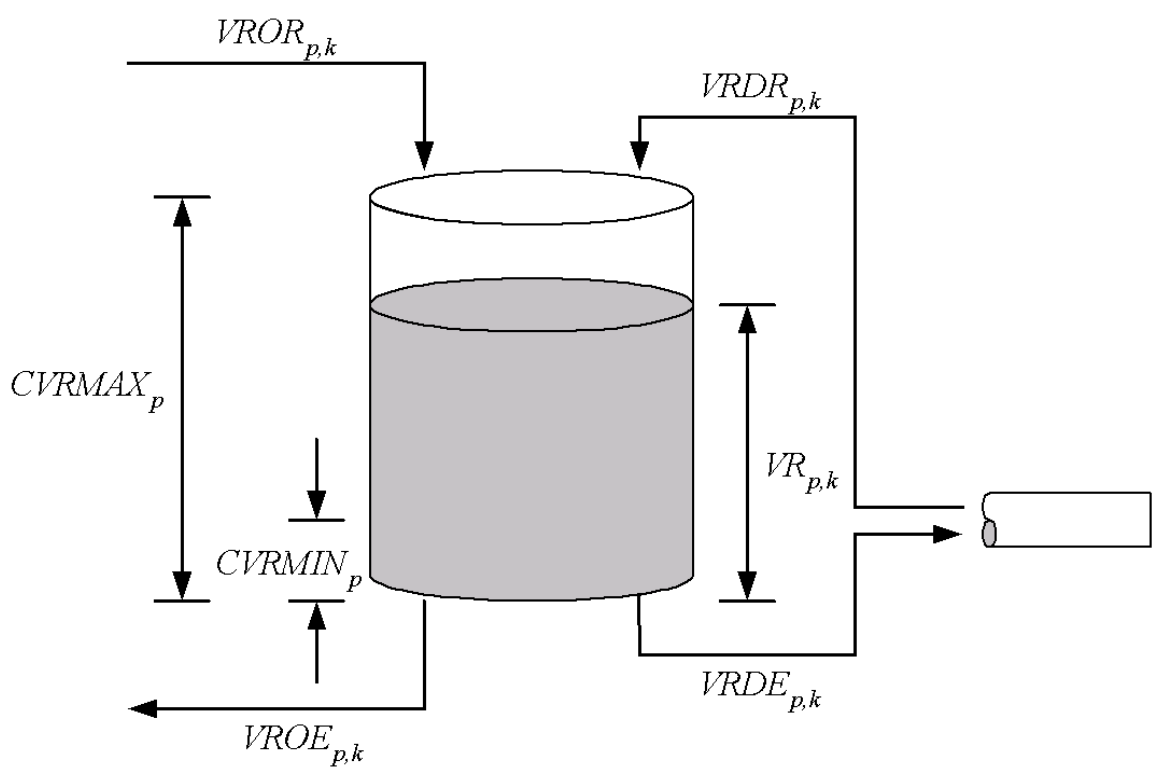

Figura 4.2: Representação do armazenamento na refinaria

De forma análoga pode-se determinar as equações que representam o balanço de massa no terminal e suas restrições. Para o balanço de massa do terminal podem-se utilizar as equações (4.10) e (4.11) ou somente a equação (4.12). As equações (4.13) e (4.14) representam os volumes de troca entre o terminal e o duto. As inequações (4.15) e (4.16) representam os limites de volumes do terminal.

$$
\begin{array}{ll}
V T_{p, k}=C V T I_{p}+V T D R_{p, k}-V T D E_{p, k}+C V T O R_{p, k}-C V T O E_{p, k} & \forall p, k=1 \\
V T_{p, k}=V T_{p, k-1}+V T D R_{p, k}-V T D E_{p, k}+C V T O R_{p, k}-C V T O E_{p, k} & \forall p, k=2, \ldots, K \\
V T_{p, k}=C V T I_{p}+\sum_{k^{\prime} \leq k}\left(V T D R_{p, k^{\prime}}\right)-\sum_{k^{\prime} \leq k}\left(V T D E_{p, k^{\prime}}\right) & \\
\quad+\sum_{k^{\prime} \leq k}\left(C V T O R_{p, k^{\prime}}\right)-\sum_{k^{\prime} \leq k}\left(C V T O E_{p, k^{\prime}}\right) & \forall p, \forall k \\
V T D E_{p, k}=C V D L \cdot X V T D E_{p, k} & \\
V T D R_{p, k}=C V D L \cdot X V T D R_{p, k} & \forall p, \forall k \\
V T_{p, k} \geq C V T M I N_{p} & \forall p, \forall k \\
V T_{p, k} \leq C V T M A X_{p} & \forall p, \forall k
\end{array}
$$

A figura 4.3 ilustra de maneira detalhada as variáveis e parâmetros dos armaze- 
namentos representativos do terminal.

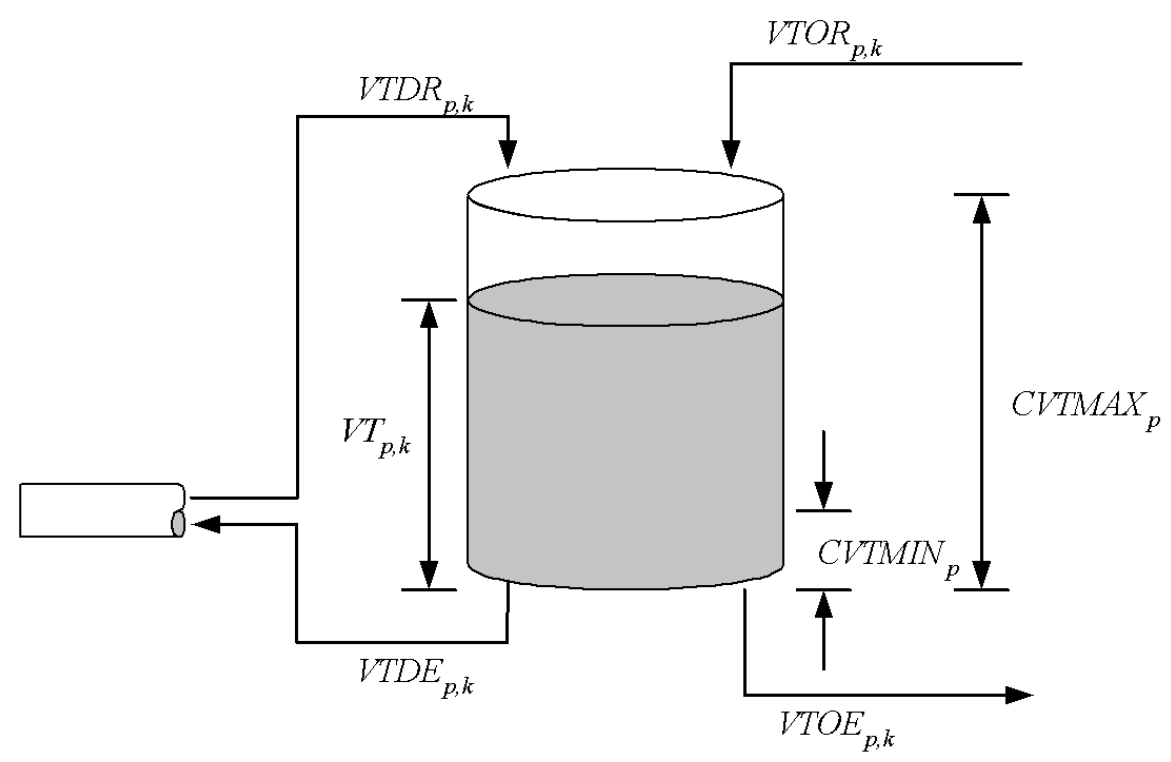

Figura 4.3: Representação do armazenamento no terminal

\subsubsection{DUTOS}

Dutos têm como função básica transferir produtos fluidos entre duas extremidades, que podem ser uma refinaria ou um terminal. O modelo de duto desenvolvido neste problema é similar ao adotado por Rejowski Jr e Pinto (2003). O duto é dividido em lotes de volumes iguais como na hipótese H4.10. As figuras 4.4 e 4.5 ilustram a modelagem do duto quando movimentando algum produto. A figura 4.4 ilustra de maneira detalhada as variáveis e parâmetros do duto quando movimentando da refinaria para o terminal. A figura 4.5 ilustra de maneira detalhada as variáveis e parâmetros do duto quando movimentando do terminal para a refinaria. 

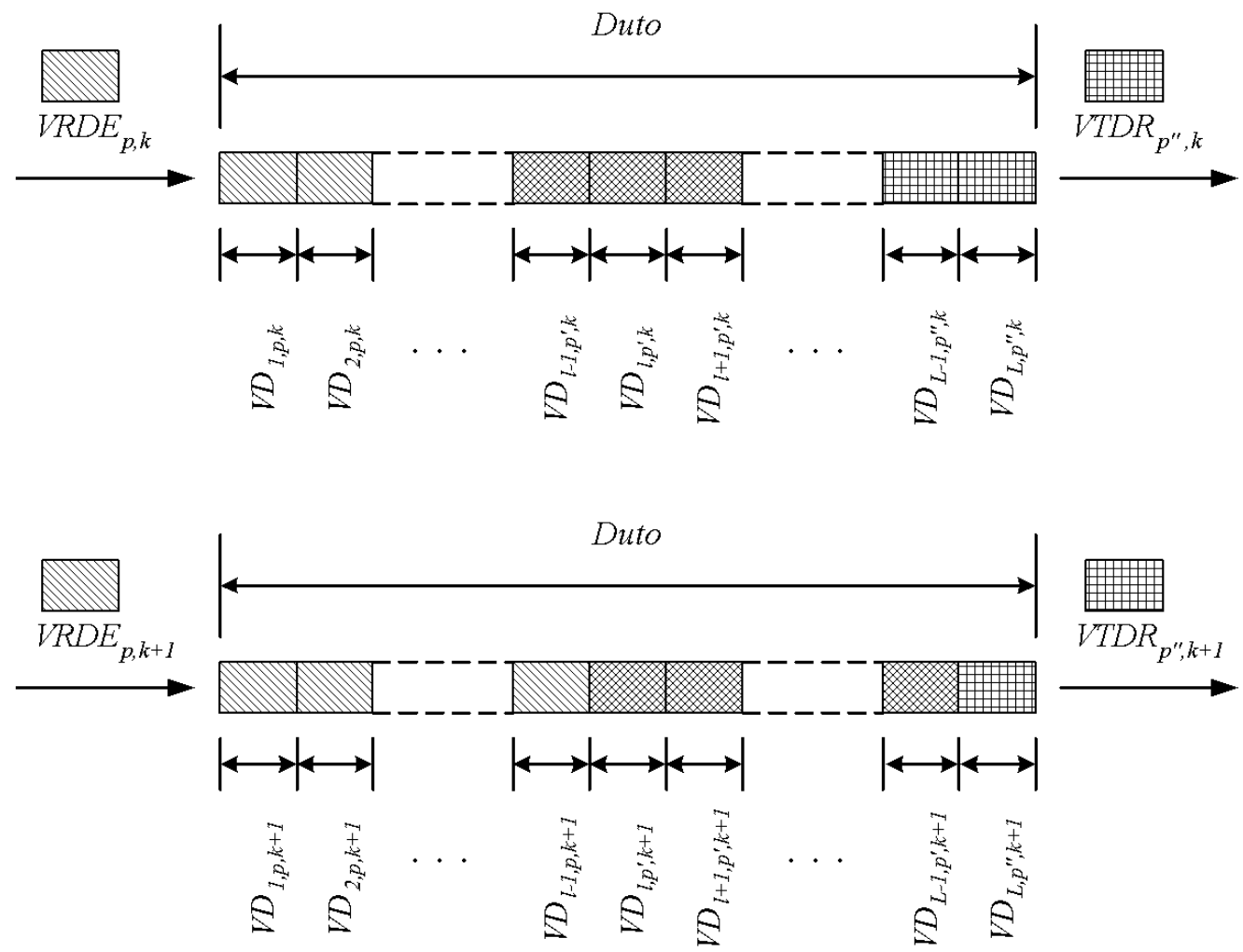

Figura 4.4: Representação do duto movimentando da refinaria para o terminal
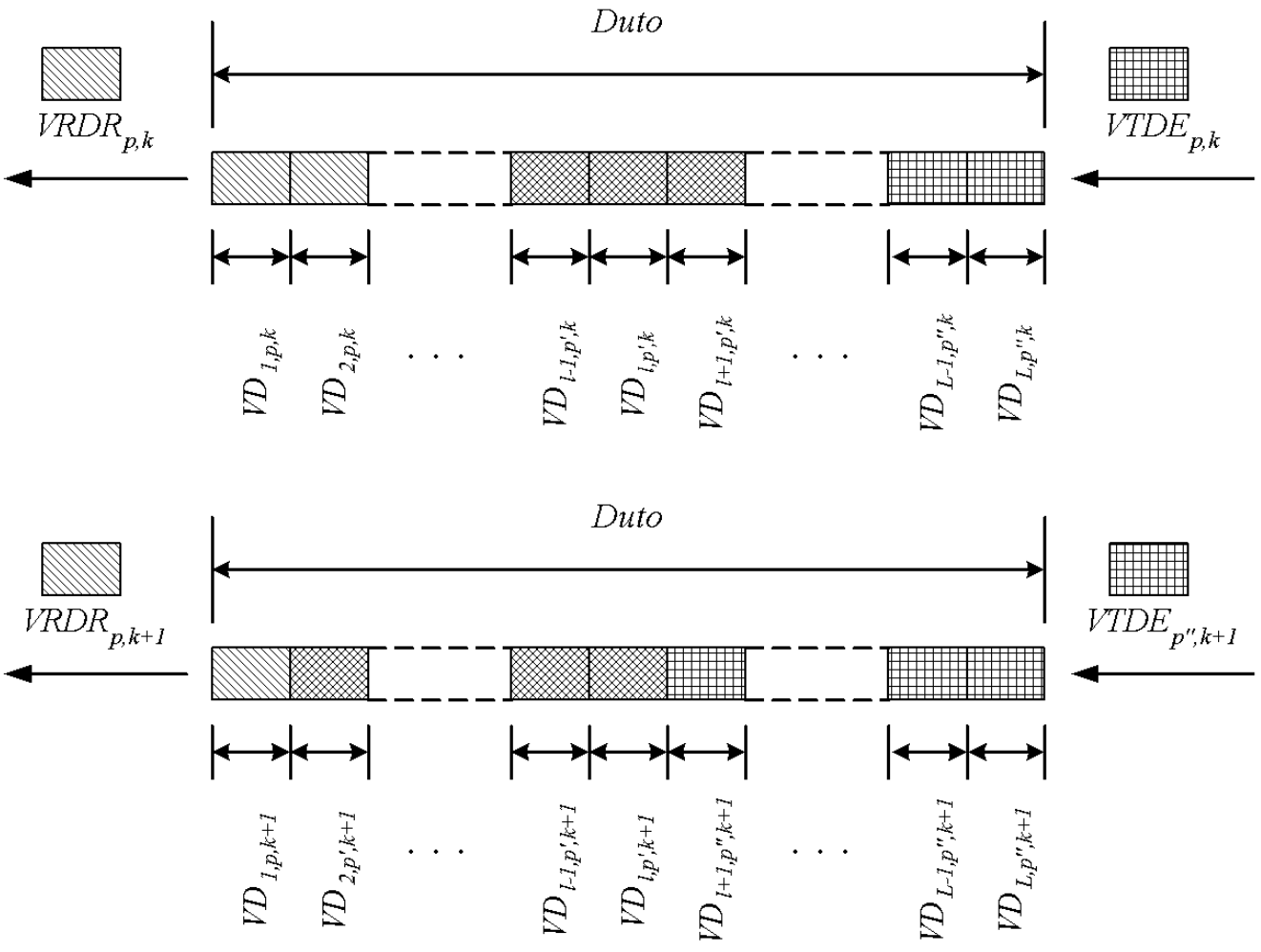

Figura 4.5: Representação do duto movimentando do terminal para a refinaria 
O modelo do duto pode ser representado por três estados (situações) possíveis. primeiro representa a movimentação para frente $\left(X M D F_{k}\right)$, isto é refinaria $\rightarrow$ terminal, o segundo representa a movimentação no sentido reverso $\left(X M D R_{k}\right)$, isto é terminal $\rightarrow$ refinaria e o terceiro denota a não movimentação $\left(X M D N_{k}\right)$. Como expresso na hipótese H4.13, o duto só pode assumir um desses estados. Portanto esta hipótese é representada da seguinte forma:

$X M D F_{k}+X M D R_{k}+X M D N_{k}=1$

$\forall k$

Podemos expressar as demais variáveis e restrições na forma disjuntiva. Nesta forma utilizam-se os operadores ou $(\vee)$, e $(\wedge)$ e negação $(\neg)$.

$$
\left[\begin{array}{c}
X M D F_{k} \\
\vdots
\end{array}\right] \vee\left[\begin{array}{c}
X M D R_{k} \\
\vdots
\end{array}\right] \vee\left[\begin{array}{c}
X M D N_{k} \\
\vdots
\end{array}\right]
$$$$
\forall k
$$

Quando o duto se movimenta para frente $\left(X M D F_{k}=1\right)$ o seguinte conjunto de equações representa o balanço de massa dos lotes do duto é:

$$
X M D F_{k}=1 \Rightarrow V D_{l, p, k}= \begin{cases}C V D I_{l-1, p} & l=2, \ldots, L, \forall p, k=1 \\ V D_{l-1, p, k-1} & l=2, \ldots, L, \forall p, k=2, \ldots, K \\ V R D E_{p, k} & l=1, \forall p, \forall k\end{cases}
$$

Quando o duto se movimenta no sentido reverso $\left(X M D R_{k}=1\right)$ o seguinte conjunto de equações que representa o balanço de massa dos lotes do duto é:

$$
X M D R_{k}=1 \Rightarrow V D_{l, p, k}= \begin{cases}C V D I_{l+1, p} & l=1, \ldots, L-1, \forall p, k=1 \\ V D_{l+1, p, k-1} & l=1, \ldots, L-1, \forall p, k=2, \ldots, K \\ V T D E_{p, k} & l=L, \forall p, \forall k\end{cases}
$$

Quando o duto não se movimenta $\left(X M D N_{k}=1\right)$ o seguinte conjunto de equações que representa o balanço de massa dos lotes do duto é:

$$
X M D N_{k}=1 \Rightarrow V D_{l, p, k}= \begin{cases}C V D I_{l, p} & \forall l, \forall p, k=1 \\ V D_{l, p, k-1} & \forall l, \forall p, k=2, \ldots, K\end{cases}
$$

Pela hipótese H4.10 podem-se representar os volumes dos lotes do duto da se- 
guinte forma:
$V D_{l, p, k}=C V D L \cdot X V D_{l, p, k}$
$\forall l, \forall p, \forall k$
$C V D I_{l, p}=C V D L \cdot C X V D I_{l, p}$
$\forall l, \forall p$

Substituindo as equações (4.6), (4.13), (4.22) e (4.23) nos conjuntos de equações (4.19), (4.20) e (4.21) e eliminando-se $C V D L$, temos:

$X M D F_{k}=1 \Rightarrow X V D_{l, p, k}= \begin{cases}C X V D I_{l-1, p} & l=2, \ldots, L, \forall p, k=1 \\ X V D_{l-1, p, k-1} & l=2, \ldots, L, \forall p, k=2, \ldots, K \\ X V R D E_{p, k} & l=1, \forall p, \forall k\end{cases}$

$X M D R_{k}=1 \Rightarrow X V D_{l, p, k}= \begin{cases}C X V D I_{l+1, p} & l=1, \ldots, L-1, \forall p, k=1 \\ X V D_{l+1, p, k-1} & l=1, \ldots, L-1, \forall p, k=2, \ldots, K \\ X V T D E_{p, k} & l=L, \forall p, \forall k\end{cases}$

$X M D N_{k}=1 \Rightarrow X V D_{l, p, k}= \begin{cases}X C V D I_{l, p} & \forall l, \forall p, k=1 \\ X V D_{l, p, k-1} & \forall l, \forall p, k=2, \ldots, K\end{cases}$

Segundo a hipótese H4.9, são válidos para troca de produtos entre o duto e as extremidades as seguintes equações:

$$
\begin{aligned}
& X M D F_{k}=1 \Rightarrow \begin{cases}X V T D R_{p, k}=C X V D I_{l, p} & l=L, \forall p, k=1 \\
X V T D R_{p, k}=X V D_{l, p, k-1} & l=L, \forall p, k=2, \ldots, K \\
X V R D E_{p, k}=X V D_{l, p, k} & l=1, \forall p, \forall k\end{cases} \\
& X M D R_{k}=1 \Rightarrow \begin{cases}X V R D R_{p, k}=C X V D I_{l, p} & l=1, \forall p, k=1 \\
X V R D R_{p, k}=X V D_{l, p, k-1} & l=1, \forall p, k=2, \ldots, K \\
X V T D E_{p, k}=X V D_{l, p, k} & l=L, \forall p, \forall k\end{cases}
\end{aligned}
$$




$$
X M D N_{k}=1 \Rightarrow \begin{cases}X V R D R_{p, k}=0 & \forall l, \forall p, \forall k \\ X V T D R_{p, k}=0 & \forall l, \forall p, \forall k \\ X V R D E_{p, k}=0 & \forall l, \forall p, \forall k \\ X V T D E_{p, k}=0 & \forall l, \forall p, \forall k\end{cases}
$$

A forma disjuntiva pode ser escrita da seguinte maneira:

$$
\left[\begin{array}{c}
X M D F_{k} \\
\text { eq.(4.24) } \\
\text { eq.(4.27) }
\end{array}\right] \vee\left[\begin{array}{c}
X M D R_{k} \\
\text { eq.(4.25) } \\
\text { eq.(4.28) }
\end{array}\right] \vee\left[\begin{array}{c}
X M D N_{k} \\
\text { eq.(4.26) } \\
\text { eq.(4.29) }
\end{array}\right]
$$

A disjunção em (4.30) deve ser apresentada de uma forma adequada para as técnicas de otimização existentes. A formulação adotada para a transformação da forma disjuntiva para formulação Convex-hull.

O primeiro termo $\left(X M D F_{k}\right)$ da disjunção (4.30), utilizando a técnica de formulação Convex-hull é transformado nas seguintes inequações:

$$
\begin{array}{ll}
X V D_{l, p, k} \geq C X V D I_{l-1, p}+X M D F_{k}-1 & l=2, \ldots, L, \forall p, k=1 \\
X V D_{l, p, k} \geq X V D_{l-1, p, k-1}+X M D F_{k}-1 & l=2, \ldots, L, \forall p, k=2, \ldots, K \\
X V D_{l, p, k} \geq X V R D E_{p, k}+X M D F_{k}-1 & l=1, \forall p, \forall k \\
X V T D R_{p, k} \geq C X V D I_{l, p}+X M D F_{k}-1 & l=L, \forall p, k=1 \\
X V T D R_{p, k} \geq X V D_{l, p, k-1}+X M D F_{k}-1 & l=L, \forall p, k=2, \ldots, K
\end{array}
$$

O segundo termo $\left(X M D R_{k}\right)$ de (4.30), as inequações são:

$$
\begin{array}{ll}
X V D_{l, p, k} \geq C X V D I_{l+1, p}+X M D R_{k}-1 & l=1, \ldots, L-1, \forall p, k=1 \\
X V D_{l, p, k} \geq X V D_{l+1, p, k-1}+X M D R_{k}-1 & l=1, \ldots, L-1, \forall p, k=2, \ldots, K \\
X V D_{l, p, k} \geq X V T D E_{p, k}+X M D R_{k}-1 & l=L, \forall p, \forall k \\
X V R D R_{p, k} \geq C X V D I_{l, p}+X M D R_{k}-1 & l=1, \forall p, k=1 \\
X V R D R_{p, k} \geq X V D_{l, p, k-1}+X M D R_{k}-1 & l=1, \forall p, k=2, \ldots, K
\end{array}
$$


Para o terceiro termo $\left(X M D N_{k}\right)$ de (4.30), as inequações são:

$$
\begin{array}{ll}
X V D_{l, p, k} \geq C X V D I_{l, p}+X M D N_{k}-1 & \forall l, \forall p, k=1 \\
X V D_{l, p, k} \geq X V D_{l, p, k-1}+X M D N_{k}-1 & \forall l, \forall p, k=2, \ldots, K
\end{array}
$$

A partir da hipótese H4.14 que somente pode-se enviar ou receber um único produto ao mesmo tempo, a tem-se a seguinte representação:

$$
\begin{aligned}
& X M D F_{k}=\sum_{p}\left(X V R D E_{p, k}\right) \\
& X M D F_{k}=\sum_{p}\left(X V T D R_{p, k}\right) \\
& X M D R_{k}=\sum_{p}\left(X V T D E_{p, k}\right) \\
& X M D R_{k}=\sum_{p}\left(X V R D R_{p, k}\right)
\end{aligned}
$$

Pela hipótese H4.11 de cada lote ter somente um único produto, tem-se a seguinte representação:

$$
\sum_{p}\left(X V D_{l, p, k}\right)=1 \quad \forall l, \forall k
$$

Portanto para representar o duto o conjunto de equações e inequações são: (4.17), (4.31), (4.32), (4.33), (4.34) e (4.35).

\subsubsection{MODELAGEM DAS RESTRIÇÕES DE NEGÓCIO}

Joly (1999) e Rejowski Jr (2001) propuseram uma formulação para detectar a existência da interface. De forma semelhante é proposta uma formulação para detectar a existência da interface no carregamento em uma das extremidades do duto utilizando os dois primeiros lotes (sentido para frente) ou os dois últimos lotes (sentido reverso). A representação lógica de quando há movimento no duto da refinaria para terminal e existe um produto $p$ no primeiro lote e um produto $p^{\prime}$ no segundo lote, que implica a 
existência de uma interface entre $p$ e $p^{\prime}$, é a seguinte:

$X M D F_{k} \wedge X V D_{1, p, k} \wedge X V D_{2, p^{\prime}, k} \Longrightarrow X I P R D_{p, p^{\prime}, k}$

$\neg\left(X M D F_{k} \wedge X V D_{1, p, k} \wedge X V D_{2, p^{\prime}, k}\right) \vee X I P R D_{p, p^{\prime}, k}$

$\neg X M D F_{k} \vee \neg X V D_{1, p, k} \vee \neg X V D_{2, p^{\prime}, k} \vee X I P R D_{p, p^{\prime}, k}$

Fazendo a transformação da representação lógica para a representação inteira, é a seguinte:

$\left(1-X M D F_{k}\right)+\left(1-X V D_{1, p, k}\right)+\left(1-X V D_{2, p^{\prime}, k}\right)+X I P R D_{p, p^{\prime}, k} \geq 1$

$X I P R D_{p, p^{\prime}, k} \geq X M D F_{k}+X V D_{1, p, k}+X V D_{2, p^{\prime}, k}-2$

Então a formulação que representa a detecção das interfaces quando no sentido para frente (refinaria $\rightarrow$ terminal) é a seguinte:

$$
\begin{array}{ll}
\operatorname{XIPRD}_{p, p^{\prime}, k} \geq X V D_{l, p, k}+X V D_{l+1, p^{\prime}, k}+X M D F_{k}-2 & l=1, \forall p, \forall p^{\prime}, p^{\prime} \neq p, \forall k \\
\operatorname{IIPRD}_{p, p^{\prime}, k} \leq X V D_{l, p, k} & l=1, \forall p, \forall p^{\prime}, p^{\prime} \neq p, \forall k \\
\operatorname{IIPRD}_{p, p^{\prime}, k} \leq X V D_{l+1, p^{\prime}, k} & l=1, \forall p, \forall p^{\prime}, p^{\prime} \neq p, \forall k \\
\operatorname{IIPRD}_{p, p^{\prime}, k} \leq X M D F_{k} & \forall p, \forall p^{\prime}, p^{\prime} \neq p, \forall k
\end{array}
$$

As equações (4.36b), (4.36c) e (4.36d) representam uma formulação mais ajustada para a variável binária $X I P R D_{p, p^{\prime}, k}$.

De forma análoga, a formulação que representa a detecção das interfaces quando no sentido reverso (terminal $\rightarrow$ refinaria) é a seguinte:

$$
\begin{array}{ll}
X I P T D_{p, p^{\prime}, k} \geq X V D_{l, p, k}+X V D_{l-1, p^{\prime}, k}+X M D R_{k}-2 & l=L, \forall p, \forall p^{\prime}, p^{\prime} \neq p, \forall k \\
X I P T D_{p, p^{\prime}, k} \leq X V D_{l, p, k} & l=L, \forall p, \forall p^{\prime}, p^{\prime} \neq p, \forall k \\
X I P T D_{p, p^{\prime}, k} \leq X V D_{l-1, p^{\prime}, k} & l=L, \forall p, \forall p^{\prime}, p^{\prime} \neq p, \forall k \\
X I P T D_{p, p^{\prime}, k} \leq X M D F_{k} & \forall p, \forall p^{\prime}, p^{\prime} \neq p, \forall k
\end{array}
$$

Para evitar algumas seqüências de produtos transportados pelo duto a formulação 
adotada foi a seguinte:

$C X I P P_{p, p^{\prime}} \geq X I P R D_{p, p^{\prime}, k}$

$C X I P P_{p, p^{\prime}} \geq X I P T D_{p, p^{\prime}, k}$

$$
\forall p, p^{\prime} \neq p, \forall k
$$

\subsubsection{FUNÇÃO OBJETIVO}

A função objetivo do problema considera os custos associados ao armazenamento dos produtos na refinaria, no duto e no terminal, os custos associados aos bombeamentos efetuados nas extremidades, e os custos associados as interfaces geradas pelo seqüênciamento de transferência de produtos pelo duto. A formulação da função objetivo é dada por (4.40).

$$
\begin{aligned}
& \text { CUSTO }=\left[\sum_{p=1}^{P} \sum_{k=1}^{K}\left(C C E R_{p} \cdot V R_{p, k}\right)+\sum_{p=1}^{P} \sum_{k=1}^{K}\left(C C E T_{p} \cdot V T_{p, k}\right)\right. \\
& \left.+\sum_{l=1}^{L} \sum_{p=1}^{P} \sum_{k=1}^{K}\left(C C E D_{p} \cdot X V D_{l, p, k} \cdot C V D L\right)\right] \cdot C T \\
& +\left[\sum_{p=1}^{P} \sum_{k=1}^{K}\left(C C B R D_{p} \cdot V R D E_{p, k}\right)+\sum_{p=1}^{P} \sum_{k=1}^{K}\left(C C B T D_{p} \cdot V T D E_{p, k}\right)\right] \\
& +\left[\sum_{p=1}^{P} \sum_{p^{\prime}=1}^{P^{\prime}} \sum_{k=1}^{K}\left(C C I P_{p, p^{\prime}} \cdot X I P R D_{p, p^{\prime}, k}\right)+\sum_{p=1}^{P} \sum_{p^{\prime}=1}^{P^{\prime}} \sum_{k=1}^{K}\left(C C I P_{p, p^{\prime}} \cdot X I P T D_{p, p^{\prime}, k}\right)\right]
\end{aligned}
$$

Esta função linear representa o custo total das operações. Nesta função a primeira parcela representa os custos de estocagem, a segunda parcela representa os custos de bombeamento e a terceira parcela representa os custos das interfaces. 


\subsection{RESUMO DO MODELO}

O modelo proposto (MS) neste estudo é resumido da seguinte forma:

\section{MS:}

Minimizar (4.40)

sujeito a:

(4.3), (4.4), (4.8) e (4.9) restrições de armazenamento da refinaria

(4.6) e (4.7) equações de troca entre refinaria e duto

(4.10), (4.11), (4.15) e (4.16) restrições de armazenamento no terminal

(4.13) e (4.14) equações de troca entre terminal e duto

(4.17), (4.31), (4.32), (4.33), (4.34) e (4.35) restrições que representam os estados do duto

(4.36), (4.37), (4.38) e (4.39) restrições de interface de produtos dentro do duto

$$
\begin{aligned}
& \left(X V R D E_{p, k}, X V T D E_{p, k}\right) \in\{0 ; 1\} \\
& \left(X M D F_{k}, X M D R_{k}, X M D N_{k}\right) \in[0 ; 1] \\
& \left(X V R D R_{p, k}, X V T D R_{p, k}, X V D_{l, p, k}\right) \in[0 ; 1] \\
& \left(X I P R D_{p, p^{\prime}, k}, X I P T D_{p, p^{\prime}, k}\right) \in[0 ; 1] \\
& \left(V R_{p, k}, V R D R_{p, k}, V R D E_{p, k}, V T_{p, k}, V T D R_{p, k}, V T D E_{p, k}, V D_{l, p, k}, C U S T O\right) \geq 0
\end{aligned}
$$

A estrutura resultante é MILP, pois todas as restrições e função objetivo são lineares. As variáveis discretas $X V R D E_{p, k}$ e $X V T D E_{p, k}$ representam as decisões de enviar pelo duto qual produto e quando, e as variáveis contínuas os estados dos equipamentos (volumes e indicações). 


\section{RESULTADOS}

\subsection{MÉTODOS DE SOLUÇÃO}

Para resolução dos problemas MILP, o algoritmo utilizado é o Branch\&Bound $(B \& B)$. O sistema computacional utilizado para resolver o modelo de otimização proposto no capítulo 4 foi o seguinte: GAMS (General Algebraic Modeling System) versão 22.0 (BROOKE et al., 2005), utilizando o solver CPLEX versão 10.0.1, ambos em um microcomputador com 4 processadores Intel Pentium Xeon 4 - $2.4 \mathrm{GHz}, 512 \mathrm{Mb}$ de memória RAM com o sistema operacional Linux.

\subsection{EXEMPLO UTILIZADO}

No item 5.2.1 é descrito o exemplo utilizado. Utiliza-se um protótipo para avaliar o modelo que contém todas as principais características do problema, porém em escala menor. No capítulo 8 é analisado um problema de dimensão real.

\subsubsection{SISTEMA PROTÓTIPO}

Para validar o modelo foi criado um sistema fictício com cenários também fictícios, para validar a modelagem e avaliar o comportamento do modelo sob certas situações. A principal característica deste protótipo é a necessidade de reversão do sentido do duto.

Inicialmente o sistema do protótipo é composto por 3 produtos distintos e a programação se dá ao longo de 16 janelas de tempo que correspondem a 64h de operação:

- tancagem da refinaria. Com 3 tanques, um por produto;

- duto discretizado em 6 lotes de $100 \mathrm{~m}^{3}$ cada;

- tancagem do terminal. Com 3 tanques, um por produto; 
A vazão do duto é de $25 \mathrm{~m}^{3} / \mathrm{h}$.

De acordo com a notação do capítulo 4, os conjuntos que foram utilizados são os seguintes:

Tabela 5.1: Índices do protótipo p/ tancagem da refinaria

\begin{tabular}{|l|l|}
\hline$p$ & $1 \ldots 3 \rightarrow P=3$ \\
\hline$l$ & $1 \ldots 6 \rightarrow L=6$ \\
\hline$k$ & $1 \ldots 16 \rightarrow K=16$ \\
\hline
\end{tabular}

Os parâmetros em relação à tancagem da refinaria que foram utilizados descritos na tabela 5.2 (condição inicial e limites) e 5.3 (recebimentos e envio pré estabelecidos).

Tabela 5.2: Parâmetros do protótipo p/ tancagem da refinaria

\begin{tabular}{|c|c|c|c|}
\hline produto $(p)$ & $C V R I_{p}\left(m^{3}\right)$ & $C V R M I N_{p}\left(m^{3}\right)$ & $C_{V R M A X}\left(m^{3}\right)$ \\
\hline 1 & 1.000 & 500 & 5.000 \\
\hline 2 & 1.280 & 500 & 5.000 \\
\hline 3 & 2.500 & 500 & 5.000 \\
\hline
\end{tabular}

Tabela 5.3: Parâmetros do protótipo p/ tancagem da refinaria ao longo do tempo

\begin{tabular}{|c|c|c|c|}
\hline produto $(p)$ & instante $(k)$ & $C V R O R_{p, k}\left(m^{3}\right)$ & $C V R O E_{p, k}\left(m^{3}\right)$ \\
\hline 1 & $1 \ldots K$ & 60 & 0 \\
\hline 2 & $1 \ldots K$ & 0 & 60 \\
\hline 3 & $1 \ldots K$ & 0 & 0 \\
\hline
\end{tabular}

Os parâmetros em relação à tancagem do terminal que foram utilizados são mostrados nas tabelas 5.4 e 5.5 e definidos de forma análoga àquela das tabelas 5.2 e 5.3:

Tabela 5.4: Parâmetros do protótipo p/ tancagem do terminal

\begin{tabular}{|c|c|c|c|}
\hline produto $(p)$ & $C V T I_{p}\left(m^{3}\right)$ & $\operatorname{CVTMIN}_{p}\left(m^{3}\right)$ & $C V T M A X_{p}\left(m^{3}\right)$ \\
\hline 1 & 1.280 & 500 & 5.000 \\
\hline 2 & 1.000 & 500 & 5.000 \\
\hline 3 & 2.500 & 500 & 5.000 \\
\hline
\end{tabular}


Tabela 5.5: Parâmetros do protótipo p/ tancagem do terminal ao longo do tempo

\begin{tabular}{|c|c|c|c|}
\hline produto $(p)$ & instante $(k)$ & CVTOR $_{p, k}\left(m^{3}\right)$ & CVTOE $_{p, k}\left(m^{3}\right)$ \\
\hline 1 & $1 \ldots K$ & 0 & 60 \\
\hline 2 & $1 \ldots K$ & 60 & 0 \\
\hline 3 & $1 \ldots K$ & 0 & 0 \\
\hline
\end{tabular}

Os parâmetros em relação ao duto que foram utilizados são apresentados nas tabelas 5.6 (volume do duto e volume de cada lote) e 5.7 (preenchimento inicial do duto). Note que inicialmente o duto é preenchido exclusivamente pelo produto 3 .

Tabela 5.6: Parâmetros do protótipo p/ duto

\begin{tabular}{|l|l|}
\hline CVDT & $600 \mathrm{~m}^{3}$ \\
\hline CVDL & $100 \mathrm{~m}^{3}$ \\
\hline
\end{tabular}

Tabela 5.7: Parâmetros do protótipo p/ duto inicial

\begin{tabular}{|c|c|c|}
\hline produto $(p)$ & lote $(l)$ & $C X V D I_{l, p}$ \\
\hline 1 & $l \ldots L$ & 1 \\
\hline 2 & $l \ldots L$ & 0 \\
\hline 3 & $l \ldots L$ & 0 \\
\hline
\end{tabular}

Os parâmetros de custo de estocagem e bombeamento são dados na tabela $5.8 \mathrm{e}$ os custos de interface entre os produtos são dados na tabela 5.9. Note que os custos de estocagem são os mesmos da refinaria, terminal e duto; o mesmo se dá com as custo de interface.

Tabela 5.8: Parâmetros do protótipo p/ custo de estocagem e bombeamento

\begin{tabular}{|c|c|c|c|c|c|}
\hline produto $(p)$ & $\begin{array}{c}C C E R_{p} \\
\left(\mathrm{R} \$ /\left(m^{3} \cdot h\right)\right)\end{array}$ & $\begin{array}{c}C C E T_{p} \\
\left(\mathrm{R} \$ /\left(m^{3} \cdot h\right)\right)\end{array}$ & $\begin{array}{c}C C E D_{p} \\
\left(\mathrm{R} \$ /\left(m^{3} \cdot h\right)\right)\end{array}$ & $\begin{array}{c}C C B R D_{p} \\
\left(\mathrm{R} \$ / m^{3}\right)\end{array}$ & $\begin{array}{c}C C B T D_{p} \\
\left(\mathrm{R} \$ / m^{3}\right)\end{array}$ \\
\hline 1 & 1,0 & 2,0 & 1,5 & 10,0 & 10,0 \\
\hline 2 & 2,0 & 1,0 & 1,5 & 10,0 & 10,0 \\
\hline 3 & 1,5 & 1,5 & 1,5 & 10,0 & 10,0 \\
\hline
\end{tabular}


Tabela 5.9: Parâmetros do protótipo p/ custo da interface

\begin{tabular}{|c|c|c|c|}
\hline \multirow{2}{*}{ CCIP $_{p, p^{\prime}}$} & \multicolumn{3}{|c|}{$(\mathrm{R} \$$ /ocorrência $)$} \\
\cline { 2 - 4 } & 1 & 2 & 3 \\
\hline 1 & 0 & 100 & 100 \\
\hline 2 & 100 & 0 & 100 \\
\hline 3 & 100 & 100 & 0 \\
\hline
\end{tabular}

Este protótipo tem as seguintes características:

- 96 variáveis inteiras $\left(X V R D E_{p, k}\right.$ e $\left.X V T D E_{p, k}\right)$;

- 1.073 variáveis contínuas;

- 1.965 equações e inequações.

\subsubsection{RESULTADOS}

Os resultados otimizados são gerados e comparados com uma solução "manual", conforme apresentado abaixo. A solução "manual" foi obtida utilizando regras heurísticas, onde basicamente se desloca o todo produto 1 que está inicialmente dentro do duto para o terminal pelo bombeando do produto 3 na refinaria, e depois bombeia-se o produto 2 do terminal para a refinaria para não violar o limite de armazenamento do mesmo. O modelo foi resolvido em $21 \mathrm{~h} 51 \mathrm{~min}$ (CPU) de tempo computacional. A tabela 5.10 apresenta a comparação dos valores das funções objetivo manual e otimizada. A tabela 5.11 apresenta a comparação entre os bombeamentos da refinaria para o terminal, e a tabela 5.12 apresenta a comparação entre os bombeamentos do terminal para a refinaria. Note que as tabelas 5.11 e 5.12 indicam os tipos de produto bombeados em cada intervalo de tempo.

Tabela 5.10: Valores da função objetivo

\begin{tabular}{|c|c|}
\hline Caso & Valor Função Objetivo \\
\hline "manual" & 1.212 .100 \\
\hline Modelo & 1.169 .970 \\
\hline
\end{tabular}


Tabela 5.11: Movimentações da refinaria para terminal

\begin{tabular}{|c|c|c|c|c|c|c|c|c|c|c|c|c|c|c|c|c|}
\hline Caso & \multicolumn{11}{|c|}{ tempo $(k)$} \\
& 1 & 2 & 3 & 4 & 5 & 6 & 7 & 8 & 9 & 10 & 11 & 12 & 13 & 14 & 15 & 16 \\
\hline "manual" & 3 & 3 & 3 & 3 & 3 & 3 & & & & & & & & & & \\
\hline Modelo & & & & 2 & 2 & 2 & 3 & & & & & & & & & \\
\hline
\end{tabular}

Tabela 5.12: Movimentações do terminal para refinaria

\begin{tabular}{|c|c|c|c|c|c|c|c|c|c|c|c|c|c|c|c|c|}
\hline \multirow{2}{*}{ Caso } & \multicolumn{10}{|c|}{ tempo $(k)$} \\
& 1 & 2 & 3 & 4 & 5 & 6 & 7 & 8 & 9 & 10 & 11 & 12 & 13 & 14 & 15 & 16 \\
\hline "manual" & & & & & & & 2 & 2 & 2 & 2 & 2 & 2 & 2 & 2 & 3 & 3 \\
\hline Modelo & 1 & 1 & & & & & & 2 & 2 & 3 & 3 & 3 & 3 & 3 & 3 & \\
\hline
\end{tabular}

Os perfis do duto ao longo do horizonte do tempo com o conteúdo do mesmo estão apresentados na tabela 5.13:

Tabela 5.13: Perfis do duto no caso manual e MS

\begin{tabular}{|c|c|c|c|c|c|c|c|c|c|c|c|c|c|c|c|c|}
\hline \multirow{2}{*}{ tempo $(k)$} & \multicolumn{8}{|c|}{ Manual } & \multicolumn{8}{|c|}{ Modelo } \\
\hline & & 1 & 2 & 3 & 4 & 5 & 6 & & & 1 & 2 & 3 & 4 & 5 & 6 & \\
\hline 1 & $\rightarrow$ & 3 & 1 & 1 & 1 & 1 & 1 & & & 1 & 1 & 1 & 1 & 1 & 1 & $\leftarrow$ \\
\hline 2 & $\rightarrow$ & 3 & 3 & 1 & 1 & 1 & 1 & & & 1 & 1 & 1 & 1 & 1 & 1 & $\leftarrow$ \\
\hline 3 & $\rightarrow$ & 3 & 3 & 3 & 1 & 1 & 1 & & & 1 & 1 & 1 & 1 & 1 & 1 & \\
\hline 4 & $\rightarrow$ & 3 & 3 & 3 & 3 & 1 & 1 & & $\rightarrow$ & 2 & 1 & 1 & 1 & 1 & 1 & \\
\hline 5 & $\rightarrow$ & 3 & 3 & 3 & 3 & 3 & 1 & & $\rightarrow$ & 2 & 2 & 1 & 1 & 1 & 1 & \\
\hline 6 & $\rightarrow$ & 3 & 3 & 3 & 3 & 3 & 3 & & $\rightarrow$ & 2 & 2 & 2 & 1 & 1 & 1 & \\
\hline 7 & & 3 & 3 & 3 & 3 & 3 & 2 & $\leftarrow$ & $\rightarrow$ & 3 & 2 & 2 & 2 & 1 & 1 & \\
\hline 8 & & 3 & 3 & 3 & 3 & 2 & 2 & $\leftarrow$ & & 2 & 2 & 2 & 1 & 1 & 2 & $\leftarrow$ \\
\hline 9 & & 3 & 3 & 3 & 2 & 2 & 2 & $\leftarrow$ & & 2 & 2 & 1 & 1 & 2 & 2 & $\leftarrow$ \\
\hline 10 & & 3 & 3 & 2 & 2 & 2 & 2 & $\leftarrow$ & & 2 & 1 & 1 & 2 & 2 & 3 & $\leftarrow$ \\
\hline 11 & & 3 & 2 & 2 & 2 & 2 & 2 & $\leftarrow$ & & 1 & 1 & 2 & 2 & 3 & 3 & $\leftarrow$ \\
\hline 12 & & 2 & 2 & 2 & 2 & 2 & 2 & $\leftarrow$ & & 1 & 2 & 2 & 3 & 3 & 3 & $\leftarrow$ \\
\hline 13 & & 2 & 2 & 2 & 2 & 2 & 2 & $\leftarrow$ & & 2 & 2 & 3 & 3 & 3 & 3 & $\leftarrow$ \\
\hline 14 & & 2 & 2 & 2 & 2 & 2 & 2 & $\leftarrow$ & & 2 & 3 & 3 & 3 & 3 & 3 & $\leftarrow$ \\
\hline 15 & & 2 & 2 & 2 & 2 & 2 & 3 & $\leftarrow$ & & 3 & 3 & 3 & 3 & 3 & 3 & $\leftarrow$ \\
\hline 16 & & 2 & 2 & 2 & 2 & 3 & 3 & & & 3 & 3 & 3 & 3 & 3 & 3 & $\leftarrow$ \\
\hline
\end{tabular}

As figuras referentes aos estoques da refinaria (5.1) e do terminal (5.2) para o caso manual e as figuras referentes aos estoques da refinaria (5.3) e do terminal (5.4) para o caso modelo, são apresentadas abaixo: 
Estoque Refinaria (caso manual)

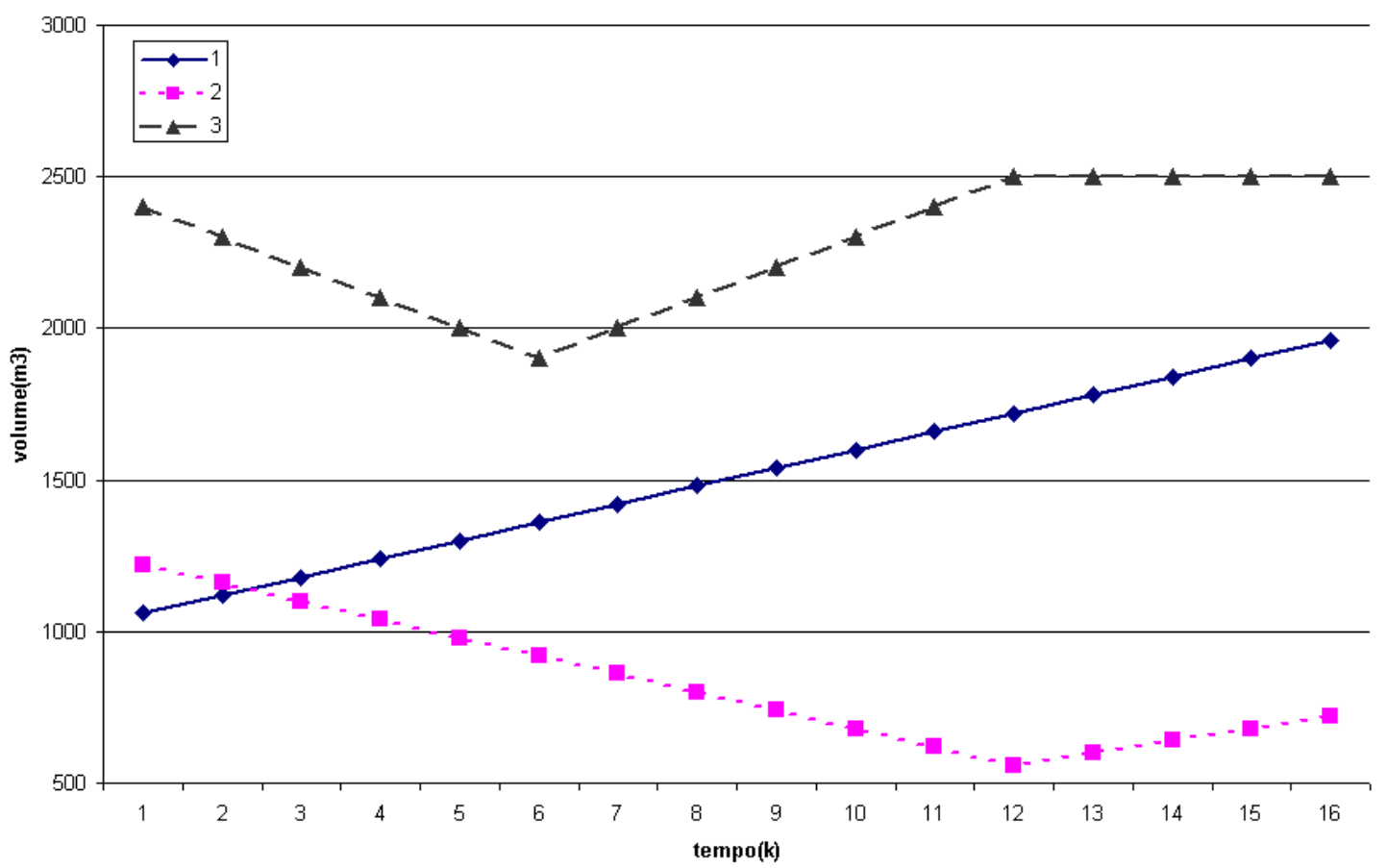

Figura 5.1: Estoque da refinaria para o caso manual

Estoque Terminal (caso manual)

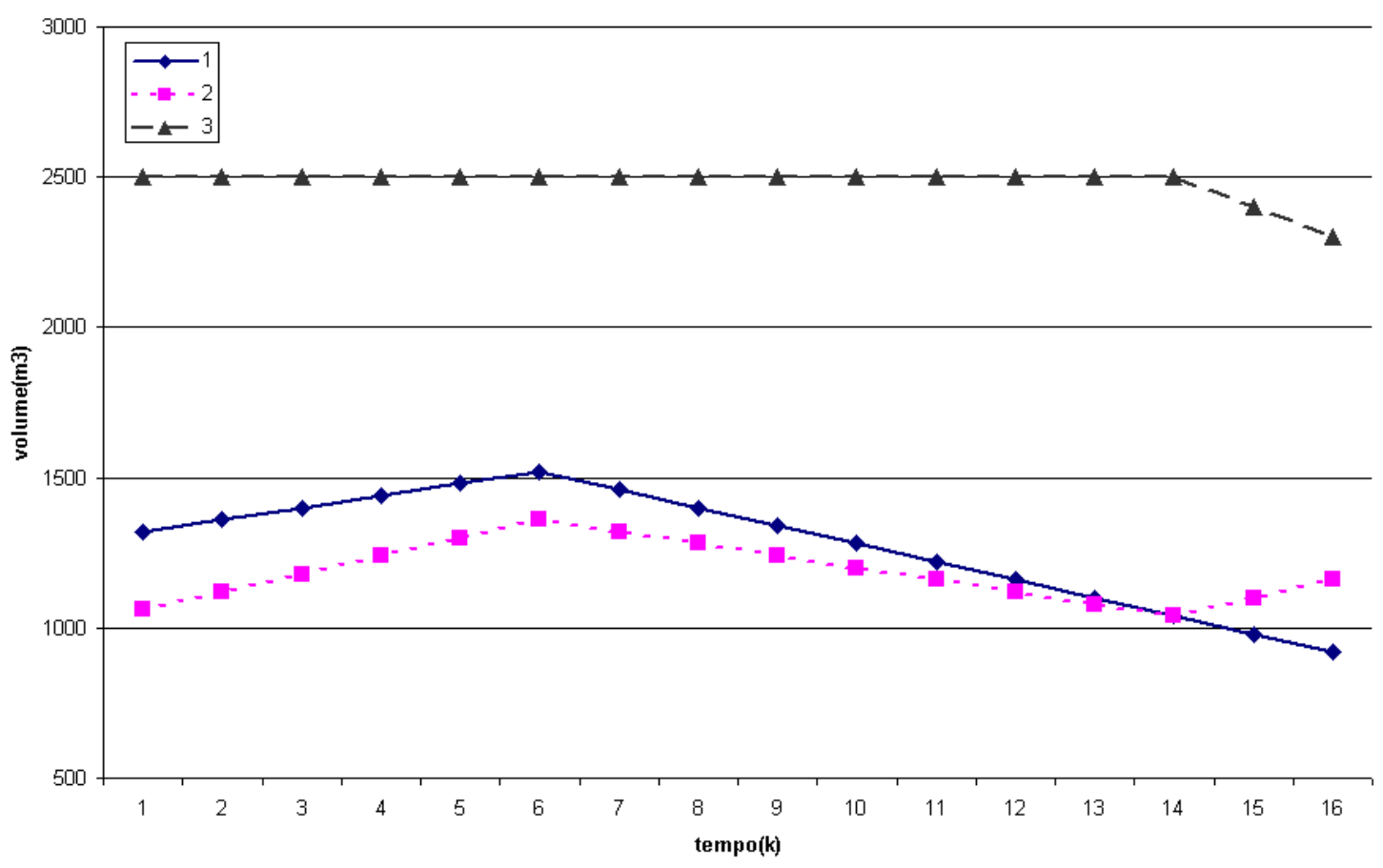

Figura 5.2: Estoque do terminal para o caso manual 
Estoque Refinaria (caso modelo)

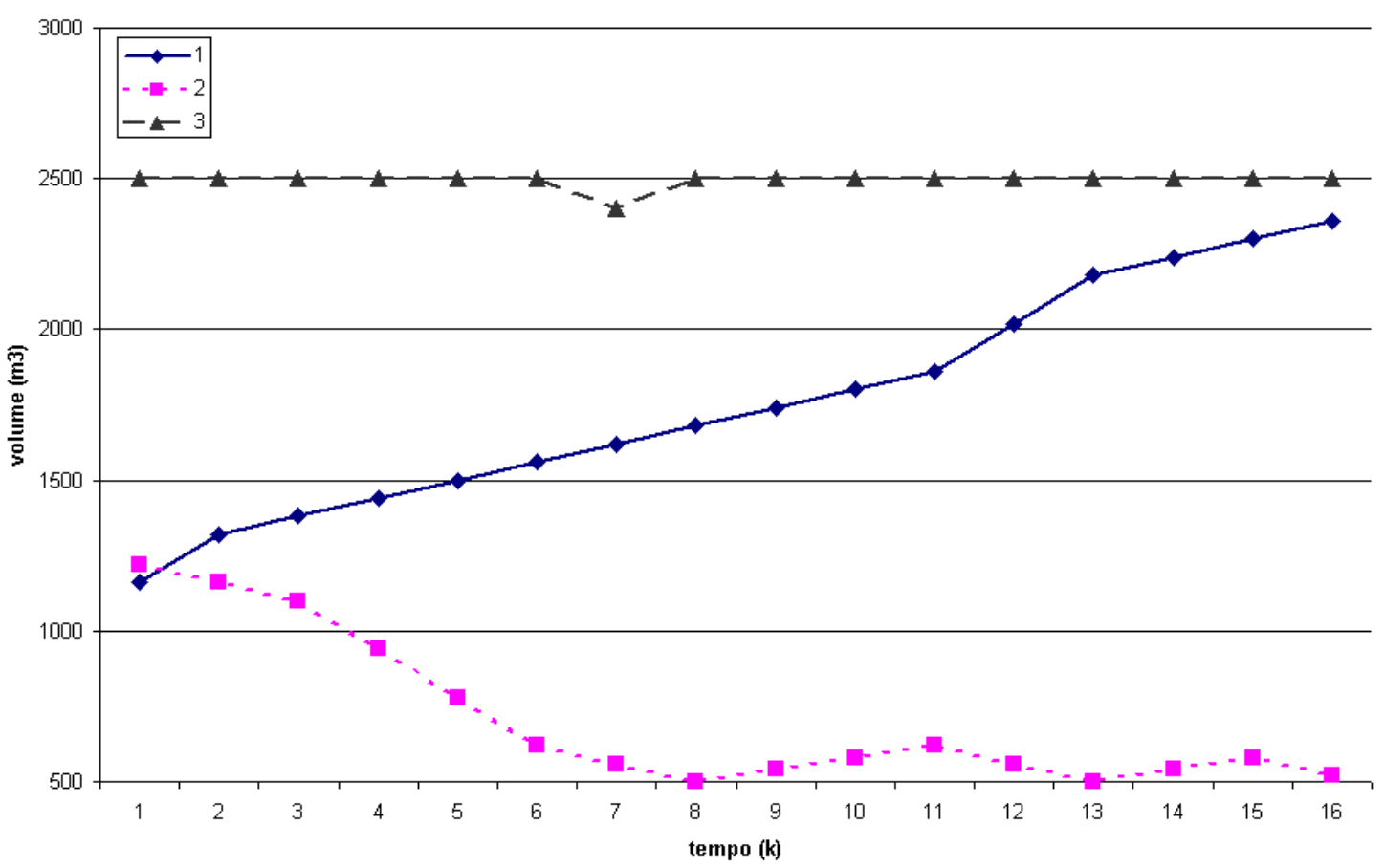

Figura 5.3: Estoque da refinaria para o caso modelo

Estoque Terminal (caso modelo)

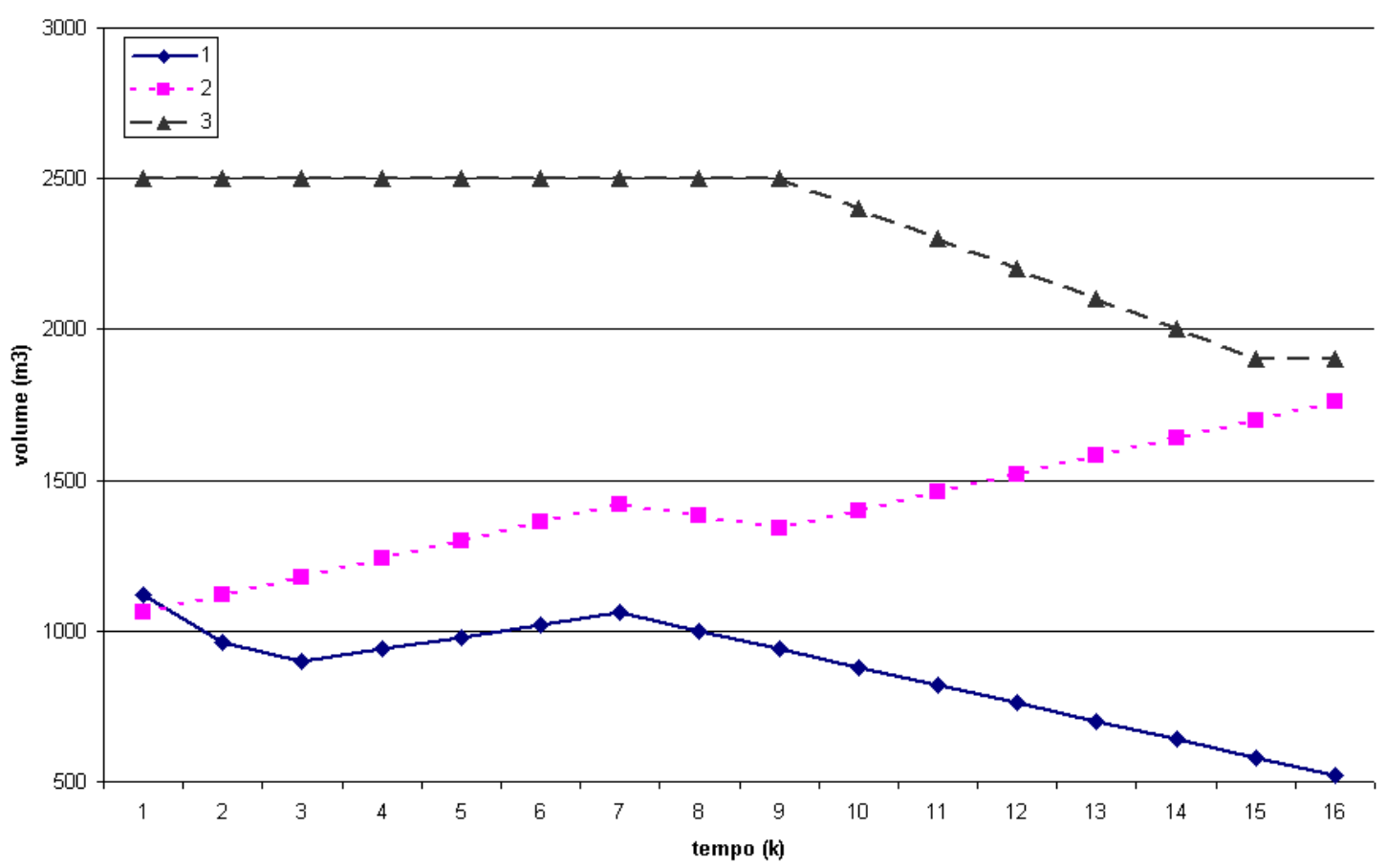

Figura 5.4: Estoque do terminal para o caso modelo 


\subsubsection{DISCUSSÃO DOS RESULTADOS}

Verifica-se que o sistema enviou o produto 1 do terminal para a refinaria nos dois primeiros intervalos, não operando no terceiro intervalo, envia os produtos 2 e 3 da refinaria para o terminal nos intervalos subseqüentes da refinaria para o terminal nos intervalos subseqüentes, dos intervalos 8 a 15 os produtos são enviados do terminal para a refinaria e finalmente não há envio no último intervalo de tempo.

Na solução ótima encontrada, analisando os gráficos de estoque, verifica-se que há uma tendência de minimizar o estoque do produto 2 na refinaria e também minimizar o estoque do produto 1 no terminal, porque o armazenamento destes tem custos maiores do que o estoque do produto 2 no terminal e o estoque do produto 1 na refinaria. Por este motivo nos dois primeiros intervalos o sistema envia o produto 1 do terminal para a refinaria e no final do horizonte o estoque no terminal do produto 1 atinge o mínimo. Pelo mesmo motivo as movimentações do intervalo 4 ao 15 são para minimizar o estoque do produto 2 na refinaria durante um maior número de intervalos possíveis. A opção de bombear o produto 3 nos intervalos 10 a 15 é porque o custo do estoque deste é idêntico em todos os locais. Um ponto interessante é o fato do sistema bombear nos intervalos 4 a 6 o produto 2 , para diminuir o estoque na refinaria e utilizar o duto como "armazenagem" temporária.

O desempenho computacional para solucionar o modelo muito foi elevado para atender às necessidades de um programador. Entretanto, a melhora quanto a função objetivo foi significativa, ao redor de 3,5\%, quando comparado à solução manual.

Uma causa provável para o elevado tempo computacional é a degeneração do modelo, isto é, existência de várias soluções inteiras viáveis mas com o valor próximo da solução ótima. Observando o arquivo de registro do solver (arquivo log), verifica-se que a solução ótima é encontrada no nó 1.905 .204 e com o Gap de 0,53\%, o final da busca ocorre com o nó 3.940.921. Mais da metade da busca pelos nós ocorre somente para provar que a solução ótima. A provável causa é a proximidade dos valores de custo de estocagem do produtos. 


\section{DESENVOLVIMENTO DE RESTRIÇÕES DE CORTE PARA O MODELO MILP}

\subsection{INTRODUÇÃO}

Neste capítulo são desenvolvidas variações na formulação MILP apresentada no capítulo 4. A motivação destas variações são a melhora do tempo computacional para solução do problema.

Cortes válidos são restrições que reduzem a região viável relaxada do modelo sem modificar a região viável inteira do mesmo. Associada à adição de cortes tem-se uma menor enumeração de nós no procedimento Branch\&Bound e a conseqüente redução do esforço computacional.

No item 6.2 são descritos os cortes inteiros válidos baseados naqueles desenvolvidos por Rejowski Jr e Pinto (2003) e no item 6.3 por Rejowski Jr e Pinto (2004), e os resultados associados.

\subsection{CORTE SIMPLES}

\subsubsection{DESCRIÇÃO DE CORTE SIMPLES}

A restrição inteira que corta o espaço de soluções inteiras apresentado por Rejowski Jr e Pinto (2003) se baseia na quantidade mínima necessária de intervalos de tempo que a refinaria deve bombear para o terminal um produto $p$ ao longo do horizonte. Neste modelo consideram-se as mesmas idéias adaptando-se às características do problema. Estas condições dependem do estoque inicial dos produtos na refinaria $\left(C V R I_{p}\right)$ e no terminal $\left(C V T I_{p}\right)$, bem como das quantidades produzidas, das quantidades consumidas e das quantidades transferidas para outros locais de cada produto na refinaria $\left(C V R O E_{p, k}\right.$ e $\left.C V R O R_{p, k}\right)$ e no terminal $\left(C V T O E_{p, k}\right.$ e $\left.C V T O R_{p, k}\right)$. 


\subsubsection{MODELAGEM DE CORTE SIMPLES}

Para formular as restrições de corte simples são inicialmente representados os limites e a formulação.

Os parâmetros são apresentados na tabela 6.1, a qual também apresenta a identificação e a descrição de cada um destes.

Tabela 6.1: Parâmetros associados ao corte simples

\begin{tabular}{|c|l|}
\hline \multicolumn{1}{|c|}{ Parâmetro } & \multicolumn{1}{c|}{ Descrição } \\
\hline CXMDFMIN & $\begin{array}{l}\text { Número mínimo de intervalos de tempo em que o duto está mo- } \\
\text { vimentando produtos da refinaria para o terminal. }\end{array}$ \\
\hline CXMDRMIN & $\begin{array}{l}\text { Número mínimo de intervalos de tempo em que o duto está mo- } \\
\text { vimentando produtos do terminal para a refinaria. }\end{array}$ \\
\hline
\end{tabular}

As formulações para o cálculo do número mínimo de intervalos de tempo são as seguintes:

$$
\begin{aligned}
& \text { CXMDFMIN }=\max \{0, \\
& \max _{p}\left(\left[\frac{\sum_{k=1}^{K}\left(C V T O E_{p, k}-C V T O R_{p, k}\right)-\left(C V T I_{p}-C V T M I N_{p}\right)}{C V D L}\right]\right), \\
& \left.\max _{p}\left(\left\lceil\frac{\sum_{k=1}^{K}\left(C V R O R_{p, k}-C V R O E_{p, k}\right)-\left(C V R M A X_{p}-C V R I_{p}\right)}{C V D L}\right]\right)\right\}
\end{aligned}
$$

$$
\begin{aligned}
& \text { CXMDRMIN }=\max \{0, \\
& \max _{p}\left(\left[\frac{\sum_{k=1}^{K}\left(C V R O E_{p, k}-C V R O R_{p, k}\right)-\left(C V R I_{p}-C V R M I N_{p}\right)}{C V D L}\right]\right), \\
& \left.\max _{p}\left(\left[\frac{\sum_{k=1}^{K}\left(C V T O R_{p, k}-C V T O E_{p, k}\right)-\left(C V T M A X_{p}-C V T I_{p}\right)}{C V D L}\right]\right)\right\}
\end{aligned}
$$


O termo \lceil\rceil nas equações (6.1) e (6.2) representa a função de maior valor inteiro. $\mathrm{Na}$ equação (6.1) o termo $\left(\left[\frac{\sum_{k=1}^{K}\left(C V T O E_{p, k}-C V T O R_{p, k}\right)-\left(C V T I_{p}-C V T M I N_{p}\right)}{C V D L}\right]\right)$ representa a quantidade de intervalos de tempo necessária que o terminal deve receber do produto $p$ ao longo do horizonte de tempo. No numerador, o termo $\sum_{k=1}^{K}\left(C V T O E_{p, k}-\right.$ $\left.C V T O R_{p, k}\right)$ representa a variação de volume do produto $p$ no terminal ao longo do horizonte de operação (quando positiva, significa maior quantidade de produto saindo do que entrando no terminal) e o termo $\left(C V T I_{p}-C V T M I N_{p}\right)$ representa o volume do produto $p$ para atingir o limite mínimo no terminal a partir do instante inicial. A diferença entre estes dois termos, caso o valor seja positiva, representa o volume mínimo necessário do produto $p$ para não violar o volume mínimo no terminal.

Ainda na mesma equação (6.1) o termo $\left(\left[\frac{\sum_{k=1}^{K}\left(C^{2} R O R_{p, k}-C V R O E_{p, k}\right)-\left(C V R M A X_{p}-C V R I_{p}\right)}{C V D L}\right]\right)$ representa a quantidade de intervalos de tempo necessária que a refinaria deve enviar do produto $p$ ao longo do horizonte de tempo. No numerador, o termo $\sum_{k=1}^{K}\left(C V R O R_{p, k}-\right.$ $C V R O E_{p, k}$ ) representa a variação de volume do produto $p$ na refinaria ao longo do horizonte de operação (quando positiva, significa maior quantidade de produto produzido/recebido do que saindo da refinaria) e o termo $\left(C V R M A X_{p}-C V R I_{p}\right)$ representa o espaço disponível do produto $p$ para atingir o volume máximo na refinaria a partir do instante inicial. A diferença entre estes dois termos, caso seja positiva, representa o volume mínimo necessário do produto $p$ para não violar o volume máximo na refinaria.

De modo semelhante pode ser feita a interpretação dos termos da equação (6.2), onde o termo $\left(\left[\frac{\sum_{k=1}^{K}\left(C^{2} R_{0, k}-C V R O R_{p, k}\right)-\left(C V R I_{p}-C V R M I N_{p}\right)}{C V D L}\right]\right)$ representa a quantidade de intervalos de tempo necessária que a refinaria deve receber do produto $p$ ao longo do horizonte de tempo, e o termo $\left(\left[\frac{\sum_{k=1}^{K}\left(\operatorname{CVTOR}_{p, k}-C V T O E_{p, k}\right)-\left(C V T M A X_{p}-C V T I_{p}\right)}{C V D L}\right]\right)$ representa a quantidade de intervalos de tempo necessária que o terminal deve enviar do produto $p$ ao longo do horizonte de tempo, respectivamente.

Então o número mínimo de intervalos de tempo que o duto deve movimentar (em 
ambos os sentidos) produtos são representados da seguinte forma:

$$
\begin{array}{ll}
\sum_{k=1}^{K}\left(X M D F_{k}\right) \geq C X M D F M I N & \text { se CXMDFMIN }>0 \\
\sum_{k=1}^{K}\left(X M D R_{k}\right) \geq C X M D R M I N & \text { se CXMDRMIN }>0
\end{array}
$$

Nota-se que o cálculo do número mínimo de intervalos de tempo é baseado somente em valores conhecidos (equações 6.1 e 6.2), por conseqüência não há necessidade desta formulação fazer parte do modelo. O seu cálculo pode ser previamente executado e somente as equações (6.3) e (6.4) são introduzidas ao modelo.

Portanto o modelo proposto nesta variação (MCS) tem a seguinte forma:

\section{MCS:}

Minimizar (4.40)

sujeito a:

(4.3), (4.4), (4.8) e (4.9) restrições de armazenamento da refinaria (4.6) e (4.7) equações de troca entre refinaria e duto

(4.10), (4.11), (4.15) e (4.16) restrições de armazenamento no terminal (4.13) e (4.14) equações de troca entre terminal e duto

(4.17), (4.31), (4.32), (4.33), (4.34) e (4.35) restrições que representam os estados do duto

(4.36), (4.37), (4.38) e (4.39) restrições de interface de produtos dentro do duto

(6.3) e (6.4) limite da quantidade mínima a ser bombeada

$$
\begin{aligned}
& \left(X V R D E_{p, k}, X V T D E_{p, k}\right) \in\{0 ; 1\} \\
& \left(X M D F_{k}, X M D R_{k}, X M D N_{k}\right) \in[0 ; 1] \\
& \left(X V R D R_{p, k}, X V T D R_{p, k}, X V D_{l, p, k}\right) \in[0 ; 1] \\
& \left(X I P R D_{p, p^{\prime}, k}, X I P T D_{p, p^{\prime}, k}\right) \in[0 ; 1] \\
& \left(V R_{p, k}, V R D R_{p, k}, V R D E_{p, k}, V T_{p, k}, V T D R_{p, k}, V T D E_{p, k}, V D_{l, p, k}, C U S T O\right) \geq 0
\end{aligned}
$$




\subsubsection{RESTRIÇÕES DE CORTE SIMPLES POR INSTANTE DE TEMPO}

Um refinamento possível da modelagem apresentada em (6.1), (6.2), (6.3) e (6.4) é o seguinte: ao invés de gerar um limite da quantidade mínima a ser bombeada para todo o período, definir um limite da quantidade mínima a ser bombeada para cada instante $k$.

Os parâmetros são apresentados na tabela 6.2, a qual também apresenta a identificação e a descrição de cada um destes.

Tabela 6.2: Parâmetros associados ao corte simples por instante de tempo

\begin{tabular}{|c|c|}
\hline Parâmetro & Descrição \\
\hline CXMDFMIN & $\begin{array}{l}\text { Número mínimo de intervalos de tempo em que o duto está movi- } \\
\text { mentando produtos da refinaria para o terminal em cada instante } \\
k \text {. }\end{array}$ \\
\hline$C X M D R M I N_{k}$ & $\begin{array}{l}\text { Número mínimo de intervalos de tempo em que o duto está movi- } \\
\text { mentando produtos do terminal para a refinaria em cada instante } \\
k \text {. }\end{array}$ \\
\hline
\end{tabular}


Os números mínimos de intervalos de tempo são calculados da seguinte forma:

$$
\begin{aligned}
& C X M D F I N_{k}=\max \{0, \\
& \max _{p}\left(\left[\frac{\sum_{k^{\prime}=1}^{k}\left(C V T O E_{p, k^{\prime}}-C V T O R_{p, k^{\prime}}\right)-\left(C V T I_{p}-C V T M I N_{p}\right)}{C V D L}\right]\right), \\
& \left.\max _{p}\left(\left[\frac{\sum_{k^{\prime}=1}^{k}\left(C V R O R_{p, k^{\prime}}-C V R O E_{p, k^{\prime}}\right)-\left(C V R M A X_{p}-C V R I_{p}\right)}{C V D L}\right]\right)\right\} \quad \forall k
\end{aligned}
$$

$$
C X M D R M I N_{k}=\max \{0,
$$

$$
\begin{gathered}
\max _{p}\left(\left[\frac{\sum_{k^{\prime}=1}^{k}\left(C V R O E_{p, k^{\prime}}-C V R O R_{p, k^{\prime}}\right)-\left(C V R I_{p}-C V R M I N_{p}\right)}{C V D L}\right]\right), \\
\left.\max _{p}\left(\left[\frac{\sum_{k^{\prime}=1}^{k}\left(C V T O R_{p, k^{\prime}}-C V T O E_{p, k^{\prime}}\right)-\left(C V T M A X_{p}-C V T I_{p}\right)}{C V D L}\right]\right)\right\} \quad \forall k
\end{gathered}
$$

Então os intervalos de tempo em que os produtos devem ser bombeados são representados da seguinte forma:

$$
\begin{aligned}
& \sum_{k^{\prime}=1}^{k}\left(X M D F_{k^{\prime}}\right) \geq C X M D F M I N_{k} \quad \forall k \in\left(\operatorname{CXMDFMIN}_{k}>0\right) \\
& \sum_{k^{\prime}=1}^{k}\left(X M D R_{k^{\prime}}\right) \geq C X M D R M I N_{k} \quad \forall k \in\left(\operatorname{CXMDRMIN}_{k}>0\right)
\end{aligned}
$$


Portanto o modelo proposto nesta variação (MCST) tem a seguinte estrutura:

\section{MCST:}

Minimizar (4.40)

sujeito a:

(4.3), (4.4), (4.8) e (4.9) restrições de armazenamento da refinaria

(4.6) e (4.7) equações de troca entre refinaria e duto

(4.10), (4.11), (4.15) e (4.16) restrições de armazenamento no terminal

(4.13) e (4.14) equações de troca entre terminal e duto

(4.17), (4.31), (4.32), (4.33), (4.34) e (4.35) restrições que representam os estados do duto

(4.36), (4.37), (4.38) e (4.39) restrições de interface de produtos dentro do duto

(6.7) e (6.8) limite da quantidade mínima a ser bombeada

$$
\begin{aligned}
& \left(X V R D E_{p, k}, X V T D E_{p, k}\right) \in\{0 ; 1\} \\
& \left(X M D F_{k}, X M D R_{k}, X M D N_{k}\right) \in[0 ; 1] \\
& \left(X V R D R_{p, k}, X V T D R_{p, k}, X V D_{l, p, k}\right) \in[0 ; 1] \\
& \left(X I P R D_{p, p^{\prime}, k}, X I P T D_{p, p^{\prime}, k}\right) \in[0 ; 1] \\
& \left(V R_{p, k}, V R D R_{p, k}, V R D E_{p, k}, V T_{p, k}, V T D R_{p, k}, V T D E_{p, k}, V D_{l, p, k}, C U S T O\right) \geq 0
\end{aligned}
$$

\subsection{CORTE COMPLETO}

\subsubsection{DESCRIÇÃO DE CORTE COMPLETO}

O corte inteiro apresentado por Rejowski Jr e Pinto (2004) é baseado na mesma idéia descrita no item 6.2.1, no entanto é aprimorado porque contabiliza no cálculo da quantidade mínima de movimentações o volume do duto e os produtos que estão inicialmente dentro do duto para diversas situações da operação. 


\subsubsection{MODELAGEM DE CORTE COMPLETO}

A formulação dos cortes, para cada sentido do duto (frente ou reverso), é separada em seis partes :

- A quantidade mínima do produto $p$ a ser enviada da refinaria (ou do terminal);

- A quantidade mínima do produto $p$ a ser recebida no terminal (ou na refinaria);

- A quantidade de lotes para deslocar o produto $p$ a ser recebida no terminal (ou na refinaria) originada do duto;

- A quantidade de produto $p$ a ser recebida no terminal (ou na refinaria) originada do duto;

- A quantidade de lotes para deslocar o produto $p$ a ser recebida no terminal (ou na refinaria) originada na refinaria (ou do terminal);

- A quantidade de produto $p$ a ser recebida no terminal (ou na refinaria) originada da refinaria (ou do terminal).

Para descrever a formulação, os parâmetros da tabela 6.3 são utilizados.

Tabela 6.3: Parâmetros associados ao corte completo

\begin{tabular}{|l|l|}
\hline \multicolumn{1}{|c|}{ Parâmetro } & \multicolumn{1}{c|}{ Descrição } \\
\hline$C X V R D E M I N_{p}$ & $\begin{array}{l}\text { Quantidade mínima de lotes do produto } p \text { que a refinaria deve } \\
\text { enviar para o duto. }\end{array}$ \\
\hline$C X V T D R M I N_{p}$ & $\begin{array}{l}\text { Quantidade mínima de lotes do produto } p \text { que o terminal deve } \\
\text { receber do duto. }\end{array}$ \\
\hline$C X V D T D_{p}$ & $\begin{array}{l}\text { Quantidade de lotes para iniciar o recebimento do produto } p \text { no } \\
\text { terminal que tem como origem o duto. }\end{array}$ \\
\hline$C X V D T R_{p}$ & $\begin{array}{l}\text { Quantidade de lotes do produto } p \text { a ser recebida no terminal ori- } \\
\text { ginada do duto. }\end{array}$ \\
\hline
\end{tabular}




\begin{tabular}{|c|c|}
\hline Parâmetro & Descrição \\
\hline$C X V D T T D_{p}$ & $\begin{array}{l}\text { Quantidade total de lotes para movimentar o produto } p \text { a ser re- } \\
\text { cebida no terminal originada do duto. }\end{array}$ \\
\hline$C X V R T D_{p}$ & $\begin{array}{l}\text { Quantidade de lotes para iniciar o recebimento do produto } p \text { no } \\
\text { terminal que tem como origem a refinaria. }\end{array}$ \\
\hline$C X V R T R_{p}$ & $\begin{array}{l}\text { Quantidade de lotes do produto } p \text { a ser recebida no terminal ori- } \\
\text { ginada na refinaria. }\end{array}$ \\
\hline$C X V T D E M I N_{p}$ & $\begin{array}{l}\text { Quantidade mínima de lotes do produto } p \text { que o terminal deve } \\
\text { enviar para o duto. }\end{array}$ \\
\hline$C X V R D R M I N_{p}$ & $\begin{array}{l}\text { Quantidade mínima de lotes do produto } p \text { que a refinaria deve } \\
\text { receber do duto. }\end{array}$ \\
\hline$C X V D R D_{p}$ & $\begin{array}{l}\text { Quantidade de lotes para iniciar o recebimento do produto } p \text { na } \\
\text { refinaria que tem como origem o duto. }\end{array}$ \\
\hline$C X V D R R_{p}$ & $\begin{array}{l}\text { Quantidade de lotes do produto } p \text { a ser recebida na refinaria ori- } \\
\text { ginada do duto. }\end{array}$ \\
\hline$C X V D R T D_{p}$ & $\begin{array}{l}\text { Quantidade total de lotes para movimentar o produto } p \text { a ser re- } \\
\text { cebida na refinaria originada do duto. }\end{array}$ \\
\hline$C X V T R D_{p}$ & $\begin{array}{l}\text { Quantidade de lotes para iniciar o recebimento do produto } p \text { na } \\
\text { refinaria que tem como origem o terminal. }\end{array}$ \\
\hline$C X V T R R_{p}$ & $\begin{array}{l}\text { Quantidade de lotes do produto } p \text { a ser recebida na refinaria ori- } \\
\text { ginada no terminal. }\end{array}$ \\
\hline$C X V M R D$ & $\begin{array}{l}\text { Quantidade de lotes para movimentar os produtos da refinaria } \\
\text { para dentro do duto. }\end{array}$ \\
\hline$C X V M D T$ & $\begin{array}{l}\text { Quantidade de lotes para movimentar os produtos contidos no } \\
\text { duto para o terminal. }\end{array}$ \\
\hline$C X V M R T$ & $\begin{array}{l}\text { Quantidade de lotes para movimentar os produtos da refinaria } \\
\text { para o terminal. }\end{array}$ \\
\hline
\end{tabular}




\begin{tabular}{|l|l|}
\hline \multicolumn{1}{|c|}{ Parâmetro } & \multicolumn{1}{c|}{ Descrição } \\
\hline$C X V M T D$ & $\begin{array}{l}\text { Quantidade de lotes para movimentar os produtos do terminal } \\
\text { para dentro do duto. }\end{array}$ \\
\hline$C X V M D R$ & $\begin{array}{l}\text { Quantidade de lotes para movimentar os produtos contidos no } \\
\text { duto para a refinaria. }\end{array}$ \\
\hline$C X V M T R$ & $\begin{array}{l}\text { Quantidade de lotes para movimentar os produtos do terminal } \\
\text { para a refinaria. }\end{array}$ \\
\hline$C S I T R T_{p}$ & $\begin{array}{l}\text { Identificação de uma situação para o produto } p \text { na movimentação } \\
\text { entre a refinaria e o terminal. }\end{array}$ \\
\hline$C S I T T R_{p}$ & $\begin{array}{l}\text { Identificação de uma situação para o produto } p \text { na movimentação } \\
\text { entre o terminal e a refinaria. }\end{array}$ \\
\hline
\end{tabular}

Existem 4 situações possíveis para as movimentações da refinaria para o terminal para o produto $p$. Conforme figuras 6.1 a 6.4 .

Situação 1 - Nesta situação a refinaria tem uma quantidade excedente do produto p. A figura 6.1 ilustra esta situação.

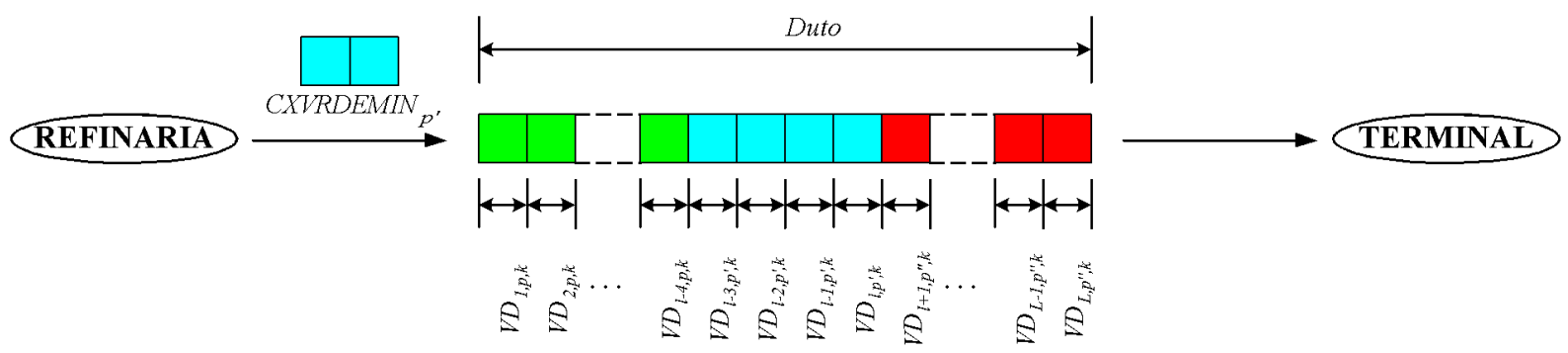

Figura 6.1: Ilustração da situação 1 para corte completo

Situação 2 - Nesta situação o terminal necessita de uma quantidade do produto $p$, e no instante inicial esta quantidade é menor que a quantidade que está presente dentro do duto. A figura 6.2 ilustra esta situação. 


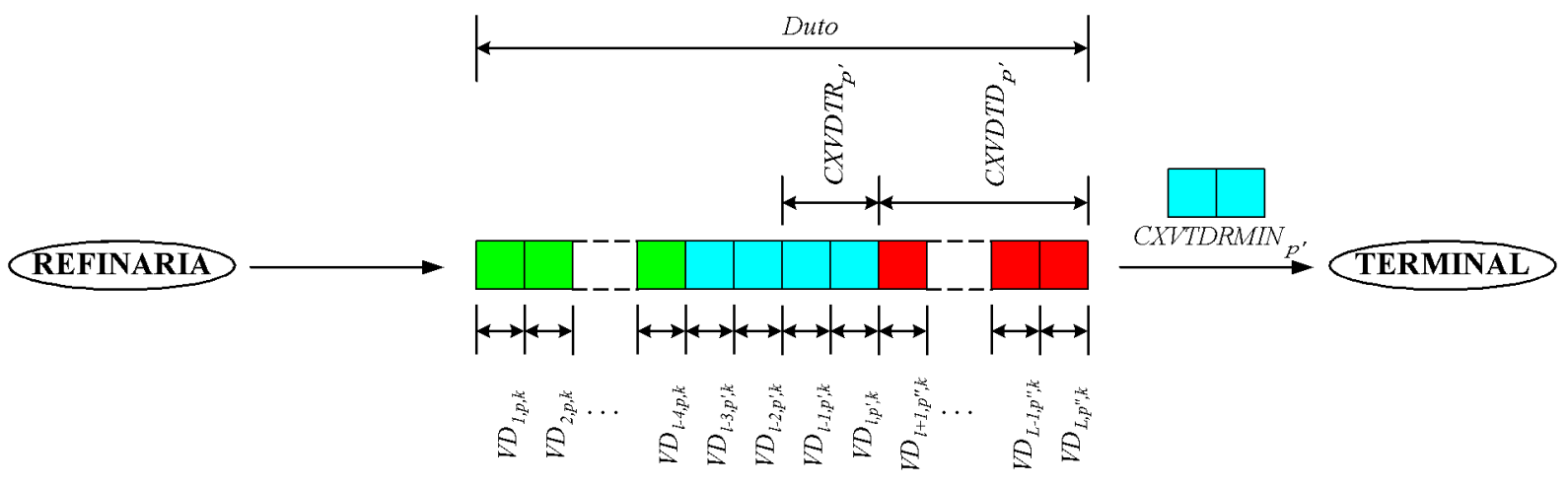

Figura 6.2: Ilustração da situação 2 para corte completo

Situação 3 - Nesta situação o terminal necessita de uma quantidade do produto $p$, e no instante inicial esta quantidade é exatamente igual à quantidade que está presente dentro do duto. A figura 6.3 ilustra esta situação.

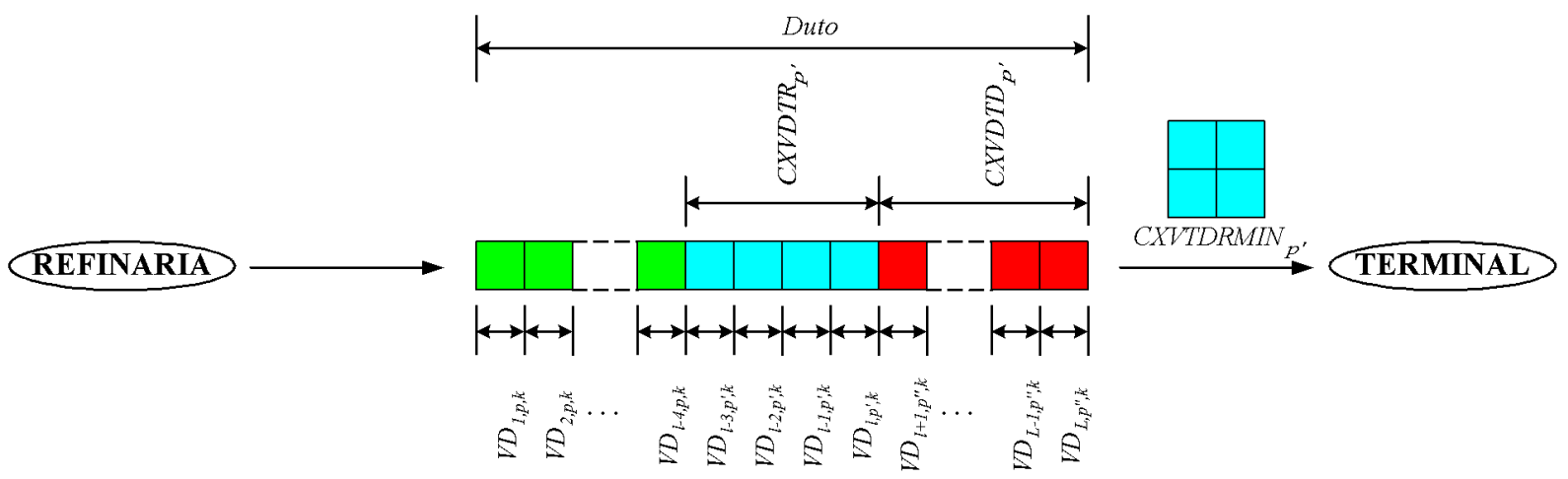

Figura 6.3: llustração da situação 3 para corte completo

Situação 4 - Nesta situação o terminal necessita de uma quantidade do produto $p$, e no instante inicial esta quantidade é maior que a quantidade que está presente dentro do duto. A figura 6.4 ilustra esta situação. 


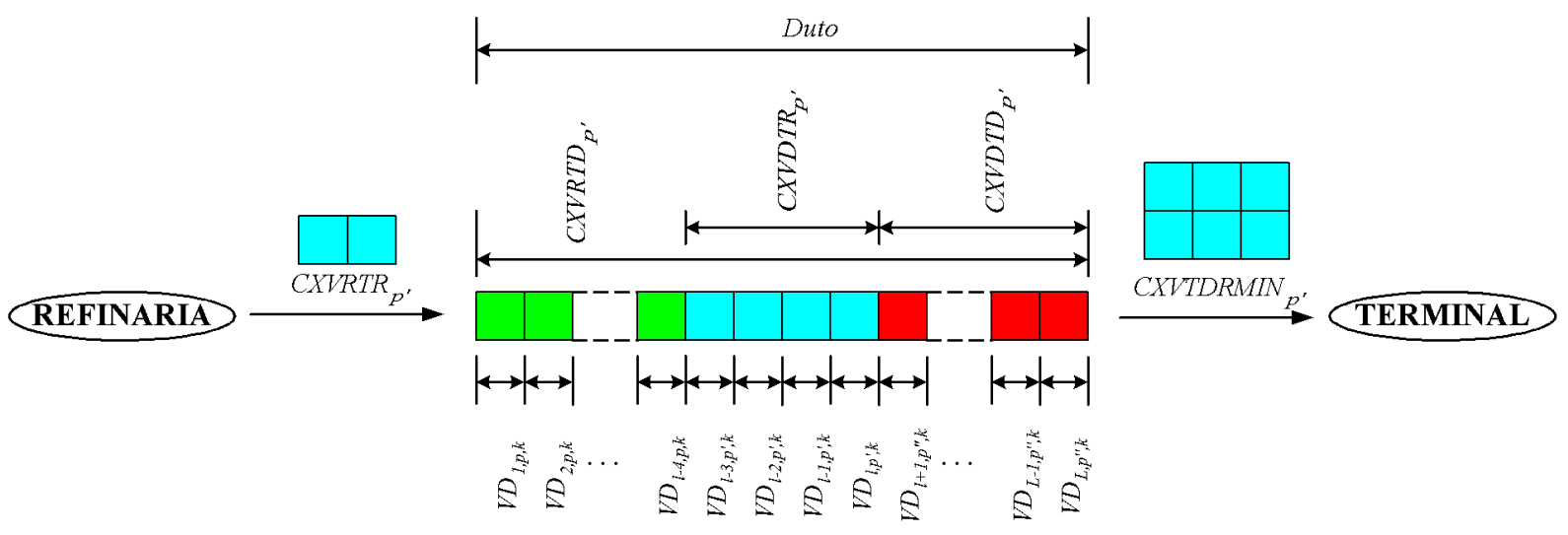

Figura 6.4: llustração da situação 4 para corte completo

Existem as mesmas 4 situações possíveis na movimentação do terminal para a refinaria para cada produto $p$.

A formulação dos parâmetros CXVRDEMIN ${\text { e } C X V T D E M I N_{p}}_{p}$ que indicam a necessidade de enviar da refinaria (eq. 6.9) e do terminal (eq. 6.10) é a seguinte:

$\operatorname{CXVRDEMIN~}_{p}=\max \left\{0,\left\lceil\frac{\sum_{k=1}^{K}\left(C R_{R O R_{p, k}}-C V R O E_{p, k}\right)-\left(C V R M A X_{p}-C V R I_{p}\right)}{C V D L}\right\rceil\right\} \forall p$

$\operatorname{CXVTDEMIN~}_{p}=\max \left\{0,\left\lceil\frac{\sum_{k=1}^{K}\left(\text { CVTOR }_{p, k}-\text { CVTOE }_{p, k}\right)-\left(\text { CVTMAX }_{p}-C V T I_{p}\right)}{C V D L}\right\rceil\right\} \forall$

A formulação dos parâmetros $C X V T D R M I N_{p}$ e $C X V R D R M I N_{p}$ que indicam a necessidade de receber no terminal (eq. 6.11) e na refinaria (eq. 6.12) é a seguinte: 
$C X \operatorname{CTDRMIN}_{p}=\max \left\{0,\left[\frac{\sum_{k=1}^{K}\left(\mathrm{CVTOE}_{p, k}-C V T O R_{p, k}\right)-\left(C V T I_{p}-C V T M I N_{p}\right)}{C V D L}\right]\right\} \forall p$

$C X V \operatorname{RDRMIN}_{p}=\max \left\{0,\left[\frac{\sum_{k=1}^{K}\left(C V R O E_{p, k}-C V R O R_{p, k}\right)-\left(C V R I_{p}-C V R M I N_{p}\right)}{C V D L}\right]\right\} \forall p$

O termo 「 $\rceil$ nas equações (6.9), (6.10), (6.11) e (6.12) representa a função de maior valor inteiro.

De forma semelhante à formulação das equações (6.1) e (6.2), no numerador da equação (6.9) o termo $\sum_{k=1}^{K}\left(C V R O R_{p, k}-C V R O E_{p, k}\right)$ representa a variação de volume do produto $p$ na refinaria ao longo do horizonte de operação (quando positiva, significa maior quantidade de produto produzido/recebido do que saindo da refinaria) e o termo $\left(C V R M A X_{p}-C V R I_{p}\right)$, da mesma equação, representa o volume do produto $p$ para atingir o volume máximo na refinaria a partir do instante inicial. A diferença entre estes dois termos, caso seja positiva, representa o volume mínimo necessário do produto $p$ para não violar o volume máximo na refinaria. De forma análoga são interpretados os termos da equação (6.10).

Na equação (6.11) no numerador o termo $\sum_{k=1}^{K}\left(C V T O E_{p, k}-C V T O R_{p, k}\right)$ representa a variação de volume do produto $p$ no terminal ao longo do horizonte de operação (quando positiva, significa maior quantidade de produto saindo do que entrando no terminal) e o termo $\left(C V T I_{p}-C V T M I N_{p}\right)$ representa o volume do produto $p$ para atingir o limite mínimo no terminal a partir do instante inicial. A diferença entre estes dois termos, caso o valor seja positiva, representa o volume mínimo necessário do produto $p$ para não violar o volume mínimo no terminal. De forma análoga os termos da equação (6.12) é interpretada para a refinaria. 
A proposição lógica (6.13) representa a identificação da situação 2. As equações (6.14) representam os cálculos dos parâmetros relativos à situação 2 .

$$
\begin{aligned}
& \left(C X V \operatorname{TDRMIN}_{p}>0\right) \wedge\left(\sum_{l=1}^{L}\left(C X V D I_{l, p}\right)>0\right) \wedge
\end{aligned}
$$

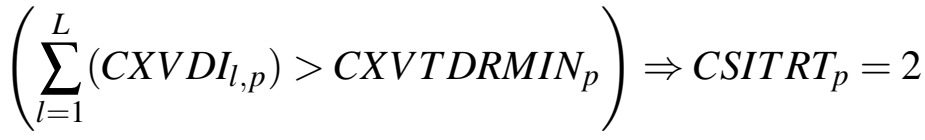

$$
\begin{aligned}
& \forall p \\
& C L M A X_{p}=\max _{l}\left\{l \cdot X V D I_{l, p}\right\} \\
& \forall p \\
& C X V D T D_{p}=\left(L-C L M A X_{p}\right) \\
& \forall p \in\left(C S I T R T_{p}=2\right) \\
& C X V D T R_{p}=C X V T D R M I N_{p} \\
& \forall p \in\left(\operatorname{CSITRT}_{p}=2\right) \\
& C X V D T T D_{p}=C X V D T D_{p}+C X V D T R_{p} \\
& \forall p \in\left(C \operatorname{CSITRT} T_{p}=2\right) \\
& C X V R T D_{p}=0 \\
& \forall p \in\left(C S I T R T_{p}=2\right) \\
& C X V R T R_{p}=0 \\
& \forall p \in\left(\operatorname{CSITRT}_{p}=2\right)
\end{aligned}
$$

A proposição lógica (6.15) representa a identificação da situação 3. As equações (6.16) representam os cálculos dos parâmetros relativos à situação 3.

$$
\begin{array}{ll}
\left(C X V T D R M I N_{p}>0\right) \wedge\left(\sum_{l=1}^{L}\left(C X V D I_{l, p}\right)>0\right) \wedge & \\
\left(\sum_{l=1}^{L}\left(C X V D I_{l, p}\right)=C X V T D R M I N_{p}\right) \Rightarrow C S I T R T_{p}=3 & \forall p \\
C L M A X_{p}=\max _{l}\left\{l \cdot X V D I_{l, p}\right\} & \forall p \\
C X V D T D_{p}=\left(L-C L M A X_{p}\right) & \forall p \in\left(\operatorname{CSITRT}_{p}=3\right) \\
C X V D T R_{p}=C X V T D R M I N_{p} & \forall p \in\left(\operatorname{CSITRT}_{p}=3\right) \\
C X V D T T D_{p}=C X V D T D_{p}+C X V D T R_{p} & \forall p \in\left(\operatorname{CSITRT}_{p}=3\right) \\
C X V R T D_{p}=0 & \forall p \in\left(\operatorname{CSITRT}_{p}=3\right) \\
C X V R T R_{p}=0 & \forall p)
\end{array}
$$


A proposição lógica (6.17) representa a identificação da situação 4. As equações (6.18) representam os cálculos dos parâmetros relativos à situação 4 .

$\left(C X V T \operatorname{DRMIN}_{p}>0\right) \wedge\left(\sum_{l=1}^{L}\left(C X V D I_{l, p}\right)<C X V T D R M I N_{p}\right) \Rightarrow \operatorname{CSITRT}_{p}=4 \quad \forall p$

$$
\begin{array}{ll}
C X V D T D_{p}=0 & \forall p \in\left(\operatorname{CSITRT}_{p}=4\right) \\
C X V D T R_{p}=0 & \forall p \in\left(\operatorname{CSITRT}_{p}=4\right) \\
C X V D T T D_{p}=C X V D T D_{p}+C X V D T R_{p} & \forall p \in\left(C S I T R T_{p}=4\right) \\
C X V R T D_{p}=L & \forall p \in\left(\operatorname{CSITRT}_{p}=4\right) \\
C X V R T R_{p}=C X V T D R M I N_{p}-\sum_{l=1}^{L}\left(C X V D I_{l, p}\right) & \forall p \in\left(\operatorname{CSITRT}_{p}=4\right)
\end{array}
$$

Compondo a situação 1 para todos os produtos, então tem-se a equação (6.19).

$C X V M R D=\sum_{p=1}^{P}\left(C X V R D E M I N_{p}\right)$

Na equação (6.19) o termo $C X V R D E M I N_{p}$ representa a quantidade mínima de lotes necessária do produto $p$ que deve ser enviada da refinaria para o duto. $O$ valor calculado do parâmetro $C X V M R D$ é o somatório por produto das quantidades mínimas de lotes necessárias que devem ser enviadas da refinaria para o terminal.

Compondo as três últimas situações para todos os produtos, então tem-se as equações (6.20) e (6.21).

$$
\begin{aligned}
& C X V M D T=\max _{p}\left\{\max \left(C X V D T T D_{p}, C X V R T D_{p}\right)\right\} \\
& C X V M R T=\sum_{p=1}^{P}\left(C X V R T R_{p}\right)
\end{aligned}
$$

Na equação (6.20) o termo $C X V D T T D_{p}$ representa a quantidade de lotes do duto para o terminal para o produto $p$ caso esteja na situação 2 ou 3; em outros casos o valor deste termo é igual a zero, o termo $C X V R T D_{p}$ representa a quantidade de lotes do duto para o terminal do produto $p$ caso esteja na situação 4; em outros casos o valor deste termo é igual a zero. O valor calculado do parâmetro $C X V M D T$ é o maior 
valor da quantidade de lotes do duto para o terminal considerando todos os produtos e situações.

Na equação (6.21) o termo $C X V R T R_{p}$ representa a quantidade de lotes da refinaria para o terminal para o produto $p$ caso esteja na situação 4; em outros casos o valor deste termo é igual a zero. O valor calculado do parâmetro CXVMRT é somatório das quantidades de lotes da refinaria para o terminal por produto $p$ quando se encontra na situação 4.

A composição de todas as situações resulta na equação (6.22).

$C X M D F M I N=\max \{(C X V M D T+C X V M R T), C X V M R D\}$

Com os parâmetros $C X V M D T, C X V M R T$ e $C X V M R D$ determina-se a quantidade mínima da variável $X M D F$, representada pela equação (6.22).

Para as movimentações do terminal para a refinaria podemos descrever as quatro situações de forma análoga. As figuras 6.5 a 6.8 ilustram as situações 01 a 04 , respectivamente.

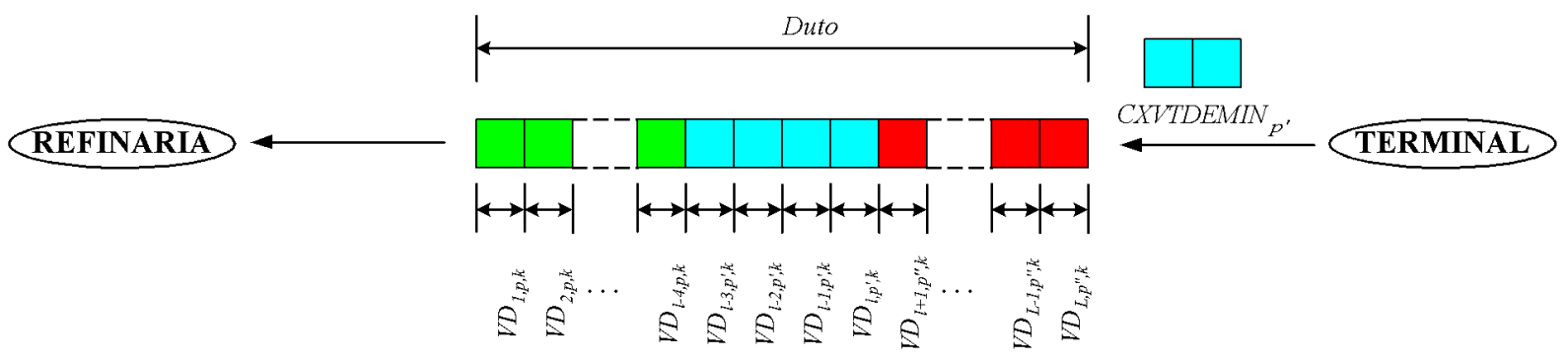

Figura 6.5: Ilustração da situação 1 para corte completo no sentido terminal-refinaria

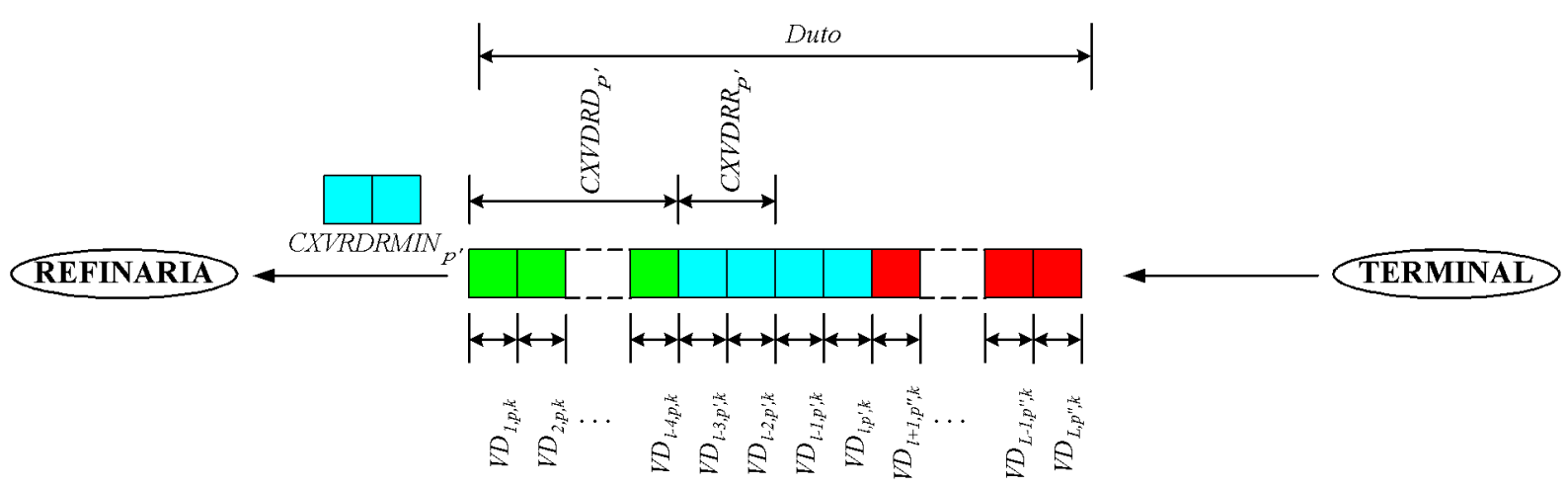

Figura 6.6: Ilustração da situação 2 para corte completo no sentido terminal-refinaria 


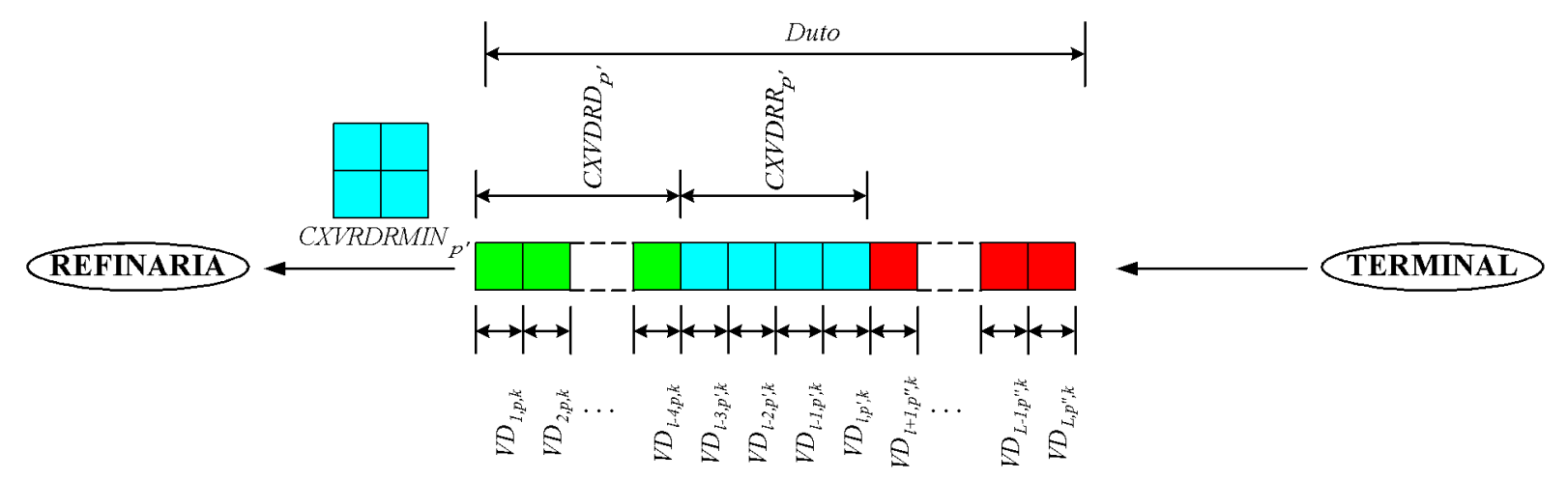

Figura 6.7: Ilustração da situação 3 para corte completo no sentido terminal-refinaria

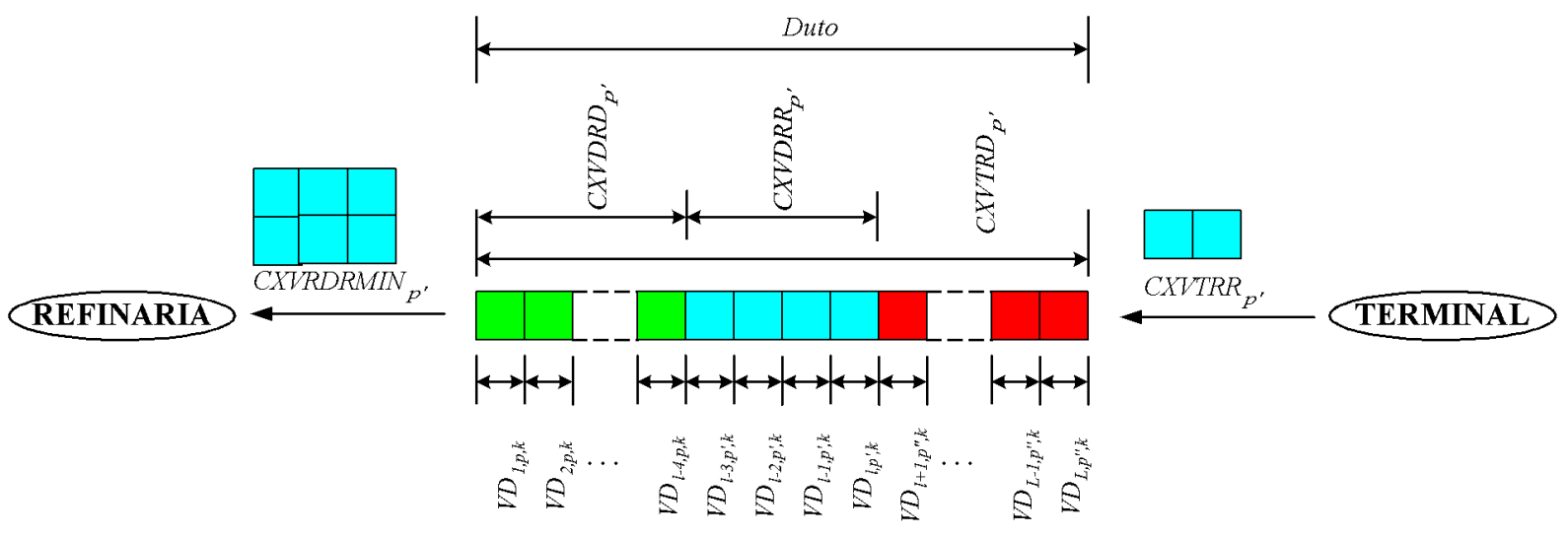

Figura 6.8: Ilustração da situação 4 para corte completo no sentido terminal-refinaria 
A proposição lógica (6.23) representa a identificação da situação 2. As equações (6.24) representam os cálculos dos parâmetros relativos à situação 2 .

$$
\begin{aligned}
& \left(C X V \operatorname{RDRMIN}_{p}>0\right) \wedge\left(\sum_{l=1}^{L}\left(C X V D I_{l, p}\right)>0\right) \wedge \\
& \left(\sum_{l=1}^{L}\left(C X V D I_{l, p}\right)>C X V R D R M I N_{p}\right) \Rightarrow \operatorname{CSITTR}_{p}=2
\end{aligned}
$$

$\operatorname{CLMIN}_{p}=\min _{l}\left\{l \cdot X V D I_{l, p}\right\}$

$\forall p$

$C X V D R D_{p}=\left(C L M I N_{p}-1\right)$

$\forall p \in\left(\operatorname{CSITTR}_{p}=2\right)$

$C X V D R R_{p}=C X V R D R M I N_{p}$ $\forall p \in\left(\operatorname{CSITTR}_{p}=2\right)$

$C X V D R T D_{p}=C X V D R D_{p}+C X V D R R_{p}$ $\forall p \in\left(\operatorname{CSITTR}_{p}=2\right)$

$C X V T R D_{p}=0$ $\forall p \in\left(\operatorname{CSITTR}_{p}=2\right)$

$C X V T R R_{p}=0$ $\forall p \in\left(\operatorname{CSITTR}_{p}=2\right)$

A proposição lógica (6.25) representa a identificação da situação 3. As equações (6.26) representam os cálculos dos parâmetros relativos à situação 3 .

$$
\begin{gathered}
\left(C X V \operatorname{RDRMIN}_{p}>0\right) \wedge\left(\sum_{l=1}^{L}\left(C X V D I_{l, p}\right)>0\right) \wedge \\
\left(\sum_{l=1}^{L}\left(C X V D I_{l, p}\right)=C X V \operatorname{RDRMIN}_{p}\right) \Rightarrow \operatorname{CSITTR}_{p}=3
\end{gathered}
$$




$$
\begin{aligned}
& \operatorname{CLMIN}_{p}=\min _{l}\left\{l \cdot X V D I_{l, p}\right\} \quad \forall p \\
& C X V \operatorname{DRD}_{p}=\left(\operatorname{CLMIN}_{p}-1\right) \quad \forall p \in\left(\operatorname{CSITTR}_{p}=3\right) \\
& C X V D R R_{p}=C X V R D R M I N_{p} \quad \forall p \in\left(\operatorname{CSITTR}_{p}=3\right) \\
& C X V D R T D_{p}=C X V D R D_{p}+C X V D R R_{p} \quad \forall p \in\left(\operatorname{CSITTR}_{p}=3\right) \\
& C X V \operatorname{TRD}_{p}=0 \quad \forall p \in\left(\operatorname{CSITTR}_{p}=3\right) \\
& C X V T R R_{p}=0 \quad \forall p \in\left(\operatorname{CSITTR}_{p}=3\right)
\end{aligned}
$$

A proposição lógica (6.27) representa a identificação da situação 4. As equações (6.28) representam os cálculos dos parâmetros relativos à situação 4 .

$\left(\operatorname{CXVRDRMIN}_{p}>0\right) \wedge\left(\sum_{l=1}^{L}\left(C X V D I_{l, p}\right)<C X V \operatorname{RDRMIN}_{p}\right) \Rightarrow \operatorname{CSITTR}_{p}=4 \quad \forall p$

$$
\begin{array}{ll}
C X V D R D_{p}=0 & \forall p \in\left(\operatorname{CSITTR}_{p}=4\right) \\
C X V D R R_{p}=0 & \forall p \in\left(\operatorname{CSITTR}_{p}=4\right) \\
C X V D R T D_{p}=C X V D R D_{p}+C X V D R R_{p} & \forall p \in\left(\operatorname{CSITTR}_{p}=4\right) \\
C X V T R D_{p}=L & \forall p \in\left(\operatorname{CSITTR}_{p}=4\right) \\
C X V T R R_{p}=C X V R D R M I N_{p}-\sum_{l=1}^{L}\left(C X V D I_{l, p}\right) & \forall p \in\left(\operatorname{CSITTR}_{p}=4\right)
\end{array}
$$

Compondo a situação 1 para todos os produtos, então tem-se a equação (6.29).

$C X V M T D=\sum_{p=1}^{P}\left(C X V T D E M I N_{p}\right)$

$\mathrm{Na}$ equação (6.29) o termo $C X V T D E M I N_{p}$ representa a quantidade mínima de lotes necessária do produto $p$ que deve ser enviada do terminal para o duto. $O$ valor calculado do parâmetro $C X V M T D$ é o somatório por produto das quantidades mínimas de lotes necessárias que devem ser enviadas do terminal para a refinaria. 
Compondo as três últimas situações para todos os produtos, então tem-se as equações (6.30) e (6.31).

$$
\begin{aligned}
& C X V M D R=\max _{p}\left\{\max \left(C X V D R T D_{p}, C X V T R D_{p}\right)\right\} \\
& C X V M T R=\sum_{p=1}^{P}\left(C X V T R R_{p}\right)
\end{aligned}
$$

Na equação (6.30) o termo $C X V D R T D_{p}$ representa a quantidade de lotes do duto para a refinaria para o produto $p$ caso esteja na situação 2 ou 3 (em outros casos o valor deste termo é igual a zero) e o termo $C X V T R D_{p}$ representa a quantidade de lotes do duto para a refinaria do produto $p$ caso esteja na situação 4 (em outros casos o valor deste termo é igual a zero). O valor calculado do parâmetro $C X V M D R$ é o maior valor da quantidade de lotes do duto para a refinaria considerando todos os produtos e situações.

Na equação (6.31) o termo $C X V T R R_{p}$ representa a quantidade de lotes do terminal para a refinaria do produto $p$ caso esteja na situação 4 (em outros casos o valor deste termo é igual a zero). O valor calculado do parâmetro $C X V M T R$ é somatório das quantidades de lotes do terminal para a refinaria por produto $p$ quando se encontra na situação 4.

A composição de todas as situações tem-se a equação (6.32).

$C X M D R M I N=\max \{(C X V M D R+C X V M T R), C X V M T D\}$

Com os parâmetros $C X V M D R, C X V M T R$ e $C X V M T D$ determina-se a quantidade mínima da variável $X M D R$, representada pela equação (6.32).

Então como limites das quantidades mínimas a serem bombeadas podem-se definir as restrições (6.33) e (6.34). Podem-se também definir quantidades mínimas a serem bombeadas de cada produto pelas restrições (6.35) e (6.36). 


$$
\begin{aligned}
& \sum_{k=1}^{K}\left(X M D F_{k}\right) \geq C X M D F M I N \\
& \sum_{k=1}^{K}\left(X M D R_{k}\right) \geq C X M D R M I N \\
& \sum_{k=1}^{K}\left(X V R D E_{p, k}\right) \geq \max \left\{C X V R T R_{p}, C X V R D E M I N_{p}\right\} \\
& \forall p \in\left\{\left(C X V R T R_{p}>0\right) \vee\left(C X V \operatorname{RDEMIN}_{p}>0\right)\right\} \\
& \sum_{k=1}^{K}\left(X V T D E_{p, k}\right) \geq \max \left\{C X V T R R_{p}, C X V T D E M I N_{p}\right\} \\
& \forall p \in\left\{\left(C X V T R R_{p}>0\right) \vee\left(C X V T D E M I N_{p}>0\right)\right\}
\end{aligned}
$$


Portanto o modelo proposto (MCC) nesta variação é da seguinte forma:

MCC:

Minimizar (4.40)

sujeito a:

(4.3), (4.4), (4.8) e (4.9) restrições de armazenamento da refinaria

(4.6) e (4.7) equações de troca entre refinaria e duto

(4.10), (4.11), (4.15) e (4.16) restrições de armazenamento no terminal

(4.13) e (4.14) equações de troca entre terminal e duto

(4.17), (4.31), (4.32), (4.33), (4.34) e (4.35) restrições que representam os estados do duto

(4.36), (4.37), (4.38) e (4.39) restrições de interface de produtos dentro do duto

(6.33) e (6.34) limite da quantidade mínima a ser bombeada

(6.35) e (6.36) limite da quantidade mínima de cada produto a ser bombeada

$$
\begin{aligned}
& \left(X V R D E_{p, k}, X V T D E_{p, k}\right) \in\{0 ; 1\} \\
& \left(X M D F_{k}, X M D R_{k}, X M D N_{k}\right) \in[0 ; 1] \\
& \left(X V R D R_{p, k}, X V T D R_{p, k}, X V D_{l, p, k}\right) \in[0 ; 1] \\
& \left(X I P R D_{p, p^{\prime}, k}, X I P T D_{p, p^{\prime}, k}\right) \in[0 ; 1] \\
& \left(V R_{p, k}, V R D R_{p, k}, V R D E_{p, k}, V T_{p, k}, V T D R_{p, k}, V T D E_{p, k}, V D_{l, p, k}, C U S T O\right) \geq 0
\end{aligned}
$$

\subsubsection{EXEMPLOS ILUSTRATIVOS DOS CORTES VÁLIDOS}

Nesta seção são apresentados exemplos para elucidação da formulação dos cortes válidos. São apresentados 2 exemplos:

- Exemplo 1 - Este exemplo ilustra a situação 1 e a situação 4 para o produto $p=1$ e a situação 2 para o produto $p=2$. Não são considerados neste exemplo 
os cortes relativos às movimentações no sentido reverso do duto (terminal $\rightarrow$ refinaria). A figura 6.9 ilustra este exemplo;

- Exemplo 2 - Este exemplo ilustra a situação 1 e a situação 4 para o produto $p=1$, a situação 4 para o produto $p=2$ e a situação 1 para o produto $p=3$. Não são considerados neste exemplo os cortes relativos às movimentações no sentido reverso do duto (terminal $\rightarrow$ refinaria). A figura 6.10 ilustra este exemplo.

Para todos os exemplos o duto se encontra cheio do produto $p=2\left(X V D I_{l, 2}=\right.$ $1 \forall l)$ e o duto está dividido em 6 lotes $(L=6)$. Se o exemplo não citar os valores dos parâmetros calculados, $C X V R D E M I N_{p}$ e $C X V T R M I N_{p}$, então o valor destes parâmetros é igual a zero.

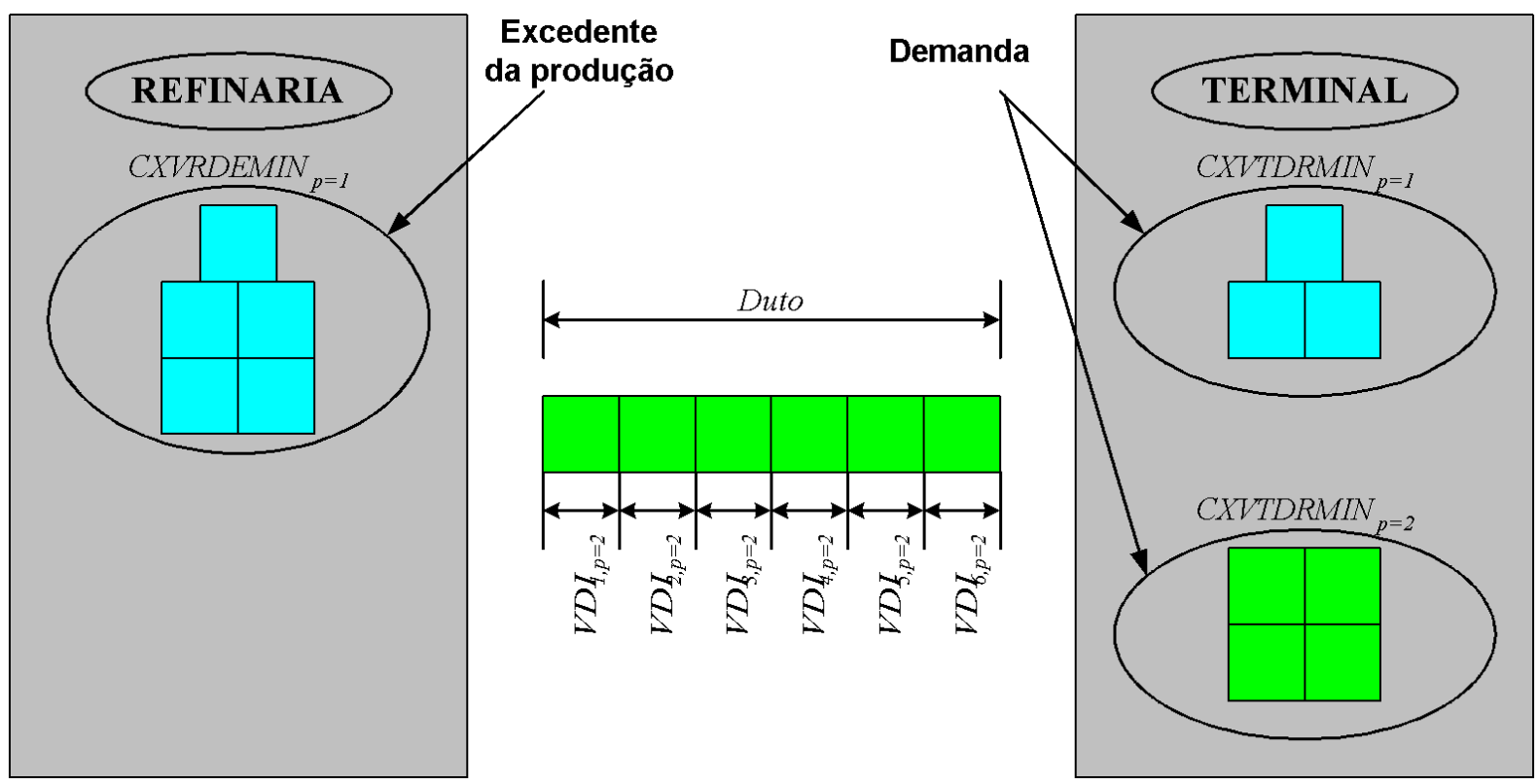

Figura 6.9: Ilustração do exemplo 1 


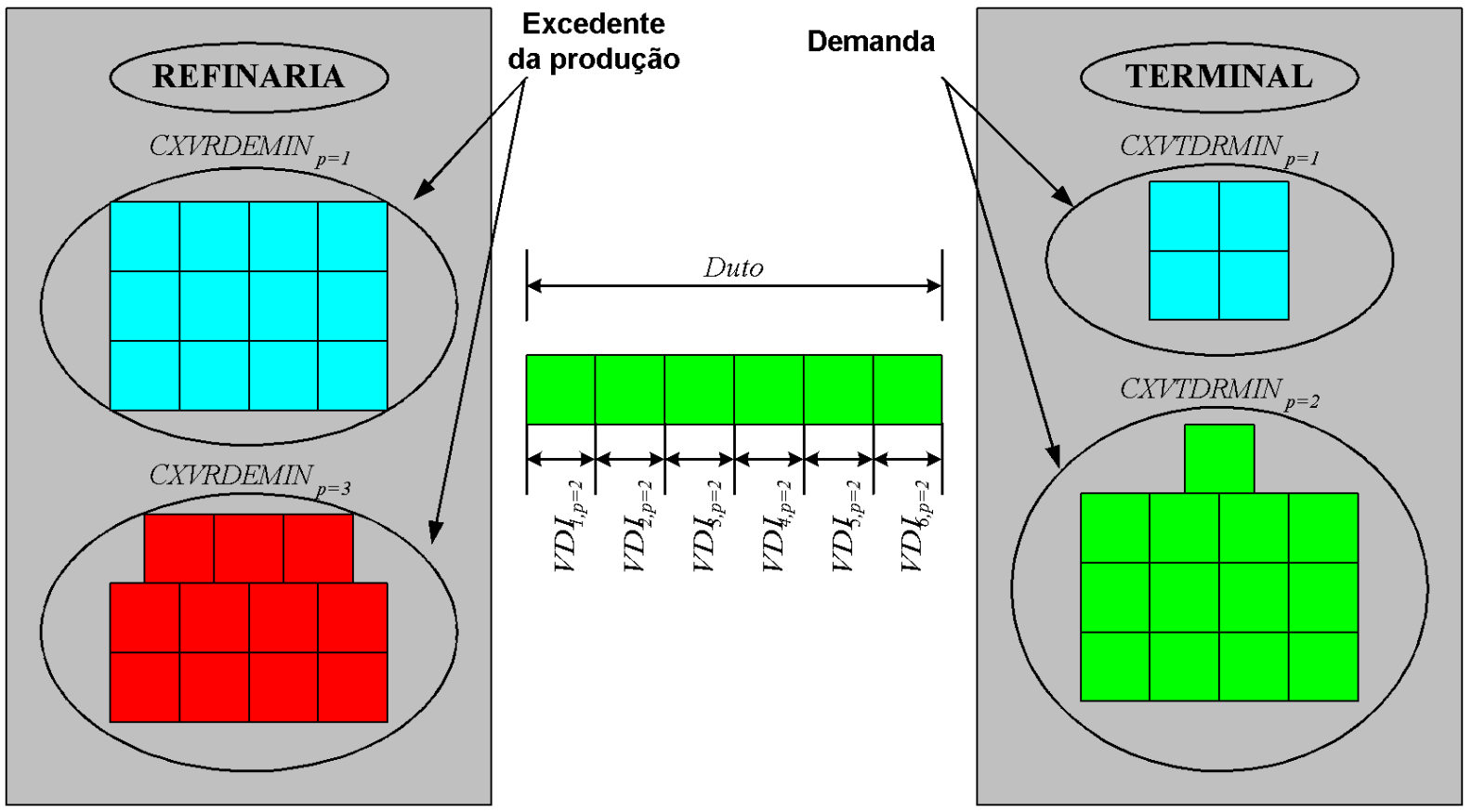

Figura 6.10: Ilustração do exemplo 2

Exemplo 1 - O parâmetro calculado $C X V R D E M I N_{1}=5$ indica a situação 1 para o produto $p=1$, o parâmetro $C X V T D R M I N_{1}=3$ indica a situação 4 para o produto $p=1$ e o parâmetro calculado $C X V T D R M I N_{2}=4$ indica a situação 2 para o produto $p=2$.

Para o produto $p=1$ a proposição lógica (6.17) é válida e as equações (6.18) são as seguintes:

$$
\begin{aligned}
& C X V D T D_{1}=0 \\
& C X V D T R_{1}=0 \\
& C X V D T T D_{1}=C X V D T D_{1}+C X V D T R_{1}=0 \\
& C X V R T D_{1}=L=6 \\
& C X V R T R_{1}=C X V T D R M I N_{1}-\sum_{l=1}^{L}\left(C X V D I_{l, 1}\right)=3-0=3
\end{aligned}
$$


Para o produto $p=2$ a proposição lógica (6.13) é válida e as equações (6.14) são as seguintes:

$$
\begin{aligned}
& C L M A X_{2}=\max _{l}\left\{l \cdot X V D I_{l, 2}\right\}=6 \\
& C X V D T D_{2}=\left(L-C L M A X_{2}\right)=(6-6)=0 \\
& C X V D T R_{2}=C X V T D R M I N_{2}=4 \\
& C X V D T T D_{2}=C X V D T D_{2}+C X V D T R_{2}=0+4=4 \\
& C X V R T D_{2}=0 \\
& C X V R T R_{2}=0
\end{aligned}
$$

Portanto as equações (6.19), (6.20), (6.21) e (6.22) que combinam as situações são as seguintes:

$$
\begin{aligned}
& \begin{aligned}
C X V M R D & =\sum_{p=1}^{P}\left(C X V R D E M I N_{p}\right)=(5)_{p=1}+(0)_{p=2}=5
\end{aligned} \\
& \begin{aligned}
C X V M D T & =\max _{p}\left\{\max \left(C X V D T T D_{p}, C X V R T D_{p}\right)\right\}=\max _{p}\left\{(\max (0,6))_{p=1},(\max (4,0))_{p=2}\right\} \\
& =6
\end{aligned} \\
& \begin{aligned}
C X V M R T & =\sum_{p=1}^{P}\left(C X V R T R_{p}\right)=(3)_{p=1}+(0)_{p=2}=3
\end{aligned} \\
& C X M D F M I N=\max \{(C X V M D T+C X V M R T), C X V M R D\}=\max \{(6+3), 5\}=9
\end{aligned}
$$

Como os parâmetros $C X V R D E M I N_{1}=5, C X V R T R_{1}=3$ e $C X M D F M I N=9$, então as restrições (6.34) e (6.36) não são válidas e as restrições (6.33), (6.35) são as seguintes:

$$
\begin{aligned}
& \sum_{k=1}^{K}\left(X M D F_{k}\right) \geq C X M D F M I N=9 \\
& \sum_{k=1}^{K}\left(X V R D E_{1, k}\right) \geq \max \left\{C X V R T R_{1}, C X V R D E M I N_{1}\right\}=\max \{3,5\}=5
\end{aligned}
$$

Portanto neste caso interpretando as restrições: o duto deve movimentar ao menos 9 lotes no sentido para frente $\left(\sum_{k=1}^{K}\left(X M D F_{k}\right) \geq 9\right)$ e a refinaria deve enviar ao menos 5 lotes para o terminal do produto $p=1\left(\sum_{k=1}^{K}\left(X V R D E_{1, k}\right) \geq 5\right)$.

Exemplo 2 - O parâmetro calculado $C X V R D E M I N_{1}=12$ indica a situação 1 para o produto $p=1$, o parâmetro calculado $C X V T D R M I N_{1}=4$ indica a situação 4 para o 
produto $p=1$, o parâmetro calculado $C X V T D R M I N_{2}=13$ indica a situação 4 para o produto $p=2$ e o parâmetro calculado $C X V R D E M I N_{3}=11$ indica a situação 1 para o produto $p=3$.

Para o produto $p=1$ a proposição lógica (6.17) é válida e as equações (6.18) são as seguintes:

$C X V D T D_{1}=0$

$C X V D T R_{1}=0$

$C X V D T T D_{1}=C X V D T D_{1}+C X V D T R_{1}=0$

$C X V R T D_{1}=L=6$

$C X V R T R_{1}=C X V T D R M I N_{1}-\sum_{l=1}^{L}\left(C X V D I_{l, 1}\right)=4-0=4$

Para o produto $p=2$ a proposição lógica (6.17) é válida e as equações (6.18) são as seguintes:

$C X V D T D_{2}=0$

$C X V D T R_{2}=0$

$C X V D T T D_{2}=C X V D T D_{1}+C X V D T R_{1}=0$

$C X V R T D_{2}=L=6$

$C X V R T R_{2}=X C V T D R M I N_{2}-\sum_{l=1}^{L}\left(C X V D I_{l, 2}\right)=13-6=7$ 
Portanto as equações (6.19), (6.20), (6.21) e (6.22) que combinam as situações são as seguintes:

$$
\begin{aligned}
& \begin{aligned}
C X V M R D & =\sum_{p=1}^{P}\left(C X V R D E M I N_{p}\right)=(12)_{p=1}+(0)_{p=2}+(11)_{p=3}=23 \\
C X V M D T & =\max _{p}\left\{\max \left(C X V D T T D_{p}, C X V R T D_{p}\right)\right\} \\
& =\max _{p}\left\{(\max (0,6))_{p=1},(\max (0,6))_{p=2},\left(\max (0,0)_{p=3}\right)\right\} \\
& =\max _{p}\left\{(6)_{p=1},(6)_{p=2},(0)_{p=3}\right\}=6
\end{aligned} \\
& C X V M R T=\sum_{p=1}^{P}\left(C X V R T R_{p}\right)=\left((4)_{p=1}+(7)_{p=2}+(0)_{p=3}\right)=11 \\
& C X M D F M I N=\max \{(C X V M D T+C X V M R T), C X V M R D\}=\max \{(6+11), 23\} \\
& =\max \{17,23\}=23
\end{aligned}
$$

Como os parâmetros $C X V R D E M I N_{1}=12, C X V R T R_{1}=4, C X V R T R_{2}=7, C X V R D E M I N_{3}=$ 11 e $C X M D F M I N=23$, então as restrições (6.34) e (6.36) não são válidas e as restrições (6.33), (6.35) são as seguintes:

$\sum_{k=1}^{K}\left(X M D F_{k}\right) \geq C X M D F M I N=23$

$\sum_{k=1}^{K}\left(X V R D E_{1, k}\right) \geq \max \left\{C X V R T R_{1}, C X V R D E M I N_{1}\right\}=\max \{4,12\}=12$

$\sum_{k=1}^{K}\left(X V R D E_{2, k}\right) \geq \max \left\{C X V R T R_{2}, C X V R D E M I N_{2}\right\}=\max \{7,0\}=7$

$\sum_{k=1}^{K}\left(X V R D E_{3, k}\right) \geq \max \left\{C X V R T R_{3}, C X V R D E M I N_{3}\right\}=\max \{0,11\}=11$

Portanto neste caso as restrições impõem o seguinte: o duto deve movimentar ao menos 25 lotes no sentido para frente $\left(\sum_{k=1}^{K}\left(X M D F_{k}\right) \geq 23\right)$, a refinaria deve enviar ao menos 12 lotes para o terminal do produto $p=1\left(\sum_{k=1}^{K}\left(X V R D E_{1, k}\right) \geq 12\right)$, a refinaria deve enviar ao menos 7 lotes para o terminal do produto $p=2\left(\sum_{k=1}^{K}\left(X V R D E_{2, k}\right) \geq 7\right)$ e a refinaria deve enviar ao menos 11 lotes para o terminal do produto $p=3\left(\sum_{k=1}^{K}\left(X V R D E_{3, k}\right) \geq\right.$ 11). 
Finalmente a equação (4.34) impõe neste caso que:

$$
\begin{aligned}
\sum_{k=1}^{K}\left(X M D F_{k}\right) & =\sum_{p=1}^{P} \sum_{k=1}^{K}\left(X V R D E_{p, k}\right) \\
& =\underbrace{\left[\sum_{k=1}^{K}\left(X V R D E_{p, k}\right)\right]_{p=1}}_{\geq 12}+\underbrace{\left[\sum_{k=1}^{K}\left(X V R D E_{p, k}\right)\right]_{p=2}}_{\geq 7}+\underbrace{\left[\sum_{k=1}^{K}\left(X V R D E_{p, k}\right)\right]_{p=3}}_{\geq 11} \\
& \therefore \\
\sum_{k=1}^{K}\left(X M D F_{k}\right) & \geq 30
\end{aligned}
$$

Então as inequações (6.35) representam cortes distintos da inequação (6.33) e não redundantes.

\subsubsection{RESTRIÇÕES DE CORTE POR INSTANTE DE TEMPO}

Uma adaptação possível na modelagem apresentada por Rejowski Jr e Pinto (2004), que equivale as equações (6.9) a (6.36), é ao invés de gerar um limite da quantidade mínima a ser bombeada para todo o período, definir um limite da quantidade mínima a ser bombeada para cada instante $k$.

Os parâmetros para esta adaptação são apresentados na tabela 6.4, a qual apresenta a identificação e a descrição de cada um deste.

Tabela 6.4: Parâmetros associadas aos cortes completos por tempo

\begin{tabular}{|l|l|}
\hline \multicolumn{1}{|c|}{ Parâmetro } & \multicolumn{1}{c|}{ Descrição } \\
\hline CXVRDEMIN & \multicolumn{1}{c|}{$\begin{array}{l}\text { Quantidade mínima de lotes do produto } p \text { que a refinaria deve } \\
\text { enviar para o duto até o instante } k .\end{array}$} \\
\hline$C X V T D R M I N_{p, k}$ & $\begin{array}{l}\text { Quantidade mínima de lotes do produto } p \text { que o terminal deve } \\
\text { receber do duto até o instante } k .\end{array}$ \\
\hline$C X V D T D_{p, k}$ & $\begin{array}{l}\text { Quantidade de lotes para iniciar o recebimento do produto } p \text { no } \\
\text { terminal que tem como origem o duto no instante } k .\end{array}$ \\
\hline
\end{tabular}




\begin{tabular}{|c|c|}
\hline Parâmetro & Descrição \\
\hline$C X V D T R_{p, k}$ & $\begin{array}{l}\text { Quantidade de lotes do produto } p \text { a ser recebida no terminal ori- } \\
\text { ginada do duto no instante } k \text {. }\end{array}$ \\
\hline$C X V D T T D_{p, k}$ & $\begin{array}{l}\text { Quantidade total de lotes para movimentar o produto } p \text { a ser re- } \\
\text { cebida no terminal originada do duto no instante } k \text {. }\end{array}$ \\
\hline$C X V R T D_{p, k}$ & $\begin{array}{l}\text { Quantidade de lotes para iniciar o recebimento do produto } p \text { no } \\
\text { terminal que tem como origem a refinaria no instante } k \text {. }\end{array}$ \\
\hline$C X V R T R_{p, k}$ & $\begin{array}{l}\text { Quantidade de lotes do produto } p \text { a ser recebida no terminal ori- } \\
\text { ginada na refinaria no instante } k \text {. }\end{array}$ \\
\hline$C X V T D E M I N_{p, k}$ & $\begin{array}{l}\text { Quantidade mínima de lotes do produto } p \text { que o terminal deve } \\
\text { enviar para o duto até o instante } k \text {. }\end{array}$ \\
\hline$C X V R D R M I N_{p, k}$ & $\begin{array}{l}\text { Quantidade mínima de lotes do produto } p \text { que a refinaria deve } \\
\text { receber do duto até o instante } k \text {. }\end{array}$ \\
\hline$C X V D R D_{p, k}$ & $\begin{array}{l}\text { Quantidade de lotes para iniciar o recebimento do produto } p \text { na } \\
\text { refinaria que tem como origem o duto no instante } k \text {. }\end{array}$ \\
\hline$C X V D R R_{p, k}$ & $\begin{array}{l}\text { Quantidade de lotes do produto } p \text { a ser recebida na refinaria ori- } \\
\text { ginada do duto no instante } k \text {. }\end{array}$ \\
\hline$C X V D R T D_{p, k}$ & $\begin{array}{l}\text { Quantidade total de lotes para movimentar o produto } p \text { a ser re- } \\
\text { cebida na refinaria originada do duto no instante } k \text {. }\end{array}$ \\
\hline$C X V T R D_{p, k}$ & $\begin{array}{l}\text { Quantidade de lotes para iniciar o recebimento do produto } p \text { na } \\
\text { refinaria que tem como origem o terminal no instante } k \text {. }\end{array}$ \\
\hline$C X V T R R_{p, k}$ & $\begin{array}{l}\text { Quantidade de lotes do produto } p \text { a ser recebida na refinaria ori- } \\
\text { ginada no terminal no instante } k \text {. }\end{array}$ \\
\hline$C X V M R D_{k}$ & $\begin{array}{l}\text { Quantidade de lotes para movimentar os produtos da refinaria } \\
\text { para dentro do duto no instante } k \text {. }\end{array}$ \\
\hline$C X V M D T_{k}$ & $\begin{array}{l}\text { Quantidade de lotes para movimentar os produtos contidos no } \\
\text { duto para o terminal no instante } k \text {. }\end{array}$ \\
\hline
\end{tabular}




\begin{tabular}{|l|l|}
\hline \multicolumn{1}{|c|}{ Parâmetro } & \multicolumn{1}{c|}{ Descrição } \\
\hline$C X V M R T_{k}$ & $\begin{array}{l}\text { Quantidade de lotes para movimentar os produtos da refinaria } \\
\text { para o terminal no instante } k .\end{array}$ \\
\hline$C X V M T D_{k}$ & $\begin{array}{l}\text { Quantidade de lotes para movimentar os produtos do terminal } \\
\text { para dentro do duto no instante } k .\end{array}$ \\
\hline$C X V M D R_{k}$ & $\begin{array}{l}\text { Quantidade de lotes para movimentar os produtos contidos no } \\
\text { duto para a refinaria no instante } k .\end{array}$ \\
\hline$C X V M T R_{k}$ & $\begin{array}{l}\text { Quantidade de lotes para movimentar os produtos do terminal } \\
\text { para a refinaria no instante } k .\end{array}$ \\
\hline$C S I T R T_{p, k}$ & $\begin{array}{l}\text { Identificação de uma situação para o produto } p \text { na movimentação } \\
\text { entre a refinaria e o terminal no instante } k .\end{array}$ \\
\hline$C S I T T R_{p, k}$ & $\begin{array}{l}\text { Identificação de uma situação para o produto } p \text { na movimentação } \\
\text { entre o terminal e a refinaria no instante } k .\end{array}$ \\
\hline
\end{tabular}

Os parâmetros $C X V R D E M I N_{p, k}$ e $C X V T D E M I N_{p, k}$ são calculados conforme as equações (6.37) e (6.38).

$C X V R D E M I N_{p, k}=$

$$
\max \left\{0,\left[\frac{\sum_{k^{\prime}=1}^{k}\left(C V R O R_{p, k^{\prime}}-C V R O E_{p, k^{\prime}}\right)-\left(C V R M A X_{p}-C V R I_{p}\right)}{C V D L}\right]\right\} \forall p, \forall k
$$

$\operatorname{CXVTDEMIN}_{p, k}=$

$$
\max \left\{0,\left[\frac{\sum_{k^{\prime}=1}^{k}\left(\mathrm{CVTOR}_{p, k^{\prime}}-C V T O E_{p, k^{\prime}}\right)-\left(C V T M A X_{p}-C V T I_{p}\right)}{C V D L}\right]\right\} \forall p, \forall k
$$

Similarmente os parâmetros $C X V T D R M I N_{p, k}$ e $C X V R D R M I N_{p, k}$ são calculados con- 
forme as equações (6.39) e (6.40).

$\operatorname{CXVTDRMIN}_{p, k}=$

$$
\max \left\{0,\left[\frac{\sum_{k^{\prime}=1}^{k}\left(\mathrm{CVTOE}_{p, k^{\prime}}-C V T O R_{p, k^{\prime}}\right)-\left(C V T I_{p}-C V T M I N_{p}\right)}{C V D L}\right\rceil \forall p, \forall k\right.
$$

$C X \operatorname{VDDRMIN}{ }_{p, k}=$

$$
\max \left\{0,\left[\frac{\sum_{k^{\prime}=1}^{k}\left(C V R O E_{p, k^{\prime}}-C V R O R_{p, k^{\prime}}\right)-\left(C V R I_{p}-C V R M I N_{p}\right)}{C V D L}\right]\right\} \forall p, \forall k
$$

Para as movimentações da refinaria para o terminal a proposição lógica (6.41) representa a identificação da situação 2. As equações (6.42) representam os cálculos dos parâmetros relativos à situação 2 .

$$
\begin{aligned}
& \left(C X V T \operatorname{DRMIN}_{p, k}>0\right) \wedge\left(\sum_{l=1}^{L}\left(C X V D I_{l, p}\right)>0\right) \wedge
\end{aligned}
$$

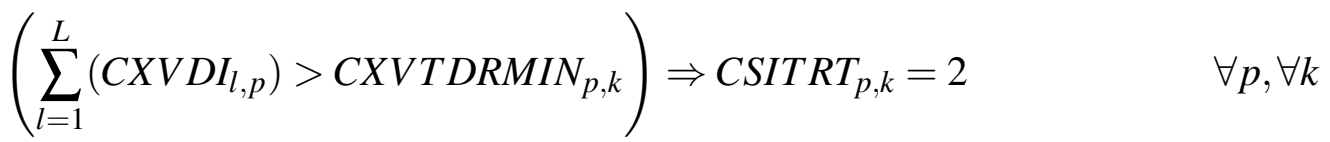

$$
\begin{aligned}
& C L M A X_{p}=\max _{l}\left\{l \cdot X V D I_{l, p}\right\} \quad \forall p \\
& C X V D T D_{p, k}=\left(L-C L M A X_{p}\right) \quad(\forall p, \forall k) \in\left(\operatorname{CSITRT}_{p, k}=2\right) \\
& \mathrm{CXVDTR}_{p, k}=C X V T D R M I N_{p, k} \quad(\forall p, \forall k) \in\left(\operatorname{CSITRT}_{p, k}=2\right) \\
& C X V D T T D_{p, k}=C X V D T D_{p, k}+C X V D T R_{p, k} \quad(\forall p, \forall k) \in\left(C S I T R T_{p, k}=2\right) \\
& C X V R T D_{p, k}=0 \quad(\forall p, \forall k \quad) \in\left(\operatorname{CSITRT}_{p, k}=2\right)(6.42 \mathrm{e}) \\
& C X V R T R_{p, k}=0 \quad(\forall p, \forall k \quad) \in\left(\operatorname{CSITRT}_{p, k}=2\right)
\end{aligned}
$$

Para as movimentações da refinaria para o terminal a proposição lógica (6.43) representa a identificação da situação 3. As equações (6.44) representam os cálculos 
dos parâmetros relativos à situação 3 .

$$
\begin{aligned}
\left(\operatorname{CXVTDRMIN}_{p, k}>0\right) & \wedge\left(\sum_{l=1}^{L}\left(\operatorname{CXVDI}_{l, p}\right)>0\right) \wedge \\
\left(\sum_{l=1}^{L}\left(C X V D I_{l, p}\right)\right. & \left.=C X V T \operatorname{TRMIN}_{p, k}\right) \Rightarrow \operatorname{CSITRT}_{p, k}=3 \quad \forall p, \forall k
\end{aligned}
$$

CLMAX $_{p}=\max _{l}\left\{l \cdot X V D I_{l, p}\right\} \quad \forall p$

$C X V D T D_{p, k}=\left(L-C L M A X_{p}\right) \quad(\forall p, \forall k) \in\left(\operatorname{CSITRT}_{p, k}=3\right)$

$C X V D T R_{p, k}=C X V T D R M I N_{p, k}$

$(\forall p, \forall k) \in\left(\operatorname{CSITRT}_{p, k}=3\right)$

$C X V D T T D_{p, k}=C X V D T D_{p, k}+C X V D T R_{p, k}$

$(\forall p, \forall k) \in\left(\operatorname{CSITRT}_{p, k}=3\right)$

$C X V R T D_{p, k}=0$

$(\forall p, \forall k) \in\left(\operatorname{CSITRT} T_{p, k}=3\right)$

$C X V R T R_{p, k}=0$

$(\forall p, \forall k) \in\left(\operatorname{CSITRT}_{p, k}=3\right)$

Para as movimentações da refinaria para o terminal a proposição lógica (6.45) representa a identificação da situação 4. As equações (6.46) representam os cálculos dos parâmetros relativos à situação 4 .

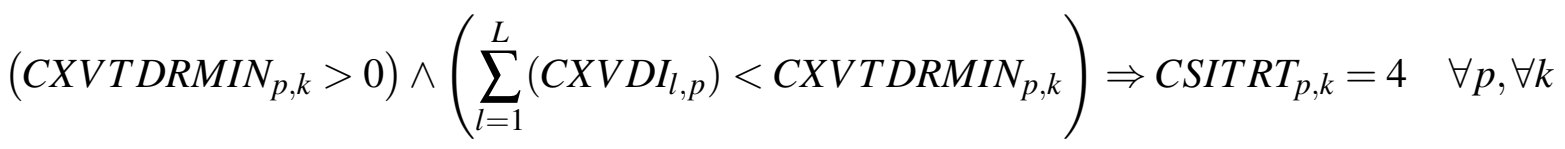

$$
\begin{array}{ll}
C X V D T D_{p, k}=0 & (\forall p, \forall k) \in\left(\operatorname{CSITRT}_{p, k}=4\right) \\
C X V D T R_{p, k}=0 & (\forall p, \forall k) \in\left(\operatorname{CSITRT}_{p, k}=4\right) \\
C X V D T T D_{p, k}=C X V D T D_{p, k}+C X V D T R_{p, k} & (\forall p, \forall k) \in\left(\operatorname{CSITRT}_{p, k}=4\right) \\
C X V R T D_{p, k}=L & (\forall p, \forall k) \in\left(\operatorname{CSITRT}_{p, k}=4\right) \\
\operatorname{CXVRTR}_{p, k}=C X V T D R M I N_{p, k}-\sum_{l=1}^{L}\left(C X V D I_{l, p}\right) & (\forall p, \forall k) \in\left(\operatorname{CSITRT}_{p, k}=4\right)
\end{array}
$$


Compondo a situação 1 para todos os produtos, gera-se a equação (6.47).

$C X V M R D_{k}=\sum_{p=1}^{P}\left(C X V R D E M I N_{p, k}\right)$

Compondo as três últimas situações para todos os produtos, então tem-se as equações (6.48) e (6.49).

$$
\begin{array}{ll}
C X V M D T_{k}=\max _{p}\left\{\max \left(C X V D T T D_{p, k}, C X V R T D_{p, k}\right)\right\} & \forall k \\
C X V M R T_{k}=\sum_{p=1}^{P}\left(C X V R T R_{p, k}\right) & \forall k
\end{array}
$$

A composição de todas as situações resulta na equação (6.50).

$$
C X M D F M I N_{k}=\max \left\{\left(C X V M D T_{k}+C X V M R T_{k}\right), C X V M R D_{k}\right\}
$$

Para as movimentações do terminal para a refinaria a proposição lógica (6.51) representa a identificação da situação 2 . As equações (6.52) representam os cálculos dos parâmetros relativos à situação 2 .

$$
\begin{array}{ll}
\left(C X V R D R M I N_{p, k}>0\right) \wedge\left(\sum_{l=1}^{L}\left(C X V D I_{l, p}\right)>0\right) \wedge & \\
\left(\sum_{l=1}^{L}\left(C X V D I_{l, p}\right)>C X V R D R M I N_{p, k}\right) \Rightarrow \operatorname{CSITT}_{p, k}=2 & \forall p, \forall k \\
C L M I N_{p}=\min _{l}\left\{l \cdot X V D I_{l, p}\right\} & \forall p \\
C X V D R D_{p, k}=\left(C L M I N_{p}-1\right) & (\forall p, \forall k) \in\left(\operatorname{CSITTR}_{p, k}=2\right) \\
C X V D R R_{p, k}=C X V R D R M I N_{p, k} & (\forall p, \forall k) \in\left(\operatorname{CSITTR}_{p, k}=2\right) \\
C X V D R T D_{p, k}=C X V D R D_{p, k}+C X V D R R_{p, k} & (\forall p, \forall k) \in\left(\operatorname{CSITTR}_{p, k}=2\right) \\
C X V T R D_{p, k}=0 & (\forall p, \forall k) \in\left(\operatorname{CSITTR}_{p, k}=2\right) \\
C X V T R R_{p, k}=0 &
\end{array}
$$

Para as movimentações do terminal para a refinaria a proposição lógica (6.53) representa a identificação da situação 3. As equações (6.54) representam os cálculos 
dos parâmetros relativos à situação 3 .

$$
\begin{aligned}
& \left(C X V \operatorname{RDRMIN}_{p, k}>0\right) \wedge\left(\sum_{l=1}^{L}\left(C X V D I_{l, p}\right)>0\right) \wedge \\
& \left(\sum_{l=1}^{L}\left(\operatorname{CXVDI}_{l, p}\right)=C X V \operatorname{RDRMIN}_{p, k}\right) \Rightarrow \operatorname{CSITTR}_{p, k}=3 \quad \forall p, \forall k
\end{aligned}
$$

$\operatorname{CLMIN}_{p}=\min _{l}\left\{l \cdot X V D I_{l, p}\right\} \quad \forall p$

$C X V \operatorname{DRD}_{p, k}=\left(\operatorname{CLMIN}_{p}-1\right) \quad(\forall p, \forall k) \in\left(\operatorname{CSITTR}_{p, k}=3\right)$

$C X V D R R_{p, k}=C X V R D R M I N N_{p, k} \quad(\forall p, \forall k) \in\left(\operatorname{CSITTR}_{p, k}=3\right)$

$C X V D R T D_{p, k}=C X V D R D_{p, k}+C X V D R R_{p, k} \quad(\forall p, \forall k) \in\left(\operatorname{CSITTR}_{p, k}=3\right)$

$C X V T R D_{p, k}=0$

$(\forall p, \forall k) \in\left(\operatorname{CSITTR}_{p, k}=3\right)$

$C X V T R R_{p, k}=0$

$(\forall p, \forall k) \in\left(C S I T T R_{p, k}=3\right)$

Para as movimentações do terminal para a refinaria a proposição lógica (6.55) representa a identificação da situação 4. As equações (6.56) representam os cálculos dos parâmetros relativos à situação 4.

$\left(C X V R D R M I N_{p, k}>0\right) \wedge\left(\sum_{l=1}^{L}\left(C X V D I_{l, p}\right)<C X V R D R M I N_{p, k}\right) \Rightarrow \operatorname{CSITTR}_{p, k}=4 \quad \forall p, \forall k$

$$
\begin{array}{ll}
C X V D R D_{p, k}=0 & (\forall p, \forall k) \in\left(\operatorname{CSITTR}_{p, k}=4\right) \\
C X V D R R_{p, k}=0 & (\forall p, \forall k) \in\left(\operatorname{CSITTR}_{p, k}=4\right) \\
C X V D R T D_{p, k}=C X V D R D_{p, k}+C X V D R R_{p, k} & (\forall p, \forall k) \in\left(\operatorname{CSITTR}_{p, k}=4\right) \\
C X V T R D_{p, k}=L & (\forall p, \forall k) \in\left(\operatorname{CSITTR}_{p, k}=4\right) \\
C X V T R R_{p, k}=C X V R D R M I N_{p, k}-\sum_{l=1}^{L}\left(C X V D I_{l, p}\right) & (\forall p, \forall k) \in\left(\operatorname{CSITTR}_{p, k}=4\right)
\end{array}
$$


A composição da situação 1 para todos os produtos resulta na equação (6.57).

$C X V M T D_{k}=\sum_{p=1}^{P}\left(C X V T D E M I N_{p, k}\right)$

As três últimas situações para todos os produtos resultam nas equações (6.58) e (6.59).

$C X V M D R_{k}=\max _{p}\left\{\max \left(C X V D R T D_{p, k}, C X V T R D_{p, k}\right)\right\}$

$C X V M T R_{k}=\sum_{p=1}^{P}\left(C X V T R R_{p, k}\right)$

A composição de todas as situações gera a equação (6.60).

$C X M D R M I N_{k}=\max \left\{\left(C X V M D R_{k}+C X V M T R_{k}\right), C X V M T D_{k}\right\}$

Então como limites das quantidades mínimas a serem bombeadas podem-se definir as equações (6.61) e (6.62). Podem-se também definir quantidades mínimas a serem bombeadas de cada produto pelas restrições (6.63) e (6.64).

$$
\begin{array}{cc}
\sum_{k^{\prime}=1}^{k}\left(X M D F_{k^{\prime}}\right) \geq C X M D F M I N_{k} & \forall k \in\left(\operatorname{CXMDFMIN}_{k}>0\right) \\
\sum_{k^{\prime}=1}^{k}\left(X M D R_{k^{\prime}}\right) \geq C X M D R M I N_{k} & \forall k \in\left(\operatorname{CXMDRMIN}_{k}>0\right) \\
\sum_{k^{\prime}=1}^{k}\left(X V R D E_{p, k^{\prime}}\right) \geq \max \left\{C X V R T R_{p, k}, C X V R D E M I N_{p, k}\right\} & (\forall p, \forall k) \in\left\{\left(C X V R T R_{p, k}>0\right) \vee\left(C X V \operatorname{RDEMIN}_{p, k}>0\right)\right\} \\
\sum_{k^{\prime}=1}^{k}\left(X V T D E_{p, k^{\prime}}\right) \geq \max \left\{C X V T R R_{p, k}, C X V T D E M I N_{p, k}\right\} & (\forall p, \forall k) \in\left\{\left(C X V T R R_{p, k}>0\right) \vee\left(C X V T D E M I N_{p, k}>0\right)\right\}
\end{array}
$$


Portanto o modelo proposto (MCCT) nesta variação é da seguinte forma:

\section{MCCT:}

Minimizar (4.40)

sujeito a:

(4.3), (4.4), (4.8) e (4.9) restrições de armazenamento da refinaria

(4.6) e (4.7) equações de troca entre refinaria e duto

(4.10), (4.11), (4.15) e (4.16) restrições de armazenamento no terminal

(4.13) e (4.14) equações de troca entre terminal e duto

(4.17), (4.31), (4.32), (4.33), (4.34) e (4.35) restrições que representam os estados do duto

(4.36), (4.37), (4.38) e (4.39) restrições de interface de produtos dentro do duto

(6.61) e (6.62) limite da quantidade mínima a ser bombeada

(6.63) e (6.64) limite da quantidade mínima de cada produto a ser bombeada

$$
\begin{aligned}
& \left(X V R D E_{p, k}, X V T D E_{p, k}\right) \in\{0 ; 1\} \\
& \left(X M D F_{k}, X M D R_{k}, X M D N_{k}\right) \in[0 ; 1] \\
& \left(X V R D R_{p, k}, X V T D R_{p, k}, X V D_{l, p, k}\right) \in[0 ; 1] \\
& \left(X I P R D_{p, p^{\prime}, k}, X I P T D_{p, p^{\prime}, k}\right) \in[0 ; 1] \\
& \left(V R_{p, k}, V R D R_{p, k}, V R D E_{p, k}, V T_{p, k}, V T D R_{p, k}, V T D E_{p, k}, V D_{l, p, k}, C U S T O\right) \geq 0
\end{aligned}
$$

\subsection{RESULTADOS}

Note que os modelos definidos neste capítulo acima não alteram o resultado final porque os cortes desenvolvidos somente eliminam parte do espaço relaxado de soluções. Portanto, a única alteração é no desempenho do tempo computacional.

Assim como no item 5.1 o sistema computacional utilizado para resolver os modelos de otimização foi o seguinte: GAMS (General Algebraic Modeling System) versão 22.0, utilizando o solver CPLEX versão 10.0.1, ambos em um microcomputador com 
4 processadores Intel Pentium Xeon 4 - $2.4 \mathrm{GHz}, 512$ Mb de memória RAM com o sistema operacional Linux.

A tabela 6.5 quantifica o tempo computacional para atingir a otimalidade global em cada um dos modelos.

Tabela 6.5: Comparação do tempo computacional

\begin{tabular}{|l|c|c|c|}
\hline \multicolumn{1}{|c|}{ Modelo } & Tempo & Iterações & Nós \\
\hline sem corte (Modelo MS) & computacional & & \\
\hline corte simples (Modelo MCS) & $18 \mathrm{~h} 21 \mathrm{~min}$ & 276.828 .904 & 8.218 .964 \\
\hline $\begin{array}{l}\text { corte simples para cada instante } k \\
\text { (modelo MCST) }\end{array}$ & $17 \mathrm{~h} 57 \mathrm{~min}$ & 235.757 .457 & 7.092 .922 \\
\hline corte completo (modelo MCC) & $08 \mathrm{~h} 20 \mathrm{~min}$ & 73.294 .782 & 1.291 .765 \\
\hline $\begin{array}{l}\text { corte completo para cada instante } k \\
\text { (modelo MCCT) }\end{array}$ & $02 \mathrm{~h} 26 \mathrm{~min}$ & 39.446 .705 & 766.428 \\
\hline
\end{tabular}

A tabela 6.6 quantifica o número de variáveis binárias e contínuas e o número de equações e inequações para cada um dos modelos.

Tabela 6.6: Estatística dos modelos

\begin{tabular}{|l|c|c|c|}
\hline \multicolumn{1}{|c|}{ Modelo } & variáveis & variáveis & equações e \\
binárias & contínuas & inequações \\
\hline sem corte (Modelo MS) & 96 & 1.073 & 1.965 \\
\hline corte simples (Modelo MCS) & 96 & 1.073 & 1.967 \\
\hline $\begin{array}{l}\text { corte simples para cada instante } k \text { (mo- } \\
\text { delo MCST) }\end{array}$ & 96 & 1.073 & 1.971 \\
\hline
\end{tabular}




\begin{tabular}{|l|c|l|c|}
\hline \multicolumn{1}{|c|}{ Modelo } & variáveis & variáveis & equações e \\
binárias & contínuas & inequações \\
\hline corte completo (modelo MCC) & 96 & 1.073 & 1.968 \\
\hline $\begin{array}{l}\text { corte completo para cada instante } k \text { (mo- } \\
\text { delo MCCT) }\end{array}$ & 96 & 1.073 & 1.974 \\
\hline
\end{tabular}

Com a adição ao modelo do corte simples (modelo MCS) para o problema houve a redução de $16,0 \%$ no tempo computacional. No caso da adição ao modelo do corte completo por tempo (modelo MCCT) houve a redução de 88,9\% no tempo computacional. Apesar da redução significativa, o tempo apresentado é ainda bastante elevado para a dimensão do problema. 


\section{REFORMULAÇÃO DO CUSTO DE ESTOCAGEM NO MODELO MILP}

\subsection{INTRODUÇÃO}

Neste capítulo é desenvolvida uma reformulação da função objetivo, pela eliminação do custo de estocagem e pela adição de uma penalização quando fora de uma faixa de estoque desejável e os resultados associados. A motivação desta reformulação é a representação mais aderente do modelo à operação.

No item 7.2 é descrito a reformulação na função objetivo. No item 7.3 é apresentado a formulação da penalização quando fora de uma faixa. No item 7.4 é apresentado os resultados da reformulação e são discutidos os resultados.

\subsection{DESCRIÇÃO}

Como discutido no item 5.2.3 os custos de estocagem influenciam diretamente o resultado da busca por uma solução, incluindo-se o custo de estocagem dentro do duto. Pelas hipóteses H4.4, H4.5 e H4.6, a soma dos volumes da refinaria, terminal e duto por produto são bem definidas ao longo do horizonte. Da forma modelada até o momento, a busca de uma melhor solução fica restrita à busca do local mais barato para estocar os produtos.

Esta situação de simples busca pelo local de menor custo de estoque pode ser evitada pela observação de como as pessoas envolvidas com os programadores de dutos racionalizam o processo. Assim é sugerida uma variação na função objetivo (eq. 4.40), onde os custos associados a estocagem são removidos, no entanto foram associadas penalidades a um estoque fora de uma faixa de operação desejada.

Esta faixa de operação desejável tem dois limites: 
- Inferior, que pode ser associado ao risco de acontecer uma falha da execução da programação; quando ocorre esta falha existe um custo associado que pode ter várias origens, como por exemplo uma multa pela não entrega do produto, ou uma redução de uma vazão de carga de uma unidade de processo, diminuindo a produtividade da mesma. Neste caso, uma eventual falha não incorre em todo o custo descrito. $O$ custo descrito neste item é associado à violação deste limite;

- Superior, de forma semelhante ao limite inferior, este limite pode ser associado ao risco de acontecer uma falha da execução da programação, entretanto também pode ser associado ao excesso de um produto estocado em um determinado local, o que é equivalente a um recurso imobilizado na forma de produto. $\mathrm{O}$ custo associado a este recurso imobilizado é equivalente à perda do rendimento de uma aplicação financeira de valor equivalente. A violação deste limite é associada ao custo descrito neste item.

Desta forma o programador atende as produções, os consumos e as demandas e incorpora os riscos associados às incertezas e situações não previstas por motivos diversos.

\subsection{MODELAGEM}

A modelagem deste custo de estocagem na função objetivo requer outras variáveis e parâmetros.

Na tabela 7.1 são apresentadas as variáveis acrescentadas ao modelo, além de sua identificação e descrição. Note que todas as variáveis nesta tabela são não negativas.

Tabela 7.1: Variáveis associadas a reformulação

\begin{tabular}{|c|l|}
\hline \multicolumn{1}{|c|}{ Variável } & \multicolumn{1}{c|}{ Descrição } \\
\hline$V R D L I_{p, k}$ & $\begin{array}{l}\text { Volume da refinaria abaixo do limite inferior da faixa desejável do } \\
\text { produto } p \text { no instante } k .\end{array}$ \\
\hline
\end{tabular}




\begin{tabular}{|l|l|}
\hline \multicolumn{1}{|c|}{ Variável } & \multicolumn{1}{c|}{ Descrição } \\
\hline$V R D L S_{p, k}$ & $\begin{array}{l}\text { Volume da refinaria acima do limite superior da faixa desejável } \\
\text { do produto } p \text { no instante } k .\end{array}$ \\
\hline$V T D L I_{p, k}$ & $\begin{array}{l}\text { Volume do terminal abaixo do limite inferior da faixa desejável do } \\
\text { produto } p \text { no instante } k .\end{array}$ \\
\hline$V T D L S_{p, k}$ & $\begin{array}{l}\text { Volume do terminal acima do limite superior da faixa desejável } \\
\text { do produto } p \text { no instante } k .\end{array}$ \\
\hline
\end{tabular}

A tabela 7.2 apresenta os parâmetros acrescentados ao modelo, com a correspondente a identificação e descrição.

Tabela 7.2: Parâmetros associados à reformulação

\begin{tabular}{|l|l|}
\hline \multicolumn{1}{|c|}{ Parâmetro } & \multicolumn{1}{c|}{ Descrição } \\
\hline$C V R M I N D_{p}$ & Volume mínimo do produto $p$ desejável da refinaria. \\
\hline$C V R M A X D_{p}$ & Volume máximo do produto $p$ desejável da refinaria. \\
\hline$C V T M I N D_{p}$ & Volume mínimo do produto $p$ desejável do terminal. \\
\hline$C V T M A X D_{p}$ & Volume máximo do produto $p$ desejável do terminal. \\
\hline$C P E R D L I_{p}$ & $\begin{array}{l}\text { Penalização (custo) do estoque da refinaria abaixo do limite infe- } \\
\text { rior do estoque desejável do produto } p \text {. }\end{array}$ \\
\hline$C P E R D L S_{p}$ & $\begin{array}{l}\text { Penalização (custo) do estoque da refinaria acima do limite su- } \\
\text { perior do estoque desejável do produto } p \text {. }\end{array}$ \\
\hline$C P E T D L I_{p}$ & $\begin{array}{l}\text { Penalização (custo) do estoque do terminal abaixo do limite infe- } \\
\text { rior do estoque desejável do produto } p \text {. }\end{array}$ \\
\hline$C P E T D L S_{p}$ & $\begin{array}{l}\text { Penalização (custo) do estoque do terminal acima do limite su- } \\
\text { perior do estoque desejável do produto } p \text {. }\end{array}$ \\
\hline
\end{tabular}


A figura 7.1 ilustra de maneira detalhada as variáveis e parâmetros do armazenamento representativo da refinaria.

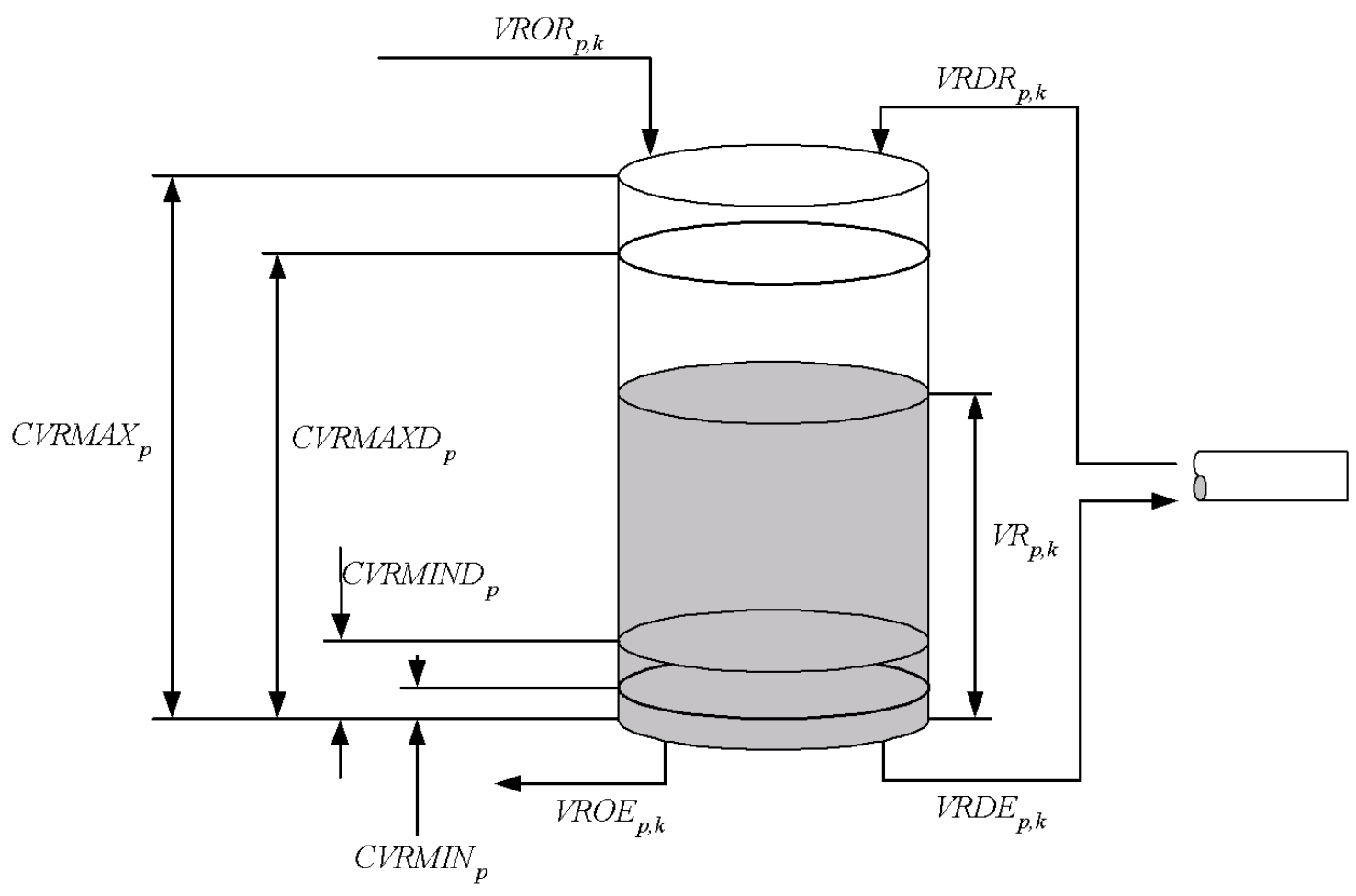

Figura 7.1: Representação do armazenamento na refinaria

A figura 7.2 ilustra de maneira detalhada as variáveis e parâmetros do armazenamento representativo do terminal.

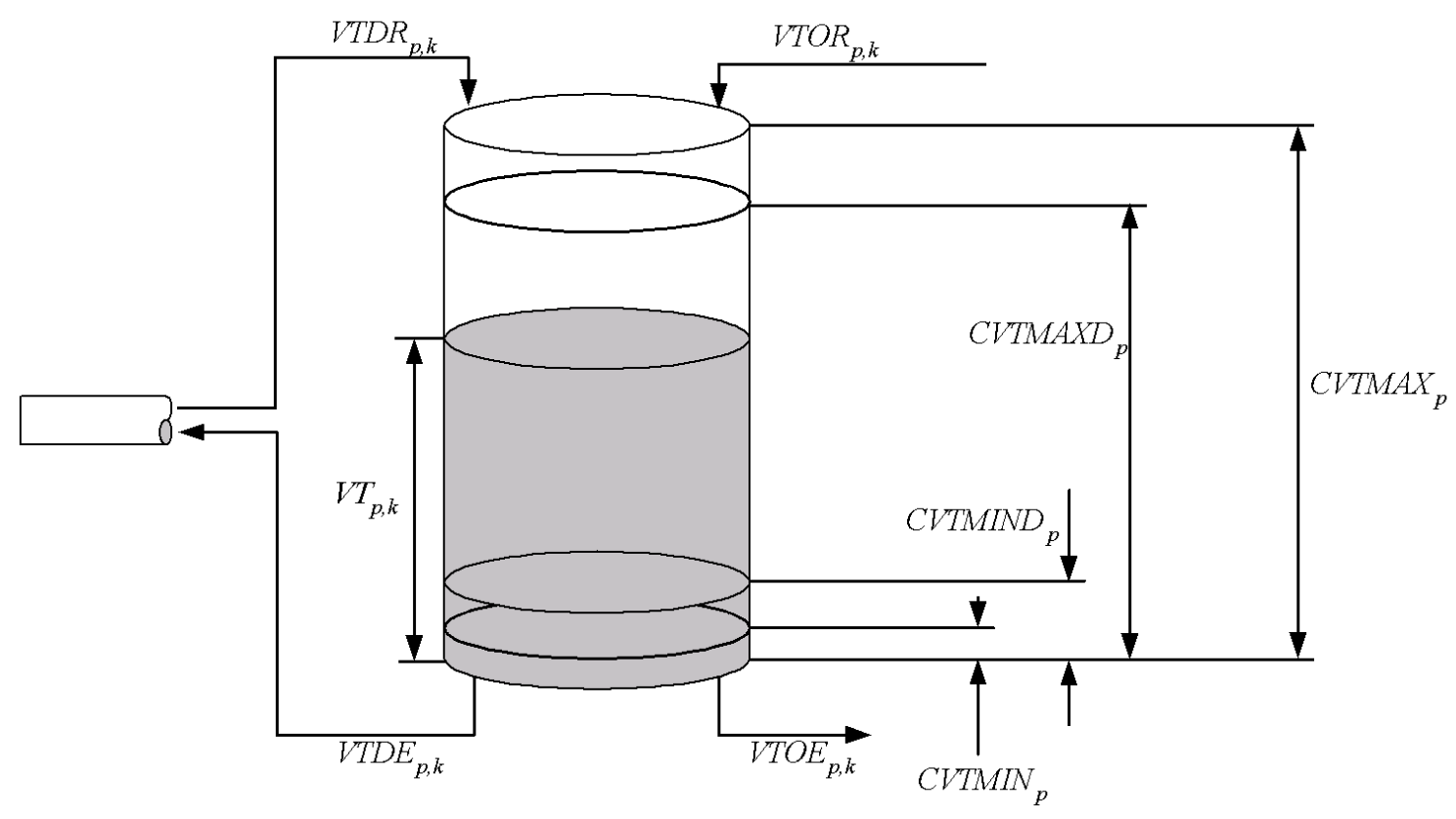

Figura 7.2: Representação do armazenamento no terminal 
A formulação para determinação dos volumes fora dos limites desejáveis é a seguinte:

$\begin{array}{ll}V R D L I_{p, k} \geq C V R M I N D_{p}-V R_{p, k} & \forall p, \forall k \\ V R D L S_{p, k} \geq V R_{p, k}-C V R M A X D_{p} & \\ & \\ V T D L I_{p, k} \geq C V T M I N D_{p}-V T_{p, k} & \forall p, \forall k \\ V T D L S_{p, k} \geq V T_{p, k}-C V T M A X D_{p} & \forall p, \forall k\end{array}$

A formulação da função objetivo com a remoção dos custos associados aos estoques da refinaria e do terminal, e com a adição das penalizações associadas à faixa de estoque desejável é dada por (7.3).

CUSTO $=\left[\sum_{p=1}^{P} \sum_{k=1}^{K}\left(\right.\right.$ CCBRD $_{p} \cdot$ VRDE $\left._{p, k}\right)+\sum_{p=1}^{P} \sum_{k=1}^{K}\left(\right.$ CCBTD $\left.\left._{p} \cdot V T D E_{p, k}\right)\right]$
$+\left[\sum_{p=1}^{P} \sum_{p^{\prime}=1}^{P^{\prime}} \sum_{k=1}^{K}\left(C C I P_{p, p^{\prime}} \cdot X I P R D_{p, p^{\prime}, k}\right)+\sum_{p=1}^{P} \sum_{p^{\prime}=1}^{P^{\prime}} \sum_{k=1}^{K}\left(C C I P_{p, p^{\prime}} \cdot X I P T D_{p, p^{\prime}, k}\right)\right]$
$+\left[\sum_{p=1}^{P} \sum_{k=1}^{K}\left(\right.\right.$ VRDLI $_{p, k} \cdot C P E R D L I_{p}+V R D L S_{p, k} \cdot C P E R D L S_{p}$

$$
\left.\left.+V T D L I_{p, k} \cdot C P E T D L I_{p}+V T D L S_{p, k} \cdot C P E T D L S_{p}\right)\right] \cdot C T
$$

Nesta função a primeira parcela representa os custos de bombeamento, a segunda parcela representa os custos de interface e terceira parcela representa as penalizações dos estoques fora dos limites desejáveis. 
Este modelo (MPE) contempla a variação descrita no item 6.3.1 e portanto tem a seguinte forma:

\section{MPE:}

Minimizar (7.3)

sujeito a:

(4.3), (4.4), (4.8) e (4.9) restrições de armazenamento da refinaria

(4.6) e (4.7) equações de troca entre refinaria e duto

(4.10), (4.11), (4.15) e (4.16) restrições de armazenamento no terminal

(4.13) e (4.14) equações de troca entre terminal e duto

(4.17), (4.31), (4.32), (4.33), (4.34) e (4.35) restrições que representam os estados do duto

(4.36), (4.37), (4.38) e (4.39) restrições de interface de produtos dentro do duto

(6.61) e (6.62) limite da quantidade mínima a ser bombeada

(6.63) e (6.64) limite da quantidade mínima de cada produto a ser bombeada

(7.1) e (7.2) inequações que representam os volumes fora dos limites

desejáveis

$$
\begin{aligned}
& \left(X V R D E_{p, k}, X V T D E_{p, k}\right) \in\{0 ; 1\} \\
& \left(X M D F_{k}, X M D R_{k}, X M D N_{k}\right) \in[0 ; 1] \\
& \left(X V R D R_{p, k}, X V T D R_{p, k}, X V D_{l, p, k}\right) \in[0 ; 1] \\
& \left(X I P R D_{p, p^{\prime}, k}, X I P T D_{p, p^{\prime}, k}\right) \in[0 ; 1] \\
& \left(V R_{p, k}, V R D R_{p, k}, V R D E_{p, k}, V T_{p, k}, V T D R_{p, k}, V T D E_{p, k}, V D_{l, p, k}, C U S T O\right) \geq 0 \\
& \left(V R D L I_{p, k}, V R D L S_{p, k}, V T D L I_{p, k}, V T D L S_{p, k}\right) \geq 0
\end{aligned}
$$

Note que o modelo MPE contém todos os cortes desenvolvidos no capítulo 6 , dados pelas inequações (6.61) a (6.64).

Neste tipo de formulação utilizando a penalização como parte da função objetivo há dificuldades associadas que são como determinar os valores das mesmas. Como 
a princípio estes valores são subjetivos, então deve-se primeiro determinar um valor econômico adequado. O mesmo vale para determinação dos limites das faixas.

\subsection{RESULTADOS COMPUTACIONAIS DOS MODELOS}

Utiliza-se o mesmo sistema protótipo descrito no item 5.2.1 como base e as variações descritas acima.

Os parâmetros de volumes relacionados ao estoque fora da faixa desejável estão descritos na tabela 7.3.

Tabela 7.3: Parâmetros do protótipo p/ faixa da tancagem

\begin{tabular}{|c|c|c|c|c|}
\hline produto $(p)$ & $\begin{array}{c}C \text { VRMIND }_{p} \\
\left(\mathrm{~m}^{3}\right)\end{array}$ & $\begin{array}{c}C V R M A X D_{p} \\
\left(\mathrm{~m}^{3}\right)\end{array}$ & $\begin{array}{c}C V T \text { MIND } \\
\left(\mathrm{m}^{3}\right)\end{array}$ & $\begin{array}{c}C V T M A X D_{p} \\
\left(\mathrm{~m}^{3}\right)\end{array}$ \\
\hline 1 & 1.000 & 4.500 & 1.000 & 4.500 \\
\hline 2 & 1.000 & 4.500 & 1.000 & 4.500 \\
\hline 3 & 1.000 & 4.500 & 1.000 & 4.500 \\
\hline
\end{tabular}

Os parâmetros de penalização do protótipo são dados na tabela 7.4.

Tabela 7.4: Parâmetros do protótipo $\mathrm{p} /$ penalização fora da faixa

\begin{tabular}{|c|c|c|c|c|}
\hline produto $(p)$ & $\begin{array}{c}C P E R D L I_{p} \\
\left(\mathrm{R} \$ /\left(m^{3} \cdot h\right)\right)\end{array}$ & $\begin{array}{c}C P E R D L S_{p} \\
\left(\mathrm{R} \$ /\left(m^{3} \cdot h\right)\right)\end{array}$ & $\begin{array}{c}\text { CPETDLI }_{p} \\
\left(\mathrm{R} \$ /\left(m^{3} \cdot h\right)\right)\end{array}$ & $\begin{array}{c}C P E T D L S_{p} \\
\left(\mathrm{R} \$ /\left(m^{3} \cdot h\right)\right)\end{array}$ \\
\hline 1 & 50,0 & 50,0 & 60,0 & 60,0 \\
\hline 2 & 60,0 & 60,0 & 50,0 & 50,0 \\
\hline 3 & 55,0 & 55,0 & 55,0 & 55,0 \\
\hline
\end{tabular}

Os resultados otimizados são gerados e comparados com a mesma solução "manual" apresentada no item 5.2.2.

Assim como no item 5.1 o sistema computacional utilizado para resolver os modelos de otimização foi o seguinte: GAMS (General Algebraic Modeling System) versão 22.0, utilizando o solver CPLEX versão 10.0.1, ambos em um microcomputador com 4 processadores Intel Pentium Xeon 4 - $2.4 \mathrm{GHz}, 512$ Mb de memória RAM com o sistema operacional Linux.

A tabela 7.5 mostra o tempo computacional para atingir a otimalidade global em cada um dos modelos. 
Tabela 7.5: Comparação do tempo computacional para reformulação da função objetivo

\begin{tabular}{|l|c|c|c|}
\hline \multicolumn{1}{|c|}{ Modelo } & Tempo & Iterações & Nós \\
\hline computacional & & \\
\hline $\begin{array}{l}\text { corte simples (Modelo MCS + reformula- } \\
\text { ção) }\end{array}$ & 09 min56s & 2.208 .982 & 48.213 \\
\hline $\begin{array}{l}\text { corte simples para cada instante } k \text { (modelo } \\
\text { MCST + reformulação) }\end{array}$ & 05 min39s & 1.253 .741 & 27.632 \\
\hline $\begin{array}{l}\text { corte completo (modelo MCC + reformula- } \\
\text { ção) }\end{array}$ & 04 min47s & 952.605 & 15.377 \\
\hline $\begin{array}{l}\text { corte completo para cada instante } k \text { (mo- } \\
\text { delo MCCT + reformulação = MPE) }\end{array}$ & 03 min52s & 733.460 & 98.046 \\
\hline
\end{tabular}

Comparando a tabela 7.5 com a tabela 6.5 nota-se que houve, em média, uma redução de $90 \%$ no número de iterações, que foi acompanhada pela redução do tempo computacional na mesma proporção.

A tabela 7.6 apresenta a comparação dos valores das funções objetivo manual e otimizada. A tabela 7.7 apresenta a comparação entre os bombeamentos da refinaria para o terminal, e a tabela 7.8 apresenta a comparação entre os bombeamentos do terminal para a refinaria. Note que as tabelas 7.7 e 7.8 indicam os tipos de produto bombeado em cada intervalo de tempo.

Tabela 7.6: Valores da função objetivo

\begin{tabular}{|c|c|}
\hline Caso & Valor Função Objetivo \\
\hline "manual" & 214.350 \\
\hline Modelo & 190.350 \\
\hline
\end{tabular}


Tabela 7.7: Movimentações da refinaria para terminal

\begin{tabular}{|c|c|c|c|c|c|c|c|c|c|c|c|c|c|c|c|c|}
\hline Caso & \multicolumn{11}{|c|}{ tempo $(k)$} \\
& 1 & 2 & 3 & 4 & 5 & 6 & 7 & 8 & 9 & 10 & 11 & 12 & 13 & 14 & 15 & 16 \\
\hline "manual" & 3 & 3 & 3 & 3 & 3 & 3 & & & & & & & & & & \\
\hline Modelo & 3 & 3 & 3 & 3 & & & & & & & & & & & & \\
\hline
\end{tabular}

Tabela 7.8: Movimentações do terminal para refinaria

\begin{tabular}{|c|c|c|c|c|c|c|c|c|c|c|c|c|c|c|c|c|}
\hline Caso & \multicolumn{110}{|c|}{ tempo $(k)$} \\
\hline & 1 & 2 & 3 & 4 & 5 & 6 & 7 & 8 & 9 & 10 & 11 & 12 & 13 & 14 & 15 & 16 \\
\hline "manual" & & & & & & & 2 & 2 & 2 & 2 & 2 & 2 & 2 & 2 & 3 & 3 \\
\hline Modelo & & & & & 2 & 2 & 2 & 2 & 2 & 2 & 3 & 3 & 3 & 3 & 3 & 3 \\
\hline
\end{tabular}

Os perfis do duto ao longo do horizonte do tempo com o conteúdo do mesmo estão apresentados na tabela 7.9:

Tabela 7.9: Perfis do duto no caso manual e MPE

\begin{tabular}{|c|c|c|c|c|c|c|c|c|c|c|c|c|c|c|c|c|}
\hline \multirow{2}{*}{ tempo $(k)$} & \multicolumn{8}{|c|}{ Manual } & \multicolumn{8}{|c|}{ Modelo } \\
\hline & & 1 & 2 & 3 & 4 & 5 & 6 & & & 1 & 2 & 3 & 4 & 5 & 6 & \\
\hline 1 & $\rightarrow$ & 3 & 1 & 1 & 1 & 1 & 1 & & $\rightarrow$ & 3 & 1 & 1 & 1 & 1 & 1 & \\
\hline 2 & $\rightarrow$ & 3 & 3 & 1 & 1 & 1 & 1 & & $\rightarrow$ & 3 & 3 & 1 & 1 & 1 & 1 & \\
\hline 3 & $\rightarrow$ & 3 & 3 & 3 & 1 & 1 & 1 & & $\rightarrow$ & 3 & 3 & 3 & 1 & 1 & 1 & \\
\hline 4 & $\rightarrow$ & 3 & 3 & 3 & 3 & 1 & 1 & & $\rightarrow$ & 3 & 3 & 3 & 3 & 1 & 1 & \\
\hline 5 & $\rightarrow$ & 3 & 3 & 3 & 3 & 3 & 1 & & & 3 & 3 & 3 & 1 & 1 & 2 & $\leftarrow$ \\
\hline 6 & $\rightarrow$ & 3 & 3 & 3 & 3 & 3 & 3 & & & 3 & 3 & 1 & 1 & 2 & 2 & $\leftarrow$ \\
\hline 7 & & 3 & 3 & 3 & 3 & 3 & 2 & $\leftarrow$ & & 3 & 1 & 1 & 2 & 2 & 2 & $\leftarrow$ \\
\hline 8 & & 3 & 3 & 3 & 3 & 2 & 2 & $\longleftarrow$ & & 1 & 1 & 2 & 2 & 2 & 2 & $\leftarrow$ \\
\hline 9 & & 3 & 3 & 3 & 2 & 2 & 2 & $\longleftarrow$ & & 1 & 2 & 2 & 2 & 2 & 2 & $\leftarrow$ \\
\hline 10 & & 3 & 3 & 2 & 2 & 2 & 2 & $\leftarrow$ & & 2 & 2 & 2 & 2 & 2 & 2 & $\leftarrow$ \\
\hline 11 & & 3 & 2 & 2 & 2 & 2 & 2 & $\leftarrow$ & & 2 & 2 & 2 & 2 & 2 & 3 & $\leftarrow$ \\
\hline 12 & & 2 & 2 & 2 & 2 & 2 & 2 & $\leftarrow$ & & 2 & 2 & 2 & 2 & 3 & 3 & $\leftarrow$ \\
\hline 13 & & 2 & 2 & 2 & 2 & 2 & 2 & 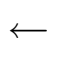 & & 2 & 2 & 2 & 3 & 3 & 3 & $\leftarrow$ \\
\hline 14 & & 2 & 2 & 2 & 2 & 2 & 2 & $\leftarrow$ & & 2 & 2 & 3 & 3 & 3 & 3 & $\leftarrow$ \\
\hline 15 & & 2 & 2 & 2 & 2 & 2 & 3 & $\leftarrow$ & & 2 & 3 & 3 & 3 & 3 & 3 & $\leftarrow$ \\
\hline
\end{tabular}




\begin{tabular}{|c|c|c|c|c|c|c|c|c|c|c|c|c|c|c|}
\hline \multirow{2}{*}{ tempo $(k)$} & \multicolumn{7}{|c|}{ Manual } & \multicolumn{7}{|c|}{ Modelo } \\
\hline & 1 & 2 & 3 & 4 & 5 & 6 & & 1 & 2 & 3 & 4 & 5 & 6 & \\
\hline 16 & 2 & 2 & 2 & 2 & 3 & 3 & $\leftarrow$ & 3 & 3 & 3 & 3 & 3 & 3 & $\leftarrow$ \\
\hline
\end{tabular}

As figuras referentes aos estoques da refinaria (7.3) e do terminal (7.4) para o caso manual e as figuras referentes aos estoques da refinaria (7.5) e do terminal (7.6) para o caso modelo, são apresentadas na seqüência:

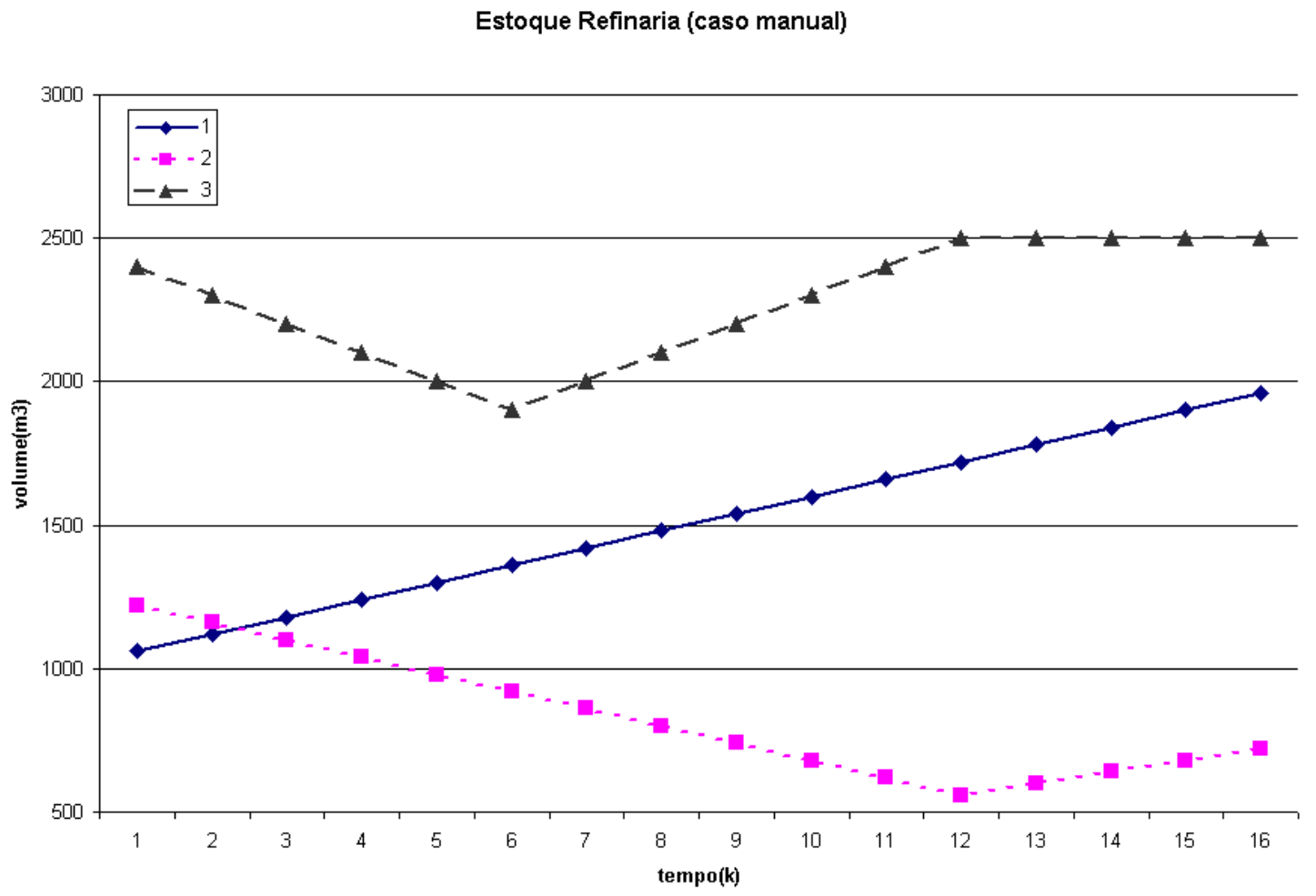

Figura 7.3: Estoque da refinaria para o caso manual 
Estoque Terminal (caso manual)

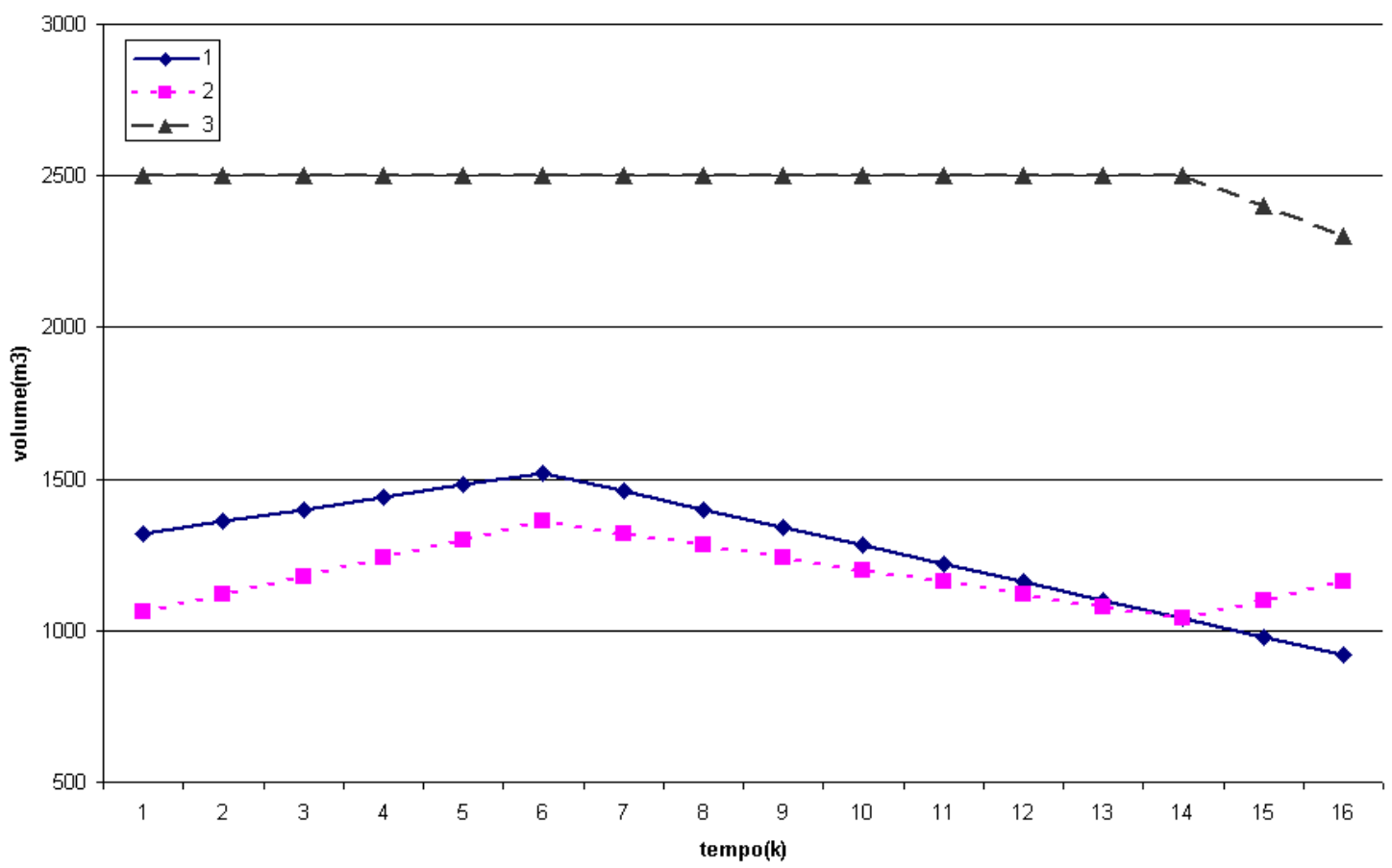

Figura 7.4: Estoque do terminal para o caso manual

Estoque Refinaria (caso modelo)

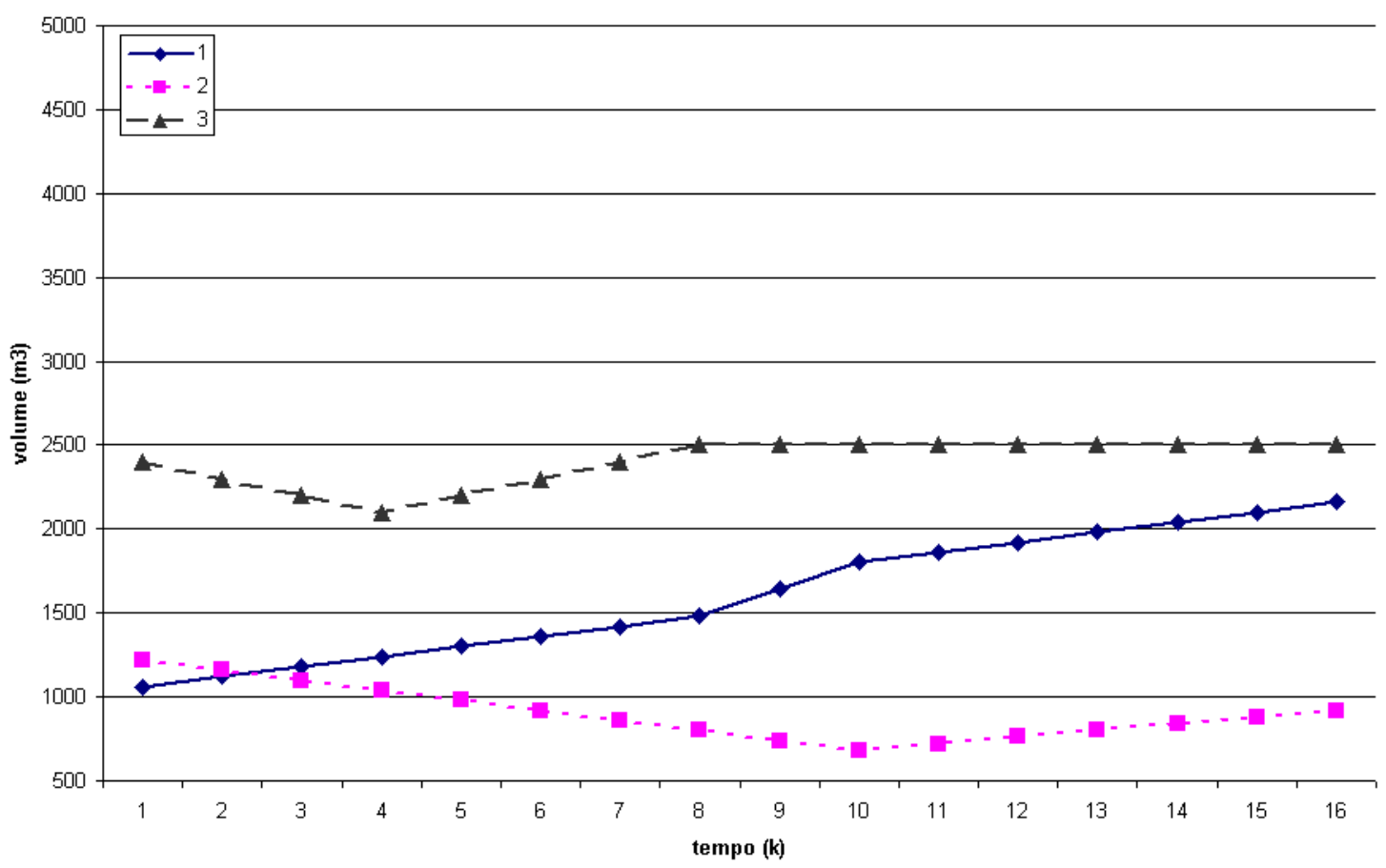

Figura 7.5: Estoque da refinaria para o caso modelo 


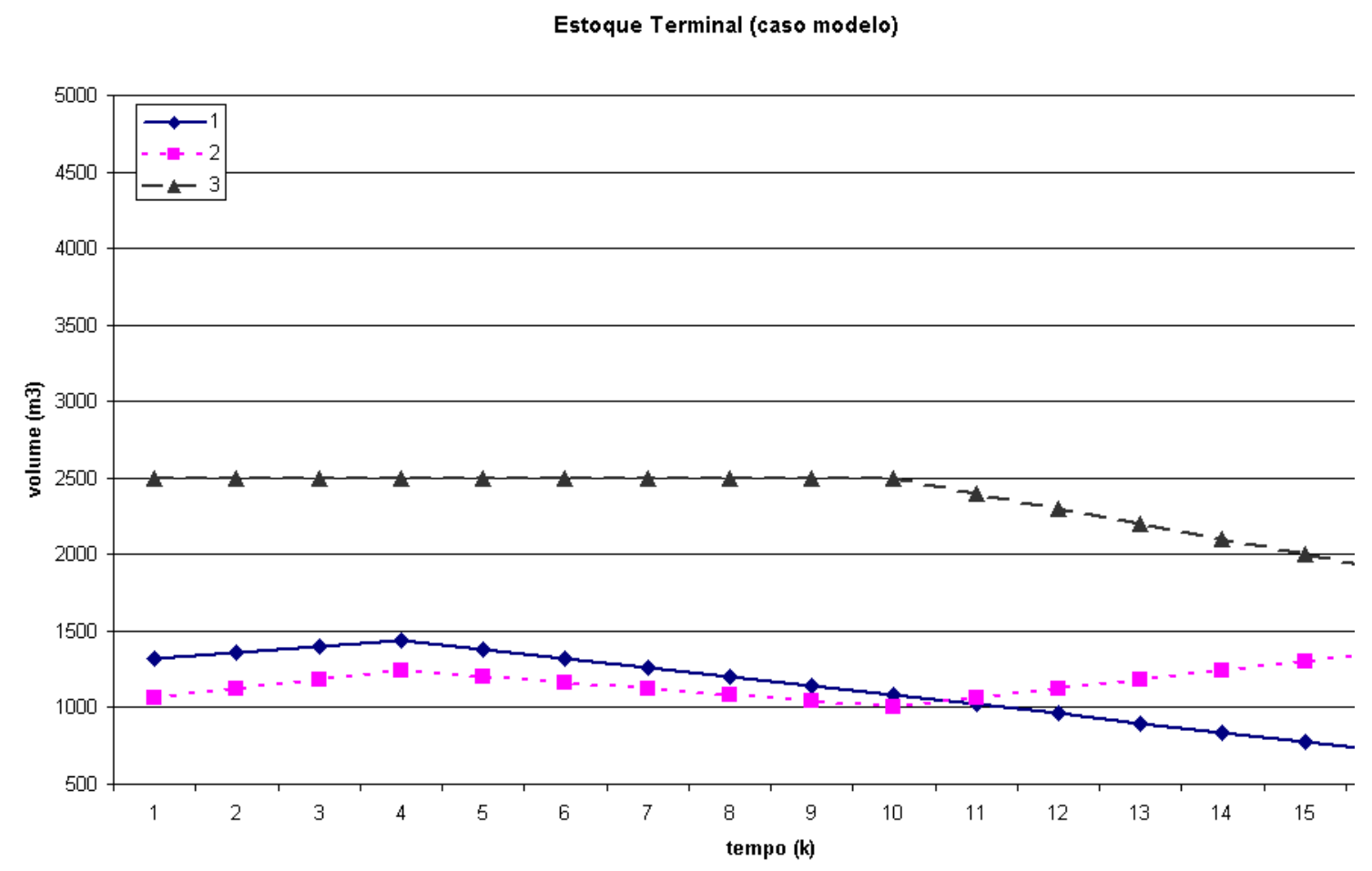

Figura 7.6: Estoque do terminal para o caso modelo

O primeiro ponto bastante interessante é o tempo computacional necessário para resolver o modelo com o caso protótipo. A provável causa da redução do tempo computacional neste caso, diferente do descrito no capítulo 5, foi a não ocorrência da degeneração do modelo (presença de múltiplas soluções com o mesmo valor de função objetivo).

Verifica-se que o sistema enviou o produto 3 da refinaria para o terminal nos 4 primeiros intervalos de tempo, na seqüência inverte o sentido do duto enviando o produto 2 do quinto ao décimo intervalo e nos intervalos subseqüentes até o último intervalo o sistema envia o produto 3.

Na solução ótima encontrada, analisando os gráficos de estoque, verifica-se que há uma tendência de manter o estoque do produto 2 na refinaria dentro da faixa desejável quando possível. O mesmo ocorre com o estoque do produto 1 no terminal. Observa-se também que o produto 3 mantém-se dentro da faixa desejável durante todo o período na refinaria e no terminal. Por estes motivos, nos primeiros 4 intervalos o sistema envia o produto 3 da refinaria para o terminal (mantendo-se dentro da faixa desejável) e neste mesmos 4 primeiros intervalos o terminal recebe o produto 1 , que se mantém o máximo possível dentro da faixa desejável. Do intervalo 5 ao 10 o 
sistema envia o produto 2 do terminal para a refinaria, para manter o máximo possível do estoque do mesmo na refinaria dentro da faixa desejável. A troca do envio do terminal para refinaria pelo produto 3 , que ocorreu no intervalo 11 ao 16, teve três motivações: o estoque do produto 2 no terminal não ficar fora do limite desejável, os produtos enviados do terminal para a refinaria a partir do intervalo 11 não atingem a refinaria, de acordo com o perfil na tabela 7.9 , e finalmente o produto 3 não fica fora da faixa desejável.

\subsection{ANÁLISE DE SENSIBILIDADE DO PARÂMETRO DE PENALIZAÇÃO}

Conforme descrito no item 7.3 o parâmetro de penalização do estoque fora do limite precisa ter um valor econômico adequado.

Em uma breve análise dos parâmetros de penalização $\left(C P E R D L I_{p}, C P E R D L S_{p}\right.$, $C P E T D L I_{p}$ e $\left.C P E T D L S_{p}\right)$ multiplicados pelo intervalo de tempo $(C T)$ em relação ao custo de bombeamento $\left(C C B R D_{p}\right.$ e $\left.C C B T D_{p}\right)$ verificou-se que para o caso protótipo ocorrem três situações distintas, que são as seguintes:

- Situação 1. O produto entre o parâmetro de penalização e o intervalo de tempo muito menor que o custo de bombeamento;

- Situação 2. O produto entre o parâmetro de penalização e o intervalo de tempo muito maior que o custo de bombeamento;

- Situação 3. O produto entre o parâmetro de penalização e o intervalo de tempo com a mesma ordem de grandeza que o custo de bombeamento.

Simplificando a explicação, a comparação feita foi entre o custo do produto fora da faixa desejável e o custo de se bombear o produto para ficar dentro da faixa.

$\mathrm{Na}$ situação $1\left(C P E R D L I_{p} \cdot C T \ll C C B T D_{p}\right.$ ou $C P E T D L I_{p} \cdot C T \ll C C B R D_{p}$ ou $C P E R D L S_{p} \cdot C T \ll C C B R D_{p}$ ou $\left.C P E T D L S_{p} \cdot C T \ll C C B T D_{p}\right)$ observou-se que na solução otimizada fornecida pelo modelo os bombeamentos entre a refinaria e o terminal e entre o terminal e a refinaria foram minimizados, isto é, somente o suficiente para atingir o limite mínimo dos estoques.

$\mathrm{Na}$ situação $2\left(C P E R D L I_{p} \cdot C T \gg C C B T D_{p}\right.$ ou $C P E T D L I_{p} \cdot C T \gg C C B R D_{p}$ ou $C P E R D L S_{p} \cdot C T \gg C C B R D_{p}$ ou $C P E T D L S_{p} \cdot C T \gg C C B T D_{p}$ ) observou-se na solução 
otimizada fornecida pelo modelo os bombeamentos realizados foram os necessários para ficar dentro do limite desejável dos estoques.

$\mathrm{Na}$ situação $3\left(C P E R D L I_{p} \cdot C T \simeq C C B T D_{p}\right.$ ou $C P E T D L I_{p} \cdot C T \simeq C C B R D_{p}$ ou $C P E R D L S_{p} \cdot C T \simeq C C B R D_{p}$ ou $C P E T D L S_{p} \cdot C T \simeq C C B T D_{p}$ ) observou-se na solução otimizada fornecida pelo modelo a situação intermediária entre 1 e 2.

As tabelas 7.10 e 7.11 apresentam a comparação das movimentações no duto de acordo com as situações descritas acima.

Tabela 7.10: Movimentações da refinaria para terminal

\begin{tabular}{|c|c|c|c|c|c|c|c|c|c|c|c|c|c|c|c|c|}
\hline \multirow{2}{*}{ Situação } & \multicolumn{16}{|c|}{ tempo $(k)$} \\
\hline & 1 & 2 & 3 & 4 & 5 & 6 & 7 & 8 & 9 & 10 & 11 & 12 & 13 & 14 & 15 & 16 \\
\hline 1 & & 1 & 1 & & & & & & & & & & & & & \\
\hline 2 & 3 & 3 & 3 & 3 & & & & & & & & & & & & \\
\hline 3 & 3 & 3 & & & & & & & & & & & & & & \\
\hline
\end{tabular}

Tabela 7.11: Movimentações do terminal para refinaria

\begin{tabular}{|c|c|c|c|c|c|c|c|c|c|c|c|c|c|c|c|c|}
\hline \multirow{2}{*}{ Situação } & \multicolumn{110}{|c|}{ tempo $(k)$} \\
\hline 1 & 1 & 2 & 3 & 4 & 5 & 6 & 7 & 8 & 9 & 10 & 11 & 12 & 13 & 14 & 15 & 16 \\
\hline 2 & & & & & 2 & 2 & 2 & 2 & 2 & 2 & 2 & 2 & & & & \\
\hline 3 & & & & & 2 & 2 & 2 & 2 & 2 & 2 & 3 & 3 & 3 & 3 & 3 & 3 \\
\hline
\end{tabular}

A figura 7.7 apresenta aos estoques da refinaria do produto $p=2$ e a figura 7.8 do terminal do produto $p=1$ para as situações acima. 


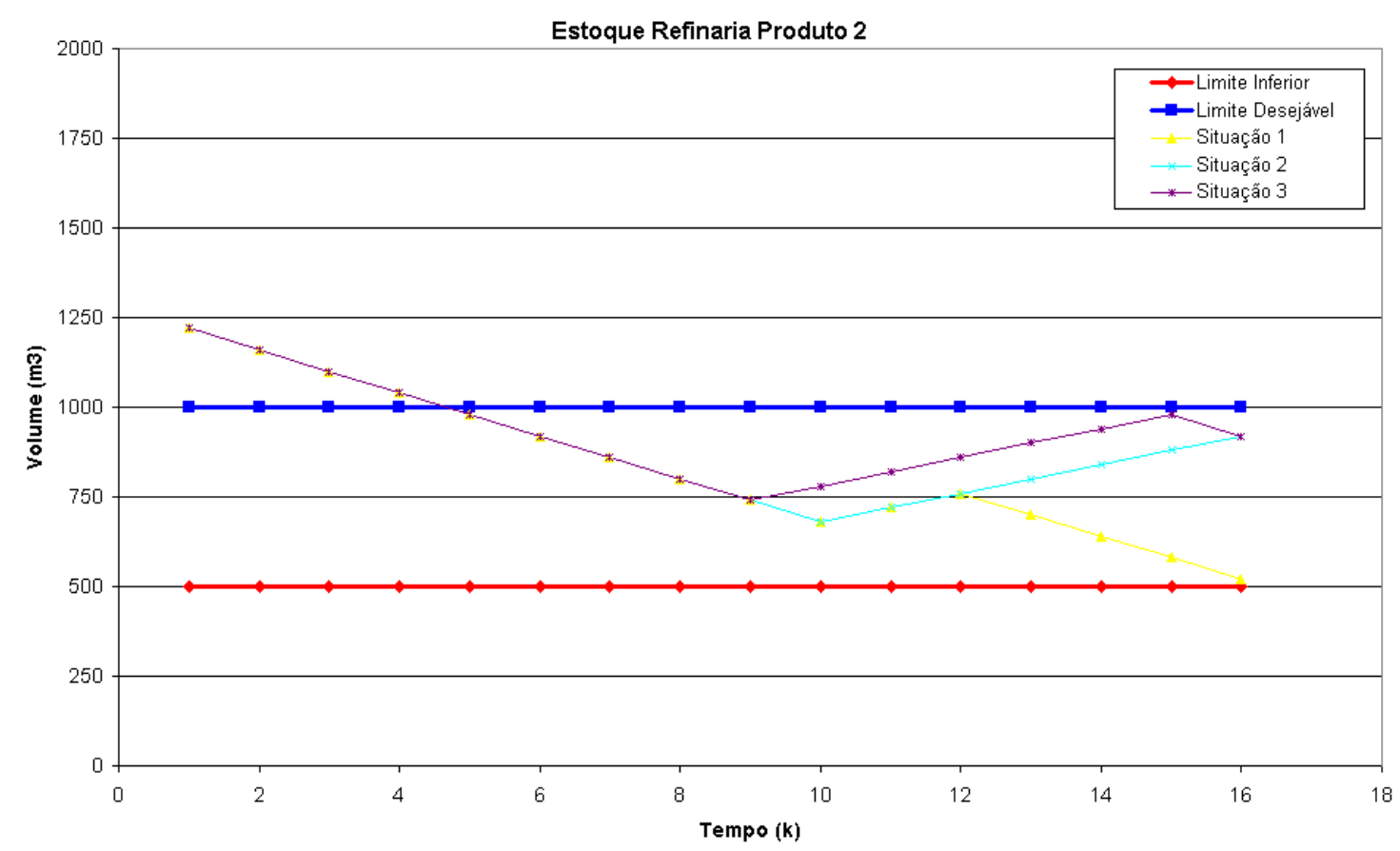

Figura 7.7: Estoque da refinaria do produto $p=2$ para as 3 situações analisadas

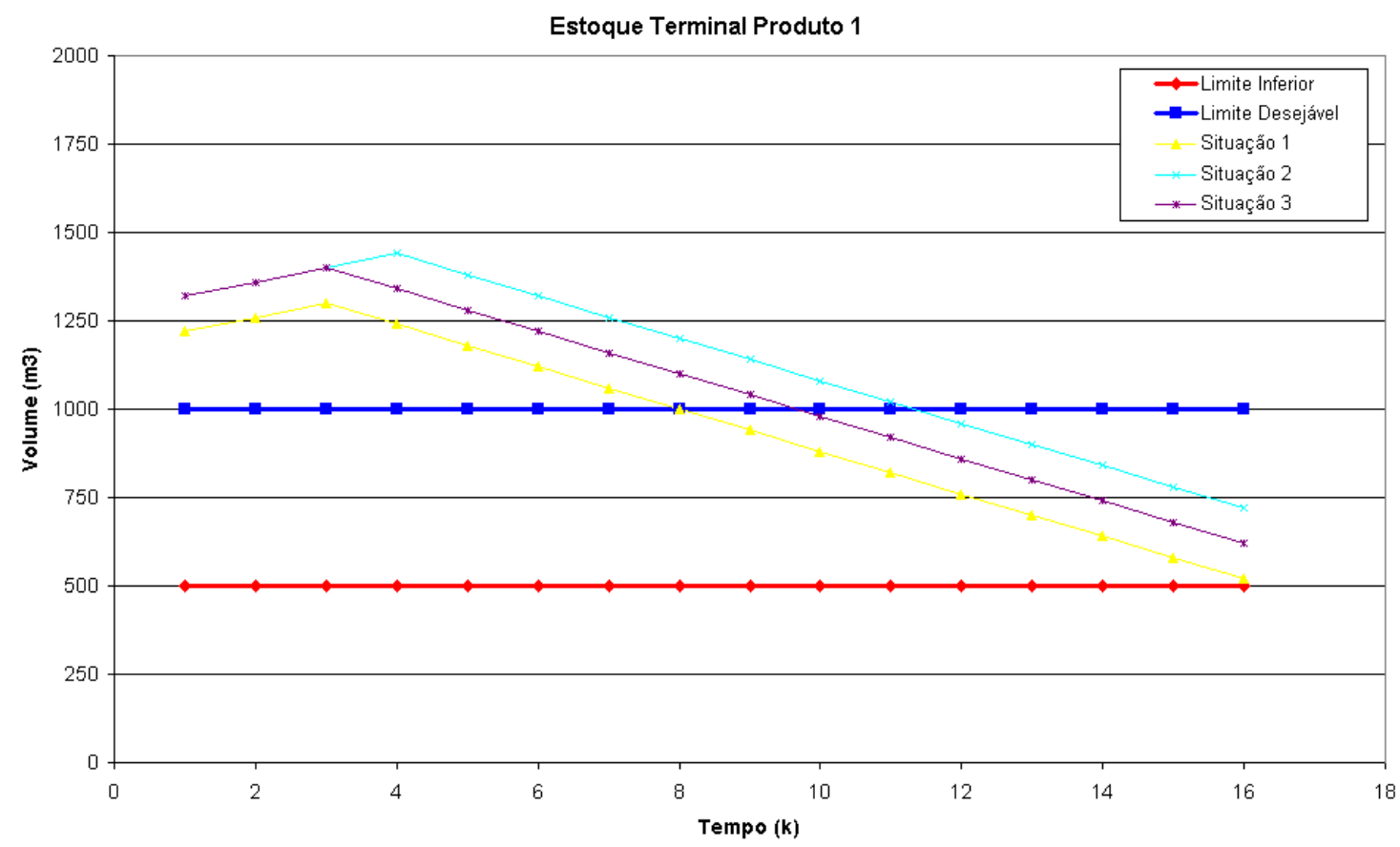

Figura 7.8: Estoque do terminal do produto $p=2$ para as 3 situações analisadas 


\section{PROGRAMAÇÃO DE DOIS TERMINAIS INTERLIGADOS}

\subsection{INTRODUÇÃO}

Este capítulo descreve um caso real do problema descrito no capítulo 3. A reversão do sentido do duto ocorre em alguns dos dutos do sistemas da rede dutoviária descrita no item 3.2.

No item 8.2 é descrito o subsistema onde ocorre a reversão de sentido do duto. No item 8.3 são apresentados os dados utilizados em um dos casos e no item 8.4 são apresentados os resultados obtidos. No item 8.5 são discutidos os resultados gerados.

\subsection{DESCRIÇÃO DO CASO REAL}

Na rede dutoviária apresentada no item 3.2 é verificada a necessidade de reversão do sentido do duto para o seguinte sistema: o terminal de São Sebastião está junto a um porto, e através deste porto recebe produtos acabados e envia produtos acabados para outras regiões ou países. O terminal de São Sebastião é interligado com o terminal de Guararema através do duto OSPLAN que transporta produtos do tipo denominado como claro. O terminal de Guararema é também interligado a outros terminais e refinarias, servindo como um entreposto dos mesmos; este terminal recebe os produtos acabados produzidos nas refinarias e estes produtos são enviados para o terminal de São Sebastião; além disto recebe os produtos acabados do terminal de São Sebastião e estes produtos são enviados para os outros terminais. A figura 8.1 ilustra a representação do sistema. As interligações descritas podem ser visualizadas na figura 3.1 do item 3.2. 


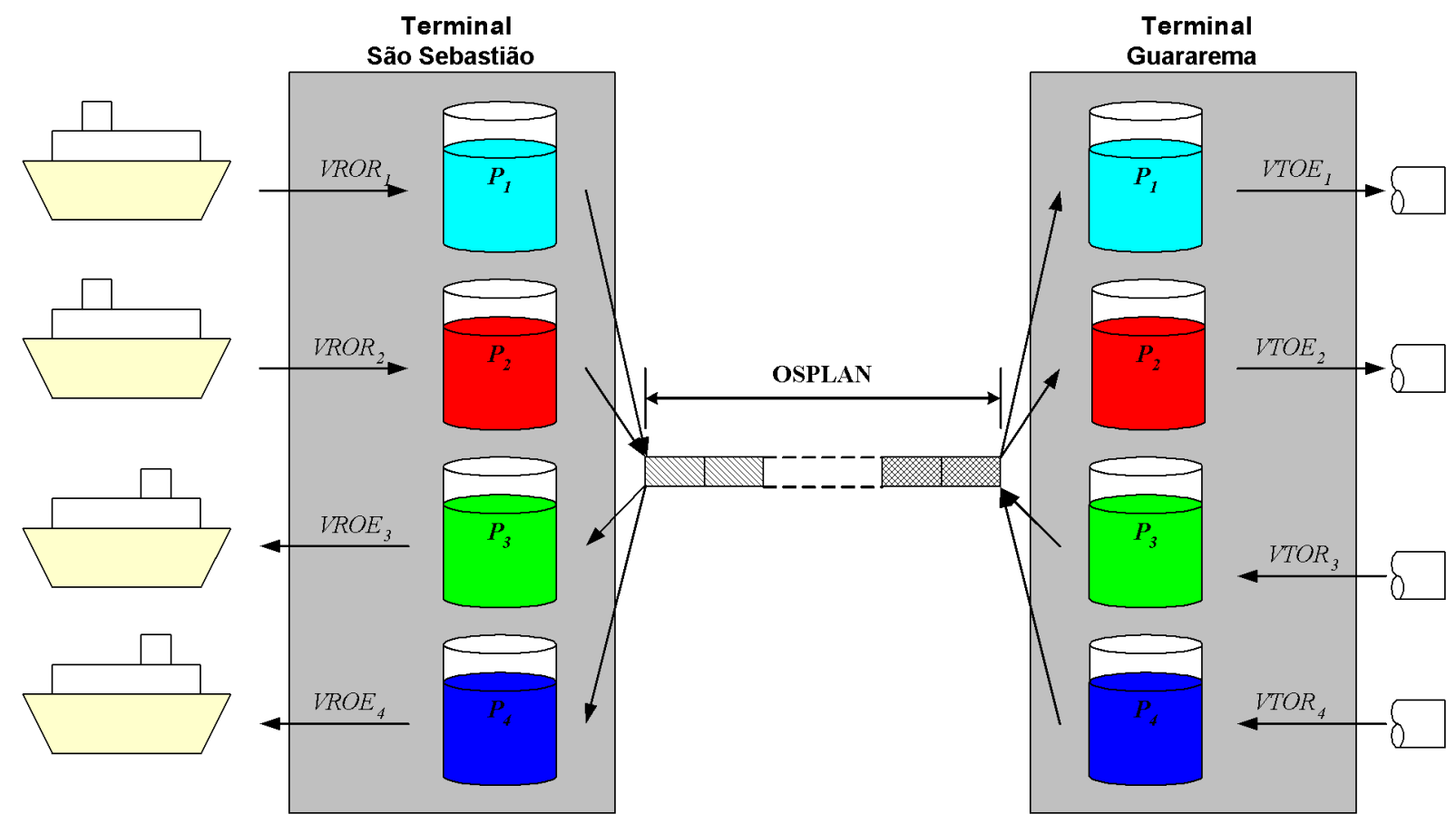

Figura 8.1: Representação do esquema do caso real de reversão do duto

No caso descrito neste item dois tipos de produtos são produzidos pelas refinarias ( $p_{3}$ e $p_{4}$ ) e enviados para o terminal de São Sebastião, e dois outros produtos são recebidos no porto do terminal de São Sebastião e enviados para outros terminais via terminal de Guararema ( $p_{1}$ e $\left.p_{2}\right)$.

Para o estudo deste caso real o terminal de São Sebastião foi adotado como a refinaria e o terminal de Guararema como se fosse o terminal. Obviamente, o duto OSPLAN é o duto descrito no modelo.

\subsection{DADOS UTILIZADOS}

Os dados utilizados para este caso real foram de operações realizadas ao longo do período selecionado.

Os dados do sistema são compostos por:

- 4 produtos distintos $(P=4)$;

- $O$ duto possui volume total de $21.000 \mathrm{~m}^{3}\left(C V D T=21.500 \mathrm{~m}^{3}\right.$ e é discretizado em 8 lotes de $2.687,5 \mathrm{~m}^{3}(L=8$ e $C V D L=2687,5)$;

- vazão volumétrica média adotada para movimentação do duto de $806 \mathrm{~m}^{3} / h$; 
- cada intervalo de tempo de aproximadamente de 3h e 20 min;

- o horizonte de tempo contém 50 intervalos que corresponde a 7 dias.

Os conjuntos que foram utilizados para este caso real são descritos na tabela 8.1. Tabela 8.1: Índices do caso real

\begin{tabular}{|l|l|}
\hline$p$ & $1 \ldots 4 \rightarrow P=4$ \\
\hline$l$ & $1 \ldots 8 \rightarrow L=8$ \\
\hline$k$ & $1 \ldots 50 \rightarrow K=50$ \\
\hline
\end{tabular}

Os parâmetros em relação à tancagem de São Sebastião (refinaria) que foram utilizados são descritos na tabela 8.2 (condição inicial e limites) e na tabela 8.3 (recebimentos e envios pré estabelecidos).

Tabela 8.2: Parâmetros do caso real para tancagem da refinaria

\begin{tabular}{|c|c|c|c|}
\hline produto $(p)$ & $C V R I_{p}\left(m^{3}\right)$ & $C V R M I N_{p}\left(m^{3}\right)$ & $C V R M A X_{p}\left(m^{3}\right)$ \\
\hline 1 & 22.068 & 0 & 26.245 \\
\hline 2 & 0 & 0 & 36.802 \\
\hline 3 & 33.753 & 0 & 52.519 \\
\hline 4 & 43.406 & 0 & 257.152 \\
\hline
\end{tabular}

Tabela 8.3: Parâmetros do caso real para tancagem da refinaria ao longo do tempo

\begin{tabular}{|c|c|c|c|}
\hline produto $(p)$ & instante $(k)$ & $C \operatorname{VROR}_{p, k}\left(m^{3}\right)$ & $C V R O E_{p, k}\left(m^{3}\right)$ \\
\hline 1 & $1 \ldots K$ & 0 & 0 \\
\hline 2 & 2 & 1.024 & 0 \\
\hline 2 & $3 \ldots 8$ & 5.502 & 0 \\
\hline 2 & 9 & 550 & 0 \\
\hline 3 & 35 & 0 & 1.490 \\
\hline 3 & 36 & 0 & 3.973 \\
\hline 3 & 37 & 0 & 3.641 \\
\hline 3 & 38 & 0 & 3.274 \\
\hline 3 & 39 & 0 & 573 \\
\hline 4 & 28 & 0 & 851 \\
\hline 4 & 29 & 0 & 2.090 \\
\hline
\end{tabular}


Os parâmetros em relação à tancagem do terminal que foram utilizados são mostrados nas tabelas 8.4 e 8.5 :

Tabela 8.4: Parâmetros do caso real para tancagem do terminal

\begin{tabular}{|c|c|c|c|}
\hline produto $(p)$ & $C V T I_{p}\left(m^{3}\right)$ & $\operatorname{CVTMIN}_{p}\left(m^{3}\right)$ & $C V T M A X_{p}\left(m^{3}\right)$ \\
\hline 1 & 0 & 0 & 4.000 \\
\hline 2 & 0 & 0 & 4.000 \\
\hline 3 & 22.550 & 0 & 64.465 \\
\hline 4 & 36.913 & 0 & 190.672 \\
\hline
\end{tabular}

Tabela 8.5: Parâmetros do caso real para tancagem do terminal ao longo do tempo

\begin{tabular}{|c|c|c|c|}
\hline produto $(p)$ & instante $(k)$ & $C V T O R_{p, k}\left(m^{3}\right)$ & $C V T O E_{p, k}\left(m^{3}\right)$ \\
\hline 1 & $27 \ldots 34$ & 0 & 2.687 \\
\hline 2 & $5 \ldots 10$ & 0 & 2.687 \\
\hline 2 & $14 \ldots 15$ & 0 & 2.687 \\
\hline 3 & $29 \ldots 33$ & 0 & 3.200 \\
\hline 3 & 34 & 0 & 1.600 \\
\hline 4 & $1 \ldots 5$ & 1.850 & 0 \\
\hline 4 & 6 & 900 & 0 \\
\hline 4 & 7 & 1.250 & 0 \\
\hline 4 & $8 \ldots 11$ & 2.490 & 0 \\
\hline 4 & $23 \ldots 33$ & 1.950 & 0 \\
\hline 4 & $36 \ldots 41$ & 1.625 & 0 \\
\hline 4 & 46 & 800 & 0 \\
\hline 4 & $47 \ldots 50$ & 1.590 & 0 \\
\hline
\end{tabular}

Os parâmetros de volumes relacionados à faixa desejável de estoques estão descritos na tabela 8.6. 
Tabela 8.6: Parâmetros do caso real para a faixa desejável de estoques

\begin{tabular}{|c|c|c|c|c|}
\hline produto $(p)$ & $\begin{array}{c}\text { CVRMIND } \\
\left(\mathrm{m}^{3}\right)\end{array}$ & $\begin{array}{c}\text { CVRMAXD } \\
\left(\mathrm{m}^{3}\right)\end{array}$ & $\begin{array}{c}\text { CVTMIND } \\
\left(\mathrm{m}^{3}\right)\end{array}$ & $\begin{array}{c}\text { CVTMAXD } \\
\left(\mathrm{m}^{3}\right)\end{array}$ \\
\hline 1 & 0 & 6.561 & 0 & 3.200 \\
\hline 2 & 0 & 9.201 & 0 & 3.200 \\
\hline 3 & 12.770 & 42.015 & 16.116 & 32.233 \\
\hline 4 & 28.196 & 205.722 & 32.211 & 47.668 \\
\hline
\end{tabular}

Os parâmetros relativos ao duto que foram utilizados são apresentados na tabela 8.7 (preenchimento inicial do duto). Note que inicialmente o duto é preenchido exclusivamente pelo produto 2 .

Tabela 8.7: Parâmetros para condição inicial do duto

\begin{tabular}{|c|c|c|}
\hline produto $(p)$ & lote $(l)$ & $C X V D I_{l, p}$ \\
\hline 1 & $\mathrm{I} \ldots L$ & 0 \\
\hline 2 & $\mathrm{I} \ldots L$ & 1 \\
\hline 3 & $\mathrm{I} \ldots L$ & 0 \\
\hline 4 & $\mathrm{I} \ldots L$ & 0 \\
\hline
\end{tabular}

Os parâmetros de custo de bombeamento são dados nas tabelas 8.8 e os custos de interface entre os produtos são dados na tabela 8.9.

Tabela 8.8: Parâmetros do caso real para o custo de bombeamento

\begin{tabular}{|c|c|c|}
\hline produto $(p)$ & $\begin{array}{c}C C B R D_{p} \\
\left(\$ / m^{3}\right)\end{array}$ & $\begin{array}{c}C C B T D_{p} \\
\left(\$ / m^{3}\right)\end{array}$ \\
\hline 1 & 1,00 & 1,00 \\
\hline 2 & 1,05 & 1,05 \\
\hline 3 & 1,00 & 1,00 \\
\hline 4 & 1,10 & 1,10 \\
\hline
\end{tabular}

Tabela 8.9: Parâmetros do caso real para o custo da interface

\begin{tabular}{|c|c|c|c|c|}
\hline \multirow{2}{*}{ CIP $_{p, p^{\prime}}$} & \multicolumn{4}{|c|}{ (\$/ocorrência) } \\
\cline { 2 - 5 } & 1 & 2 & 3 & 4 \\
\hline 1 & 0 & 500 & 500 & 500 \\
\hline 2 & 500 & 0 & 500 & 500 \\
\hline 3 & 500 & 500 & 0 & 500 \\
\hline 4 & 500 & 500 & 500 & 0 \\
\hline
\end{tabular}

Os parâmetros de penalização de estoques do caso real são dados na tabela 8.10. 
Tabela 8.10: Parâmetros do caso real para penalização de estoques

\begin{tabular}{|c|c|c|c|c|}
\hline produto $(p)$ & $\begin{array}{c}\text { CPERDLI }_{p} \\
\left(\$ /\left(m^{3} \cdot h\right)\right)\end{array}$ & $\begin{array}{c}\text { CPERDLS }_{p} \\
\left(\$ /\left(m^{3} \cdot h\right)\right)\end{array}$ & $\begin{array}{c}\text { CPETDLI }_{p} \\
\left(\$ /\left(m^{3} \cdot h\right)\right)\end{array}$ & $\begin{array}{c}\text { CPETDLS }_{p} \\
\left(\$ /\left(m^{3} \cdot h\right)\right)\end{array}$ \\
\hline 1 & 5,10 & 5,60 & 5,60 & 5,10 \\
\hline 2 & 5,00 & 5,50 & 5,50 & 5,00 \\
\hline 3 & 5,50 & 5,00 & 5,00 & 5,50 \\
\hline 4 & 5,60 & 5,10 & 5,10 & 5,60 \\
\hline
\end{tabular}

\subsection{RESULTADOS COMPUTACIONAIS}

O sistema computacional utilizado para resolver os modelos de otimização foi o seguinte: GAMS (General Algebraic Modeling System) versão 22.0, utilizando o solver CPLEX versão 10.0.1 com 2 threads, ambos em um microcomputador com 1 processador Intel Dual Core 2 Modelo E6400 - 2.13 GHz, 2 Gb de memória RAM com o sistema operacional Windows $X P$.

A partir dos dados utilizados no item 8.3, o problema tem as seguintes estatísticas:

- 400 variáveis inteiras;

- 5.551 variáveis contínuas;

• 14.129 equações e inequações.

Para este problema real a solução apresentada não corresponde necessariamente à solução ótima do problema, porque o tempo computacional atingiu o limite estipulado, que foi de 36h. A tabela 8.11 quantifica o tempo computacional.

Tabela 8.11: Valores da função objetivo para caso real

\begin{tabular}{|c|c|c|c|}
\hline Tempo Computacional & Iterações & Nós & GAP \\
\hline 36h00min & 245.027 .179 & 2.720 .228 & $48,57 \%$ \\
\hline
\end{tabular}

A tabela 8.12 apresenta a comparação entre os valores da função objetivo da solução real e otimizada. A solução real representa as operações executadas no duto. 
Tabela 8.12: Valores da função objetivo para caso real

\begin{tabular}{|c|c|}
\hline Caso & Valor Função Objetivo \\
\hline "real" & 19.679 .676 \\
\hline Modelo & 13.758 .633 \\
\hline
\end{tabular}

A tabela 8.13 apresenta as movimentações da refinaria para o terminal (o produto que a refinaria envia para dentro do duto) e vice-versa (o produto que o terminal envia para dentro do duto).

Tabela 8.13: Movimentações da refinaria para o terminal e vice-versa para o caso real

\begin{tabular}{|c|c|c|c|c|}
\hline \multirow{2}{*}{ tempo $(k)$} & \multicolumn{2}{|c|}{ refinaria $\rightarrow$ terminal } & \multicolumn{2}{|c|}{ terminal $\rightarrow$ refinaria } \\
\hline & real $(p)$ & modelo $(p)$ & real $(p)$ & modelo $(p)$ \\
\hline 1 & & & & 3 \\
\hline 2 & & & & \\
\hline 3 & & 3 & & \\
\hline 4 & & 3 & & \\
\hline 5 & 4 & 3 & & \\
\hline 6 & 4 & 2 & & \\
\hline 7 & 4 & 2 & & \\
\hline 8 & 4 & 1 & & \\
\hline 9 & 1 & 1 & & \\
\hline 10 & 1 & 1 & & \\
\hline 11 & & 1 & & \\
\hline 12 & & 1 & & \\
\hline 13 & & 2 & & \\
\hline 14 & 1 & 2 & & \\
\hline 15 & 1 & 2 & & \\
\hline 16 & & 2 & & \\
\hline 17 & & & & \\
\hline 18 & & & & \\
\hline 19 & & & & 4 \\
\hline 20 & 1 & & & 4 \\
\hline 21 & 1 & & & 4 \\
\hline 22 & 1 & & & 4 \\
\hline 23 & 1 & 1 & & \\
\hline
\end{tabular}




\begin{tabular}{|c|c|c|c|c|}
\hline \multirow{2}{*}{ tempo $(k)$} & \multicolumn{2}{|c|}{ refinaria $\rightarrow$ terminal } & \multicolumn{2}{|c|}{ terminal $\rightarrow$ refinaria } \\
\hline & real $(p)$ & modelo $(p)$ & real $(p)$ & modelo $(p)$ \\
\hline 24 & & 1 & & \\
\hline 25 & & 1 & & \\
\hline 26 & & 2 & & \\
\hline 27 & 4 & 2 & & \\
\hline 28 & 4 & 2 & & \\
\hline 29 & 4 & 2 & & \\
\hline 30 & 4 & 2 & & \\
\hline 31 & 4 & 2 & & \\
\hline 32 & 4 & 2 & & \\
\hline 33 & 4 & 2 & & \\
\hline 34 & 4 & & & 4 \\
\hline 35 & & & 4 & 4 \\
\hline 36 & & & 4 & 4 \\
\hline 37 & & & 4 & 4 \\
\hline 38 & & & 4 & 4 \\
\hline 39 & & & 4 & 4 \\
\hline 40 & & & 4 & 4 \\
\hline 41 & & & 4 & 4 \\
\hline 42 & & & 4 & 4 \\
\hline 43 & & & 4 & 4 \\
\hline 44 & & & 4 & 4 \\
\hline 45 & & & 4 & 4 \\
\hline 46 & & & 4 & 4 \\
\hline 47 & & & 4 & 4 \\
\hline 48 & & & 4 & 4 \\
\hline 49 & & & 4 & 4 \\
\hline 50 & & & 4 & 4 \\
\hline
\end{tabular}

Os perfis do conteúdo do duto ao longo do horizonte do tempo estão apresentados na tabela 8.14. 
Tabela 8.14: Perfis do duto no caso real e MPE

\begin{tabular}{|c|c|c|c|c|c|c|c|c|c|c|c|c|c|c|c|c|c|c|c|}
\hline \multirow{2}{*}{$\begin{array}{c}\text { Tempo } \\
(k)\end{array}$} & \multicolumn{9}{|c|}{ Lotes - Real } & \multicolumn{10}{|c|}{ Lotes - Modelo } \\
\hline & & 1 & 2 & 3 & 4 & 5 & 6 & 7 & 8 & & 1 & 2 & 3 & 4 & 5 & 6 & 7 & 8 & \\
\hline 1 & & 2 & 2 & 2 & 2 & 2 & 2 & 2 & 2 & & 2 & 2 & 2 & 2 & 2 & 2 & 2 & 3 & $\leftarrow$ \\
\hline 2 & & 2 & 2 & 2 & 2 & 2 & 2 & 2 & 2 & & 2 & 2 & 2 & 2 & 2 & 2 & 2 & 3 & \\
\hline 3 & & 2 & 2 & 2 & 2 & 2 & 2 & 2 & 2 & $\rightarrow$ & 3 & 2 & 2 & 2 & 2 & 2 & 2 & 2 & \\
\hline 4 & & 2 & 2 & 2 & 2 & 2 & 2 & 2 & 2 & $\rightarrow$ & 3 & 3 & 2 & 2 & 2 & 2 & 2 & 2 & \\
\hline 5 & $\rightarrow$ & 4 & 2 & 2 & 2 & 2 & 2 & 2 & 2 & $\rightarrow$ & 3 & 3 & 3 & 2 & 2 & 2 & 2 & 2 & \\
\hline 6 & $\rightarrow$ & 4 & 4 & 2 & 2 & 2 & 2 & 2 & 2 & $\rightarrow$ & 2 & 3 & 3 & 3 & 2 & 2 & 2 & 2 & \\
\hline 7 & $\rightarrow$ & 4 & 4 & 4 & 2 & 2 & 2 & 2 & 2 & $\rightarrow$ & 2 & 2 & 3 & 3 & 3 & 2 & 2 & 2 & \\
\hline 8 & $\rightarrow$ & 4 & 4 & 4 & 4 & 2 & 2 & 2 & 2 & $\rightarrow$ & 1 & 2 & 2 & 3 & 3 & 3 & 2 & 2 & \\
\hline 9 & $\rightarrow$ & 1 & 4 & 4 & 4 & 4 & 2 & 2 & 2 & $\rightarrow$ & 1 & 1 & 2 & 2 & 3 & 3 & 3 & 2 & \\
\hline 10 & $\rightarrow$ & 1 & 1 & 4 & 4 & 4 & 4 & 2 & 2 & $\rightarrow$ & 1 & 1 & 1 & 2 & 2 & 3 & 3 & 3 & \\
\hline 11 & & 1 & 1 & 4 & 4 & 4 & 4 & 2 & 2 & $\rightarrow$ & 1 & 1 & 1 & 1 & 2 & 2 & 3 & 3 & \\
\hline 12 & & 1 & 1 & 4 & 4 & 4 & 4 & 2 & 2 & $\rightarrow$ & 1 & 1 & 1 & 1 & 1 & 2 & 2 & 3 & \\
\hline 13 & & 1 & 1 & 4 & 4 & 4 & 4 & 2 & 2 & $\rightarrow$ & 2 & 1 & 1 & 1 & 1 & 1 & 2 & 2 & \\
\hline 14 & $\rightarrow$ & 1 & 1 & 1 & 4 & 4 & 4 & 4 & 2 & $\rightarrow$ & 2 & 2 & 1 & 1 & 1 & 1 & 1 & 2 & \\
\hline 15 & $\rightarrow$ & 1 & 1 & 1 & 1 & 4 & 4 & 4 & 4 & $\rightarrow$ & 2 & 2 & 2 & 1 & 1 & 1 & 1 & 1 & \\
\hline 16 & & 1 & 1 & 1 & 1 & 4 & 4 & 4 & 4 & $\rightarrow$ & 2 & 2 & 2 & 2 & 1 & 1 & 1 & 1 & \\
\hline 17 & & 1 & 1 & 1 & 1 & 4 & 4 & 4 & 4 & & 2 & 2 & 2 & 2 & 1 & 1 & 1 & 1 & \\
\hline 18 & & 1 & 1 & 1 & 1 & 4 & 4 & 4 & 4 & & 2 & 2 & 2 & 2 & 1 & 1 & 1 & 1 & \\
\hline 19 & & 1 & 1 & 1 & 1 & 4 & 4 & 4 & 4 & & 2 & 2 & 2 & 1 & 1 & 1 & 1 & 4 & $\leftarrow$ \\
\hline 20 & $\rightarrow$ & 1 & 1 & 1 & 1 & 1 & 4 & 4 & 4 & & 2 & 2 & 1 & 1 & 1 & 1 & 4 & 4 & $\leftarrow$ \\
\hline 21 & $\rightarrow$ & 1 & 1 & 1 & 1 & 1 & 1 & 4 & 4 & & 2 & 1 & 1 & 1 & 1 & 4 & 4 & 4 & $\leftarrow$ \\
\hline 22 & $\rightarrow$ & 1 & 1 & 1 & 1 & 1 & 1 & 1 & 4 & & 1 & 1 & 1 & 1 & 4 & 4 & 4 & 4 & $\leftarrow$ \\
\hline 23 & $\rightarrow$ & 1 & 1 & 1 & 1 & 1 & 1 & 1 & 1 & $\rightarrow$ & 1 & 1 & 1 & 1 & 1 & 4 & 4 & 4 & \\
\hline 24 & & 1 & 1 & 1 & 1 & 1 & 1 & 1 & 1 & $\rightarrow$ & 1 & 1 & 1 & 1 & 1 & 1 & 4 & 4 & \\
\hline 25 & & 1 & 1 & 1 & 1 & 1 & 1 & 1 & 1 & $\rightarrow$ & 1 & 1 & 1 & 1 & 1 & 1 & 1 & 4 & \\
\hline 26 & & 1 & 1 & 1 & 1 & 1 & 1 & 1 & 1 & $\rightarrow$ & 2 & 1 & 1 & 1 & 1 & 1 & 1 & 1 & \\
\hline 27 & $\rightarrow$ & 4 & 1 & 1 & 1 & 1 & 1 & 1 & 1 & $\rightarrow$ & 2 & 2 & 1 & 1 & 1 & 1 & 1 & 1 & \\
\hline 28 & $\rightarrow$ & 4 & 4 & 1 & 1 & 1 & 1 & 1 & 1 & $\rightarrow$ & 2 & 2 & 2 & 1 & 1 & 1 & 1 & 1 & \\
\hline 29 & $\rightarrow$ & 4 & 4 & 4 & 1 & 1 & 1 & 1 & 1 & $\rightarrow$ & 2 & 2 & 2 & 2 & 1 & 1 & 1 & 1 & \\
\hline
\end{tabular}




\begin{tabular}{|c|c|c|c|c|c|c|c|c|c|c|c|c|c|c|c|c|c|c|c|c|}
\hline Tempo & \multicolumn{10}{|c|}{ Lotes - Real } & \multicolumn{10}{|c|}{ Lotes - Modelo } \\
\hline$(k)$ & & 1 & 2 & 3 & 4 & 5 & 6 & 7 & 8 & & & 1 & 2 & 3 & 4 & 5 & 6 & 7 & 8 & \\
\hline 30 & $\rightarrow$ & 4 & 4 & 4 & 4 & 1 & 1 & 1 & 1 & & $\rightarrow$ & 2 & 2 & 2 & 2 & 2 & 1 & 1 & 1 & \\
\hline 31 & $\rightarrow$ & 4 & 4 & 4 & 4 & 4 & 1 & 1 & 1 & & $\rightarrow$ & 2 & 2 & 2 & 2 & 2 & 2 & 1 & 1 & \\
\hline 32 & $\rightarrow$ & 4 & 4 & 4 & 4 & 4 & 4 & 1 & 1 & & $\rightarrow$ & 2 & 2 & 2 & 2 & 2 & 2 & 2 & 1 & \\
\hline 33 & $\rightarrow$ & 4 & 4 & 4 & 4 & 4 & 4 & 4 & 1 & & $\rightarrow$ & 2 & 2 & 2 & 2 & 2 & 2 & 2 & 2 & \\
\hline 34 & $\rightarrow$ & 4 & 4 & 4 & 4 & 4 & 4 & 4 & 4 & & & 2 & 2 & 2 & 2 & 2 & 2 & 2 & 4 & $\leftarrow$ \\
\hline 35 & & 4 & 4 & 4 & 4 & 4 & 4 & 4 & 4 & $\leftarrow$ & & 2 & 2 & 2 & 2 & 2 & 2 & 4 & 4 & $\leftarrow$ \\
\hline 36 & & 4 & 4 & 4 & 4 & 4 & 4 & 4 & 4 & $\leftarrow$ & & 2 & 2 & 2 & 2 & 2 & 4 & 4 & 4 & $\leftarrow$ \\
\hline 37 & & 4 & 4 & 4 & 4 & 4 & 4 & 4 & 4 & $\leftarrow$ & & 2 & 2 & 2 & 2 & 4 & 4 & 4 & 4 & $\leftarrow$ \\
\hline 38 & & 4 & 4 & 1 & 4 & 4 & 4 & 4 & 1 & $\leftarrow$ & & 2 & 2 & 2 & 4 & 4 & 4 & 4 & 4 & $\leftarrow$ \\
\hline 39 & & 4 & 4 & 4 & 4 & 4 & 4 & 4 & 4 & $\leftarrow$ & & 2 & 2 & 4 & 4 & 4 & 4 & 4 & 4 & $\leftarrow$ \\
\hline 40 & & 4 & 4 & 4 & 4 & 4 & 4 & 4 & 4 & $\leftarrow$ & & 2 & 4 & 4 & 4 & 4 & 4 & 4 & 4 & $\leftarrow$ \\
\hline 41 & & 4 & 4 & 4 & 4 & 4 & 4 & 4 & 4 & $\leftarrow$ & & 4 & 4 & 4 & 4 & 4 & 4 & 4 & 4 & $\leftarrow$ \\
\hline 42 & & 4 & 4 & 4 & 4 & 4 & 4 & 4 & 4 & $\leftarrow$ & & 4 & 4 & 4 & 4 & 4 & 4 & 4 & 4 & $\leftarrow$ \\
\hline 43 & & 4 & 4 & 4 & 4 & 4 & 4 & 4 & 4 & $\leftarrow$ & & 4 & 4 & 4 & 4 & 4 & 4 & 4 & 4 & $\leftarrow$ \\
\hline 44 & & 4 & 4 & 4 & 4 & 4 & 4 & 4 & 4 & $\leftarrow$ & & 4 & 4 & 4 & 4 & 4 & 4 & 4 & 4 & $\leftarrow$ \\
\hline 45 & & 4 & 4 & 4 & 4 & 4 & 4 & 4 & 4 & $\leftarrow$ & & 4 & 4 & 4 & 4 & 4 & 4 & 4 & 4 & $\leftarrow$ \\
\hline 46 & & 4 & 4 & 4 & 4 & 4 & 4 & 4 & 4 & $\leftarrow$ & & 4 & 4 & 4 & 4 & 4 & 4 & 4 & 4 & $\leftarrow$ \\
\hline 47 & & 4 & 4 & 4 & 4 & 4 & 4 & 4 & 4 & $\leftarrow$ & & 4 & 4 & 4 & 4 & 4 & 4 & 4 & 4 & $\leftarrow$ \\
\hline 48 & & 4 & 4 & 4 & 4 & 4 & 4 & 4 & 4 & $\leftarrow$ & & 4 & 4 & 4 & 4 & 4 & 4 & 4 & 4 & $\leftarrow$ \\
\hline 49 & & 4 & 4 & 4 & 4 & 4 & 4 & 4 & 4 & $\leftarrow$ & & 4 & 4 & 4 & 4 & 4 & 4 & 4 & 4 & $\leftarrow$ \\
\hline 50 & & 4 & 4 & 4 & 4 & 4 & 4 & 4 & 4 & $\leftarrow$ & & 4 & 4 & 4 & 4 & 4 & 4 & 4 & 4 & $\leftarrow$ \\
\hline
\end{tabular}

As figuras 8.2, 8.3, 8.4 e 8.5 apresentam os estoques na refinaria e no terminal ao longo do tempo dos produtos 1, 2, 3 e 4 repesctivamente. 


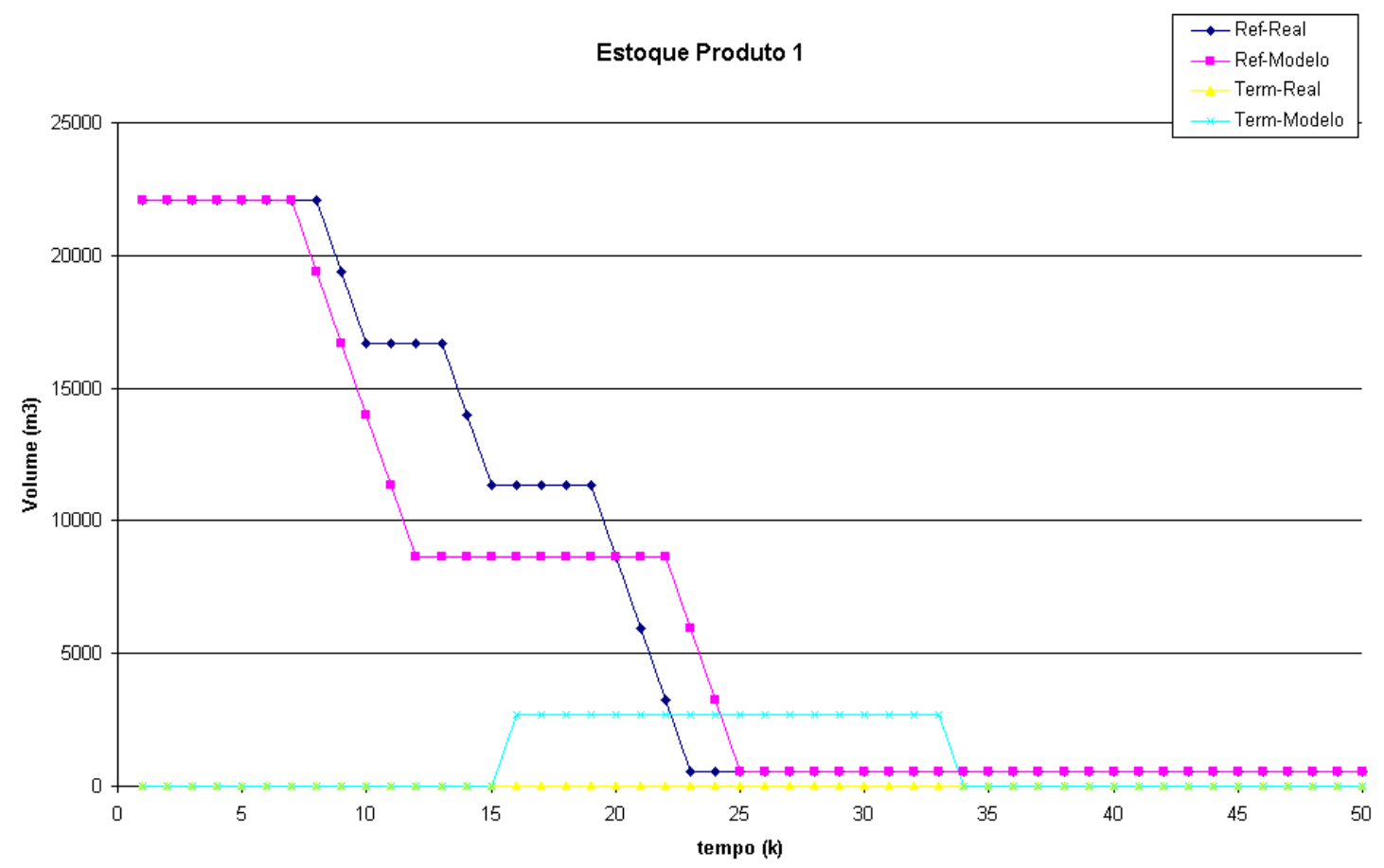

Figura 8.2: Estoque do produto $p=1$ na refinaria e no terminal no caso real

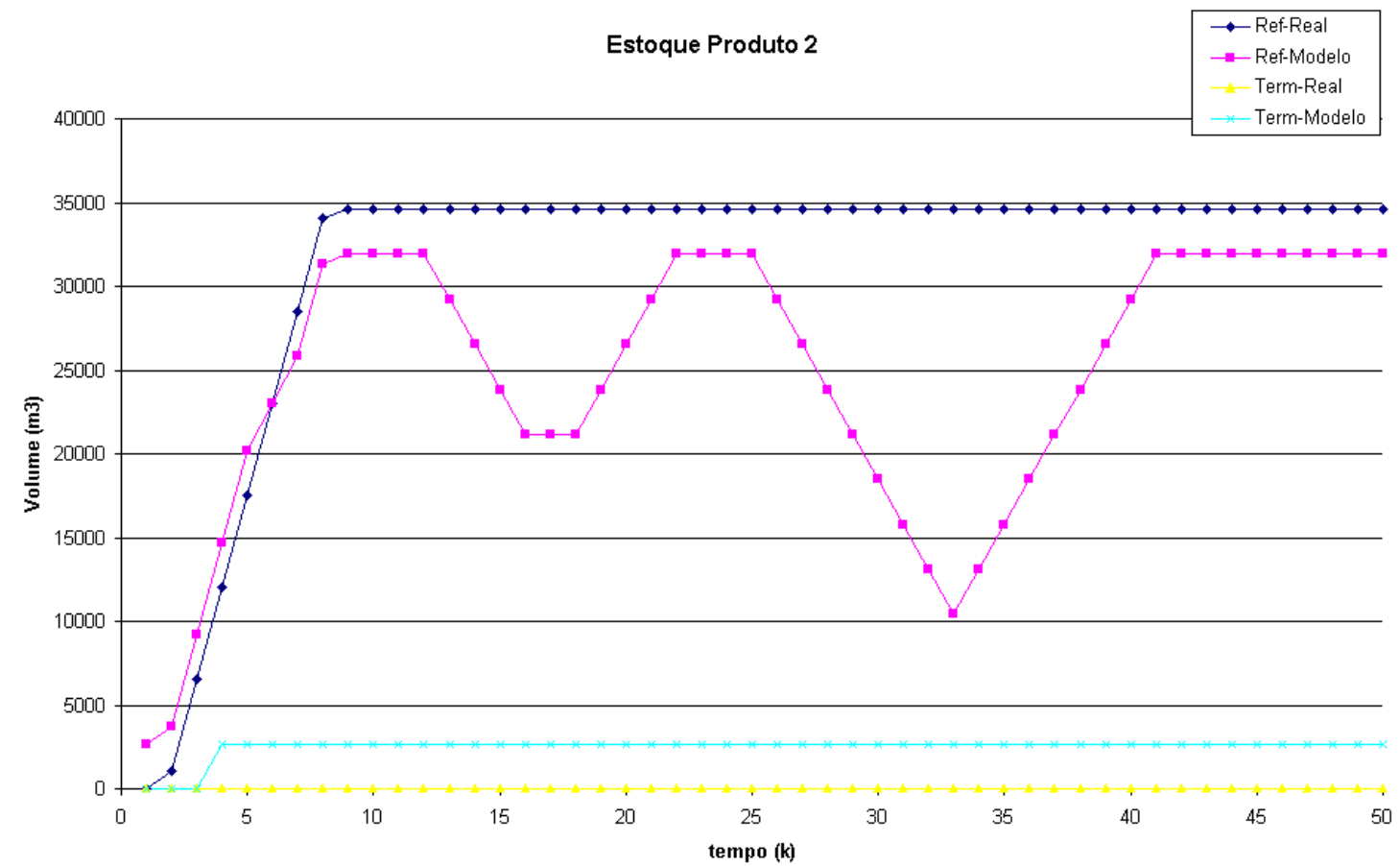

Figura 8.3: Estoque do produto $p=2$ na refinaria e no terminal no caso real 


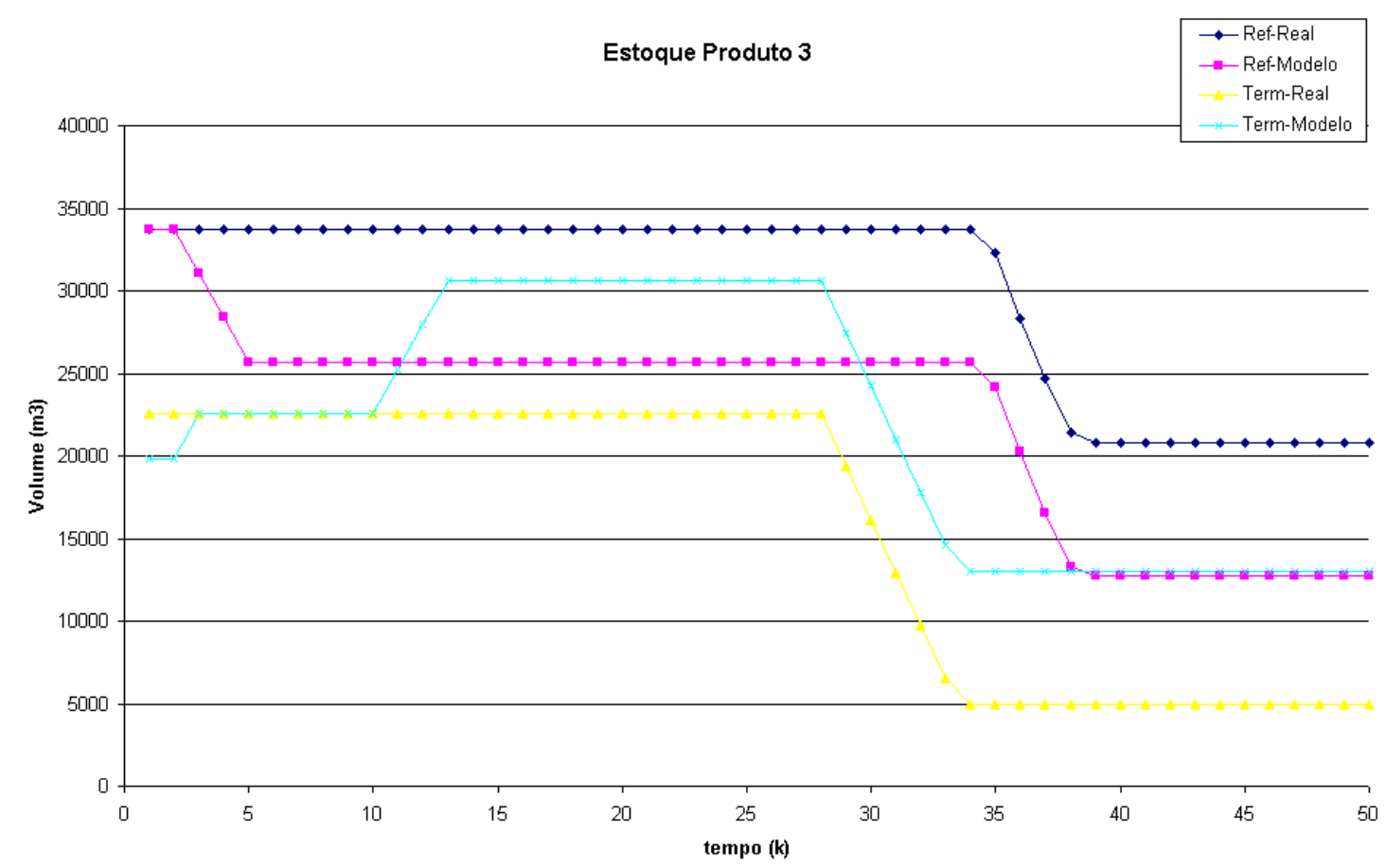

Figura 8.4: Estoque do produto $p=3$ na refinaria e no terminal no caso real

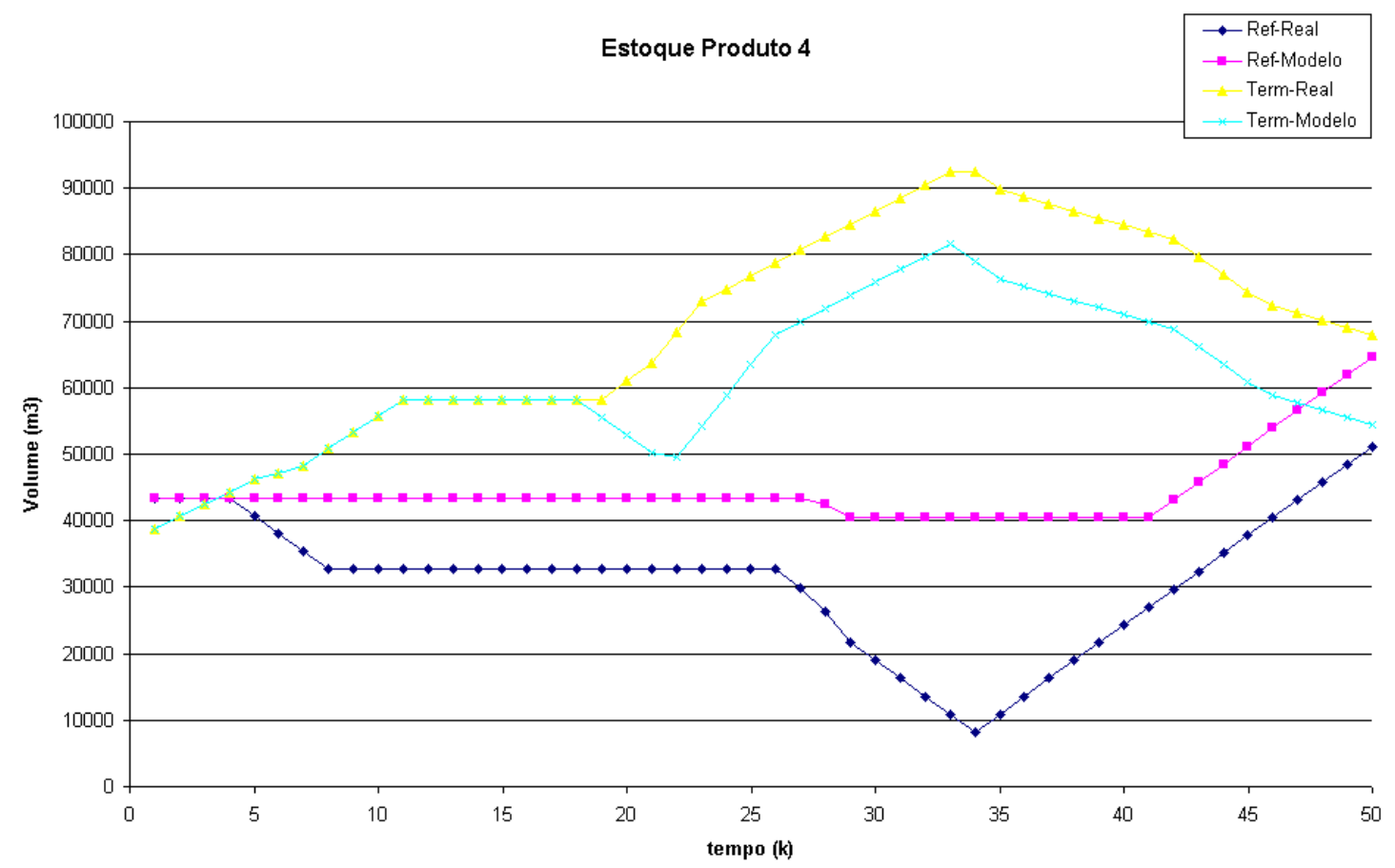

Figura 8.5: Estoque do produto $p=4$ na refinaria e no terminal no caso real 


\subsection{DISCUSSÃO DOS RESULTADOS}

A redução da função objetivo em relação à solução manual foi de $30 \%$. A diferença entre o valor da solução manual com a do modelo em grande parte do valor é obtido nas penalizões dos estoques. Isto ocorreu com o estoque do produto 2 na refinaria, onde em ambas as soluções tiveram as penalizações, mas a diferença em relação ao limite máximo desejável $\left(C V R M A X D_{p=2}=9.201\right)$ foi menor para o modelo. Para o produto 3 ambas as soluções também tiveram penalizações no estoque do terminal e o mesmo comportamento das soluções foi verificado. Para o produto 4, o estoque na refinaria ficou abaixo do limite mínimo desejável $\left(C V R M I N D_{p=4}=28.196\right)$ na solução manual, entretanto na solução do modelo isto não ocorreu. Para este último produto, ambas as soluções penalizam os estoques do terminal, mas a diferença entre o limite máximo desejável $\left(C V T M A X D_{p=4}=47.668\right)$ e a solução do modelo é menor que a correspondente na solução manual.

Verifica-se que o sistema enviou o produto 3 da refinaria para o terminal no primeiro intervalo, e parou no instante seguinte. A partir do $3^{\circ}$ até $016^{\circ}$ intervalo 0 sistema movimentou da refinaria para o terminal, sendo o produto 3 do $3^{\circ}$ ao $5^{\circ}$, 0 produto 2 no $6^{\circ}$ e $7^{\circ}$, produto 1 no $8^{\circ}$ ao $12^{\circ}$ e o produto 2 no $13^{\circ}$ ao $16^{\circ}$ intervalo. Nos 2 intervalos seguintes há uma parada no duto, em seguida há uma inversão de sentido do duto até o $22^{\circ}$ intervalo com o produto 4 . Na seqüência há novamente uma inversão do duto (refinaria $\rightarrow$ terminal) até o $33^{\circ}$ intervalo, sendo o produto 1 do $23^{\circ}$ ao $25^{\circ}$ e o produto 2 do $26^{\circ}$ ao $33^{\circ}$. Há novamente uma inversão de sentido no duto, este movimentando o produto 4 até o último intervalo.

Na solução viável encontrada (não é a solução ótima), verificam-se diversas motivações para algumas das operações. Para atender a demanda do produto 2 no terminal (parâmetro $C V T O E_{p, k}$ ) o duto é movimentado do $3^{\circ}$ ao $16^{\circ}$ intervalo no sentido refinaria $\rightarrow$ terminal. Para atender a demanda do produto 1 no terminal o duto é também movimentado do $23^{\circ}$ ao $33^{\circ}$ intervalo no sentido da refinaria para o terminal. As movimentações do produto 4 do terminal para a refinaria buscam diminuir a penalização do produto 4 no terminal (quase todo os intervalos o produto 4 encontra-se fora da faixa desejável). 


\section{CONCLUSÕES E PROPOSTAS DE CONTINUAÇÃO DO TRABALHO}

\subsection{PRINCIPAIS CONTRIBUIÇÕES DO TRABALHO}

Este trabalho aborda o problema de programação de sistemas compostos de dutos com operação bidirecional. Tais sistemas ocorrem com freqüência em malhas dutoviárias reais e até o momento sua operação não foi considerada com a abordagem de otimização. O objetivo foi desenvolver uma formulação de um modelo de otimização de forma genérica e verificar a sua aplicabilidade a problemas reais. O modelo desenvolvido é composto por variáveis binárias e contínuas, e por restrições lineares. As principais contribuições deste trabalho são as seguintes:

- Revisão da literatura sobre programação de produção, planejamento e gerenciamento da cadeia de suprimento da indústria de petróleo e em particular em sistemas dutoviários;

- Descrição detalhada do problema com ênfase nas aplicações reais de sistemas de malhas dutoviárias;

- Desenvolvimento de um modelo MILP para a programação de dutos com operação bidirecional;

- Aplicação do modelo a um sistema protótipo e comparação da solução otimizada com uma solução manual;

- Desenvolvimento de restrições de cortes para aumento da eficiência computacional do modelo;

- Formulação alternativa da função objetivo que penaliza estoques fora da faixa de operação; 
- Aplicação do modelo a um sistema real de transporte de múltiplos produtos.

\subsection{SUMÁRIO DA DISSERTAÇÃO}

O capítulo 2 apresenta uma revisão da literatura que aborda os aspectos e características da programação de produção, planejamento e gerenciamento da cadeia de suprimento na indústria de petróleo e em particular em sistemas dutoviários. São apresentados os diferentes tipos de algoritmos para resolução de cada tipo de problema, como modelos de programação matemática (MILP, MINLP), meta-heurísticas (CLP), heurísticas (IA) e respectivas combinações.

Uma descrição do sistema de malha de dutos reais operada pela Transpetro S.A. é apresentada no capítulo 3, o qual também descreve o problema de programação da malha de dutos com as suas características. É descrito de forma detalhada o problema de inversão de sentido do duto, com ênfase nas aplicações reais;

Baseado no trabalho desenvolvido por Rejowski Jr (2001), no capítulo 4 é desenvolvido um modelo de programação matemática mista inteira (MILP), que tem como principais características a divisão do duto em lotes de tamanho fixo e vazão do duto fixa, que resultam em uma representação do tempo de forma discreta; balanço de material na refinaria, duto e terminal; operação bidirecional do duto e algumas restrições do modelo de negócio.

No capítulo 5 foi aplicado o modelo desenvolvido a um sistema protótipo, no qual há de necessidade de reversão do duto. A solução ótima encontrada pelo modelo foi comparada a uma solução manual. Nas discussões dos resultados verifica-se que o parâmetro custo de estocagem tem uma influência decisiva na busca da solução ótima.

Objetivando do aumento da eficiência computacional do modelo baseado em Rejowski Jr e Pinto (2003) e em Rejowski Jr e Pinto (2004), no capítulo 6 desenvolveramse restrições de cortes válidos que reduzem a região viável do modelo relaxado sem modificar a região viável inteira do mesmo. Foram ainda propostas formulações aplicadas para cada instante de tempo. Avaliou a redução do tempo computacional de diferentes modelos que contém os cortes válidos.

Devido à não aderência da solução encontrada pelo modelo a casos reais, uma formulação alternativa da função objetivo que penaliza estoques fora de uma faixa 
de operação foi apresentada no capítulo 7, tendo como foco representar melhor a lógica do programador de dutos. Um aspecto interessante foi a melhora do tempo computacional obtida para o caso protótipo.

Aplicação do modelo reformulado com as restrições de cortes a um sistema real observou que o tempo computacional para obter a solução otimizada ainda é bastante elevada. Uma provável causa desta demora é a degeneração do modelo.

\subsection{PROPOSTAS DE CONTINUAÇÃO DO TRABALHO}

A continuidade do trabalho efetuado até o momento requer os seguintes desenvolvimentos:

\section{- Armazenagem não agregada}

A hipótese H4.19 não passa a ser mais válida, e cada unidade de armazenamento está representada no modelo;

\section{- Formulação do tempo contínuo}

Reformulação deste modelo para o domínio do tempo contínuo. A formulação apresentada na dissertação é de forma discreta com tamanhos iguais e fixos dos lotes do duto. Uma formulação que pode ser adaptada é a desenvolvida por Moro e Pinto (2004) para o tempo contínuo;

\section{- Integração com outros modelos}

Integração com modelos de scheduling de refinaria e distribuição;

\section{- Algoritmo híbrido de solução do MILP}

Utilização de outras técnicas para busca de uma solução inteira viável, gerando um limite para a busca utilizando o modelo MILP. Uma destas técnicas seria a programação por restrição (CLP) que alcançaria uma solução inteira viável e a partir daí seria utilizado o algoritmo de Branch\&Bound (B\&B) para resolver o modelo MILP. A motivação desta estratégia de solução seria uma melhora no tempo computacional na busca de uma solução ótima;

\section{- Algoritmo com restrições de cortes}

Utilização de técnicas similares às desenvolvidas no capítulo 6. Porém neste caso ao invés de gerar cortes válidos, gerar cortes para verificar se existe solução dentro de limites dos volumes descritos no capítulo 7. A motivação desta 
estratégia seria uma melhora na busca de uma solução para sistemas que apresentem "folgas" como o problema real apresentado. 


\section{REFERÊNCIAS}

ASSIS, E. L. B. de. Otimização das operações de distribuição de uma malha dutoviária. 111 p. Dissertação (Mestrado) - Escola Politécnica da USP, São Paulo, jun 2002.

BANKES, W. F. Automated system for scheduling pipeline time for small batch production using a symphony spreadsheet. Computers \& Industrial Engineering, v. 14, n. 1-4, p. 303-307, 1986. Disponível em: $<$ http://dx.doi.org/10.1016/0360-8352(86)90099-9>.

BIEGLER, L. T.; GROSSMANN, I. E.; WESTERBERG, A. W. Systematic Methods of Chemical Process Design. 1. ed. New York: Prentice Hall PTR, 1997. ISBN 0134924223.

BROOKE, A.; KENDRICK, D.; MEERAUS, A.; RAMAN, R. GAMS, A Users Guide. Washington, DC, 2005.

CAFARO, D. C.; CERDÁ, J. Optimal scheduling of multiproduct pipeline systems using a non-discrete milp formulation. Computers \& Chemical Engineering, v. 28, n. 10, p. 2053-2068, set 2004. Disponível em: $<$ http://dx.doi.org/10.1016/j.compchemeng.2004.03.010 >.

CASAS-LIZA, J.; PINTO, J. M. Optimal scheduling of a lube oil and paraffin production plant. Computers \& Chemical Engineering, v. 29, n. 6, p. 1329-1344, mai 2005. Disponível em: <http://dx.doi.org/10.1016/j.compchemeng.2005.02.032>.

ESCUDERO, L. F.; QUINTANA, F. J.; SALMERÓN, J. Coro, a modeling and an algorithmic framework for oil supply, transformation and distribution optimization under uncertainty. European Journal of Operational Research, v. 114, n. 3, p. 638-656, mai 1999. Disponível em: <http://dx.doi.org/10.1016/S03772217(98)00261-6>.

GAMS DEVELOPMENT CORPORATION. GAMS, The Solver Manual. Washington, DC, 2005.

GÖTHE-LUNDGREN, M.; LUNDGREN, J. T.; PERSSON, J. A. An optimization model for refinery production scheduling. International Journal of Production Economics, v. 78, n. 3, p. 255-270, ago 2002. Disponível em: $<$ http://dx.doi.org/10.1016/S0925-5273(00)00162-6>.

HANE, C. A.; RATLIFF, H. D. Sequencing inputs to multi-commodity pipelines. Annals of Operations Research, Springer Netherlands, v. 57, n. 1, p. 73-101, dez 1995. ISSN 0254-5330 (Print) 1572-9338 (Online). Disponível em: $<$ http://dx.doi.org/10.1007/BF02099692>. 
JOLY, M. Técnicas de otimização mista-inteira para o scheduling e gerenciamento da produção em refinarias de petróleo. 195 p. Dissertação (Mestrado) — Escola Politécnica da USP, São Paulo, ago 1999.

JOLY, M.; MORO, L. F. L.; PINTO, J. M. Planning and scheduling for petroleum refineries using mathematical programming. Brazilian Journal of Chemical Engineering, v. 19, n. 2, p. 207-228, abr 2002. ISSN 0104-6632. Disponível em: $<$ http://dx.doi.org/10.1590/S0104-66322002000200008>.

JOLY, M.; PINTO, J. M. Mixed-integer programming techniques for the scheduling of fuel oil and asphalt production. Chemical Engineering Research \& Design, v. 81, n. A4, p. 427-447, abr 2003. ISSN 0263-8762(print) 1744-3563(on-line). Disponível em: <http://dx.doi.org/10.1205/026387603765173691>.

LEE, H.; PINTO, J. M.; GROSSMANN, I. E.; PARK, S. Mixed-integer linear programming model for refinery short-term scheduling of crude oil unloading with inventory management. Industrial \& Engineering Chemistry Research, v. 35, n. 5, p. 1630-1641, 1996. Disponível em: <http://dx.doi.org/10.1021/ie950519h>.

MAGALHÃES, M. V. de O. Refinery Scheduling. 273 p. Tese (Doutorado) - Imperial College London, Londres, jul 2004.

MAGALHÃES, M. V. de O.; MORO, L. F. L.; FILHO SMANIA, P.; HASSIMOTTO, M. K.; PINTO, J. M.; ABADIA, G. J. Sipp - a solution for refinery scheduling. In: NPRA Computer Conference. San Antonio, Texas: [s.n.], 1998. CC-98-145.

MAGATÃO, L. Mixed Integer Linear Programming and Constraint Logic Programming: Towards a Unified Modeling Framework. 186 p. Tese (Doutorado) - Centro Federal de Educação Tecnológica do Paraná, Paraná, maio 2005.

MAGATÃO, L.; ARRUDA, L. V. R.; NEVES, J. F. A mixed integer programming approach for scheduling commodities in a pipeline. Computers \& Chemical Engineering, v. 28, n. 1-2, p. 171-185, jan 2004. Disponível em: $<$ http://dx.doi.org/10.1016/S0098-1354(03)00165-0>.

MÁS, R.; PINTO, J. M. A mixed-integer optimization strategy for oil supply in distribution complexes. Optimization and Engineering, Springer Netherlands, v. 4, n. 1-2, p. 23-64, jun 2003. ISSN 1389-4420 (Print) 1573-2924 (Online). Disponível em: <http://dx.doi.org/10.1023/A:1021808313306>.

MORO, L. F. L. Técnicas de otimização mista inteira para o planejamento e programação de produção em refinarias de petróleo. 182 p. Tese (Doutorado) — Escola Politécnica da USP, São Paulo, dez 2000.

MORO, L. F. L.; PINTO, J. M. Mixed-integer programming approach for short-term crude oil scheduling. Industrial \& Engineering Chemistry Research, v. 43, n. 1, p. 85-94, 2004. Disponível em: <http://dx.doi.org/10.1021/ie030348d>.

MORO, L. F. L.; ZANIN, A. C.; PINTO, J. M. A planning model for refinery diesel production. Computers \& Chemical Engineering, v. 22, n. 1, p. 1039-1042, mar 1998. Disponível em: <http://dx.doi.org/10.1016/S0098-1354(98)00209-9>. 
NEIRO, S. M. S.; PINTO, J. M. A general modeling framework for the operational planning of petroleum supply chains. Computers \& Chemical Engineering, v. 28, n. 6-7, p. 871-896, jun 2004. Disponível em: $<$ http://dx.doi.org/10.1016/j.compchemeng.2003.09.018>.

PETROBRAS S.A. Capacidade das refinarias. Rio de Janeiro. Seção: Negócios/Downstream/Refinarias. Disponível em: <http://www.petrobras.com.br/>. Acesso em: 06 maio 2005.

PINTO, J. M.; GROSSMANN, I. E. Assignment and sequencing models for the scheduling of process systems. Annals of Operations Research, Springer Netherlands, v. 81, p. 433-466, jun 1998. ISSN 0254-5330 (Print) 1572-9338 (Online). Disponível em: <http://dx.doi.org/10.1023/A:1018929829086>.

PINTO, J. M.; JOLY, M. Mixed-integer programming techniques for the scheduling of fuel oil and asphalt production. In: ESCAPE 10 - European Symposium on Computer-Aided Process Engineering. Florença: [s.n.], 2000. v. 8, p. 1063-1068.

PINTO, J. M.; JOLY, M.; MORO, L. F. L. Planning and scheduling models for refinery operations. Computers \& Chemical Engineering, v. 24, n. 9-10, p. 2259-2276, out 2000. Disponível em: <http://dx.doi.org/10.1016/S0098-1354(00)00571-8>.

PINTO, J. M.; MORO, L. F. L. A mixed-integer model for Ipg scheduling. In: ESCAPE 10 - European Symposium on Computer-Aided Process Engineering. Florença: [s.n.], 2000. v. 8, p. 1141-1146.

PINTO, J. M.; TURKAY, A.; BIOLO, B.; GROSSMANN, I. E. Stbs: A continuous-time milp optimization for short-term scheduling of batch plants. Computers \& Chemical Engineering, v. 22, n. 9, p. 1297-1308, 1998. Disponível em: $<$ http://dx.doi.org/10.1016/S0098-1354(98)00014-3>.

PIRKUL, H.; JAYARAMAN, V. A multi-commodity, multi-plant, capacitated facility location problem: formulation and efficient heuristic solution. Computers And Operations Research, v. 25, n. 10, p. 869-878, out 1998. Disponível em: $<$ http://dx.doi.org/10.1016/S0305-0548(97)00096-8>.

PONGSAKDI, A.; RANGSUNVIGIT, P.; SIEMANOND, K.; BAGAJEWICZ, M. J. Financial risk management in the planning of refinery operations. International Journal of Production Economics, v. 103, n. 1, p. 64-86, set 2006. Disponível em: <http://dx.doi.org/10.1016/j.ijpe.2005.04.007>.

RAMAN, R.; GROSSMANN, I. E. Relation between milp modelling and logical inference for chemical process synthesis. Computers \& Chemical Engineering, v. 15, n. 2, p. 73-84, fev 1991. Disponível em: < http://dx.doi.org/10.1016/00981354(91)87007-V>.

RAMAN, R.; GROSSMANN, I. E. Modelling and computational techniques for logic based integer programming. Computers \& Chemical Engineering, v. 18, n. 7, p. 563-578, jul 1994. Disponível em: <http://dx.doi.org/10.1016/00981354(93)E0010-7>. 
REJOWSKI JR, R. Programação de distribuição dutoviária de derivados de petróleo. 145 p. Dissertação (Mestrado) — Escola Politécnica da USP, São Paulo, set 2001.

REJOWSKI JR, R.; PINTO, J. M. An milp formulation for the scheduling of multiproduct pipeline system. Brazilian Journal of Chemical Engineering, v. 19, n. 4, p. 467-474, out 2002. ISSN 0104-6632.

REJOWSKI JR, R.; PINTO, J. M. Scheduling of a multiproduct pipeline system. Computers and Chemical Engineering, v. 27, n. 8-9, p. 1229-1246, set 2003. Disponível em: <http://dx.doi.org/10.1016/S0098-1354(03)00049-8>.

REJOWSKI JR, R.; PINTO, J. M. Efficient milp formulations and valid cuts for multiproduct pipeline scheduling. Computers \& Chemical Engineering, v. 28, p. 1511-1528, 2004. Disponível em: $<$ http://dx.doi.org/10.1016/j.compchemeng.2003.12.001>.

SASIKUMAR, M.; PRAKASH, P. R.; PATIL, S. M.; RAMANI, S. Pipes: A heuristic search model for pipeline schedule generation. KnowledgeBased Systems, v. 10, n. 3, p. 169-175, out 1997. Disponível em: $<$ http://dx.doi.org/10.1016/S0950-7051(97)00026-9>.

SAWAYA, N. W.; GROSSMANN, I. E. A cutting plane method for solving linear generalized disjunctive programming problems. Computers \& Chemical Engineering, v. 29, n. 9, p. 1891-1913, ago 2005. Disponível em: $<$ http://dx.doi.org/10.1016/j.compchemeng.2005.04.004>.

$\mathrm{SHAH}, \mathrm{N}$. Mathematical programming techniques for crude oil scheduling. Computers \& Chemical Engineering, v. 20, n. 2, p. S1227-S1232, 1996. Disponível em: $<$ http://dx.doi.org/10.1016/0098-1354(96)00212-8>.

SMANIA FILHO, P. Aplicação de técnicas de programação mista-inteira nãolinear para a programação de produção em refinaria de petróleo. $150 \mathrm{p}$. Dissertação (Mestrado) — Escola Politécnica da USP, São Paulo, jul 2002.

TRANSPETRO S.A. Dutos de Transporte: Capacidades, Preferência do Proprietário e Histórico de Transporte. Rio de Janeiro, mar 2002. Seção: Negócios/Dutos e Terminais. Disponível em: < http://www.transpetro.com.br/portugues/negocios/dutosTerminais/files/DutosCapDisp.xls>. Acesso em: 06 maio 2005.

TRANSPETRO S.A. Dutos de Transporte: Descrição das Instalações. Rio de Janeiro, jul 2004. Seção: Negócios/Dutos e Terminais. Disponível em: $<$ http://www.transpetro.com.br/portugues/negocios/dutosTerminais/files/DutosCurtosCapacidades.xls >. Acesso em: 06 maio 2005.

TRANSPETRO S.A. Tancagem Nominal $\left(\mathrm{m}^{3}\right)$ em Terminais Aquaviários. Rio de Janeiro, jun 2004. Seção: Negócios/Dutos e Terminais. Disponível em: <http://www.transpetro.com.br/portugues/negocios/dutosTerminais/files/TermAq.pdf>. Acesso em: 06 maio 2005. 
UNIVERSIDADE DE SÃO PAULO. ESCOLA POLITÉCNICA. DIVISÃO DE BIBLIOTECA. Diretrizes para apresentação de dissertações e teses. São Paulo, 2006. 103 p. Disponível em: <http://www.poli.usp.br/Bibliotecas/PublicacoesOnLine/Diretrizes3.pdf>. Acesso em: 05 fevereiro 2007.

WILLIAMS, H. P. Model Building in Mathematical Programming. 4. ed. New York: John Wiley \& Sons, LTD, 1999. ISBN 0471997889. 